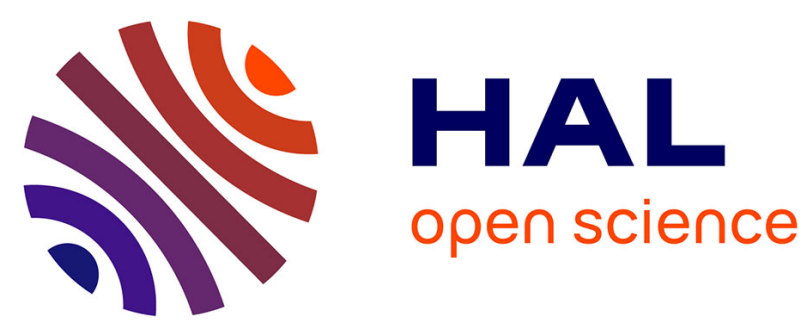

\title{
Les épingles à tête anthropomorphe stylisée: un accessoire de la coiffure féminine de l'Antiquité tardive
} Isabelle Rodet-Belarbi, Paul van Ossel

\section{To cite this version:}

Isabelle Rodet-Belarbi, Paul van Ossel. Les épingles à tête anthropomorphe stylisée: un accessoire de la coiffure féminine de l'Antiquité tardive. Gallia - Archéologie de la France antique, 2003, 60, pp.319-368. 10.3406/galia.2003.3057 . hal-01909342

\section{HAL Id: hal-01909342 \\ https://hal.science/hal-01909342}

Submitted on 9 Jan 2020

HAL is a multi-disciplinary open access archive for the deposit and dissemination of scientific research documents, whether they are published or not. The documents may come from teaching and research institutions in France or abroad, or from public or private research centers.
L'archive ouverte pluridisciplinaire HAL, est destinée au dépôt et à la diffusion de documents scientifiques de niveau recherche, publiés ou non, émanant des établissements d'enseignement et de recherche français ou étrangers, des laboratoires publics ou privés.

\section{(ㅇ)(1) $\$$}

Distributed under a Creative Commons Attribution - NonCommercial - NoDerivatives $\mid 4.0$ 


\title{
LES ÉPINGLES À TÊTE
}

\section{ANTHROPOMORPHE STYLISÉE}

\section{Un accessoire de la coiffure féminine de l'Antiquité tardive}

\author{
Isabelle RodET-BELARBI* et Paul VAN OSSEL ${ }^{* *}$
}

\begin{abstract}
Mots-clés. Épingle en os, Saint-Denis, Paris, Escolives-Sainte-Camille, Gaule, Bretagne, Germanies, Antiquité tardive, coiffure féminine, mode.

Résumé. La découverte à Saint-Denis de déchets de taille, d'ébauches et d'objets finis permet de comprendre la chaîne de fabrication

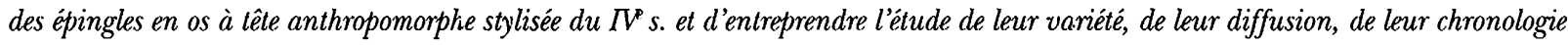
et de leur utilisation. En dépit de sa modestie, cet accessoire de la coiffure féminine se révèle être un témoin précieux pour appréhender les alliludes culturelles de certaines couches de la population provinciale gallo-romaine et pour juger de l'enracinement de la civilisation antique dans les provinces septentrionales de l'Empire romain.
\end{abstract}

Key-words. Hairpin, Saint-Denis, Paris, Escolives-Sainte-Camille, Gaul, Britannia, Germany, Late Antiquity, womens'head-dress, fashion.

Abstract. The discovery in Saint-Denis of waste material, rough-outs and finished pieces has provided an understanding of the manufacturing process of $4^{\text {th }}$ century bone pins with stylized anthropomorphic heads and has enabled a sludy of their variely, distribution, chronology and use. This otherwise modest accessory of womens'head-dress provided precious insight into cultural attitudes amongst certain layers of the provincial Gallo-Roman population and the extent to wich the civilisation of Antiquity took root in the northern provinces of the Roman Empire.

Translated by David COXALL.

Schlagwörter. Haarnadel, Saint-Denis, Paris, Escolives-Sainte-Camille, Gallien, Britannien, Germanien, Spätantike, Haartracht, Mode.

Zusammenfassung. In Saint-Denis bei Paris sind Abfälle, Halbfabrikate und Endprodukte entdeckt worden die es uns erlauben die Herstellungstechnik der Nadeln aus Bein mil stilisiertem anthropomorphen Kopf aus dem 4. Jahrhundert nachzuvollziehen und eine Studie nach ihrer Vielfaltigkeit, Verbreilung, Chronologie und Gebrauch zu unternehmen. Wenn auch sehr bescheiden, hat dieses Zubehörr der weiblichen Haartracht sich als ein wichtiges Zeugnis gezeigt um die kulturellen Gewohnheiten bestimmter Schichten der gallorömischen Bevölkerung zu verstehen und um einzuschätzen in wie fern sich die Kultur der Antike in den nördlichen Provinzen des römischen Reichs behauptet hat.

Übersetzt vom Wim DIJKMAN

Les épingles en os sont des objets domestiques communs dans la culture matérielle de l'Antiquité. Parmi les nombreuses séries reconnues, celles dont le sommet représente une tête humaine fortement stylisée forment un groupe homogène, facilement reconnaissable et bien représenté dans le nord et l'est de la Gaule, ainsi que dans les

* Arçéóöoologue à l'INRAP, chercheur associé à l'UMR 6130 du CNRS, Centre d'études Préhistoire, Antiquité, Moyen Âge, 250 rue Albert-Einstein, Sophia Antipolis, F-06560 Valbonnc.

** UMR 7041 du CNRS, Maison de l'archéologie et de l'ethnologie, 21 allée de l'Université, F-92023 Nanterre Cedex. 
Germanies et en Bretagne. Ces épingles sont caractérisées par une facture extrêmement simplifiée. La gravure des tempes, du front, des arcades sourcilières, du nez. et de la bouche est à peine esquissée par des méplats sommaires et des incisions plus ou moins profondes. L'arrière de la tête est un simple pan coupé en biais sur lequel la chevelure est représentée uniquement par quelques incisions disposées verticalement ou en biais. Sauf exception, les yeux ne sont jamais détaillés (fig. $1 \mathrm{~A}$ ).

Ces artéfacts se rattachent à la famille des épingles à tête anthropomorphe, dont les plus connues représentent des bustes féminins figuratifs, et qui sont attestées à partir du $\mathrm{I}^{\mathrm{er}} \mathrm{s}$. de notre ère jusqu'à la fin du IV $\mathrm{V}^{\mathrm{e}} \mathrm{s}$. au moins. Ils s'en différencient par la forte stylisation de la figuration humaine qui les éloigne des canons plus classiques de l'art antique. En ce sens, ils sont immédiatement identifiables.

Leur caractère typé en fait des témoins privilégiés pour analyser les procédés de fabrication, le contexte dans lequel ils ont été utilisés, ainsi que la période durant laquelle ils ont été en vogue. L'apparente cohérence du schéma iconographique a pu laisser croire à une fabrication standardisée, peut-être limitée à un petit nombre d'ateliers (Béal, 1996, p. 17) ou même à un atelier commun (Ferdière, 1979, p. 43) ' . Toutes ces épingles se ressemblent en effet fortement et ne diffèrent à première vue que par des détails. La question de l'unité du modèle ou, du moins, de son traitement est dès lors posée. Apporter une réponse implique d'entreprendre l'étude de ces objets, d'en dresser l'inventaire - aussi complet que possible -, de déceler les procédés de fabrication, d'en analyser les similitudes et les différences. La démarche conduit à aborder la définition du type et de ses éventuels sous-types, à cerner leur origine et leurs développements successifs. Il s'agit ensuite de les replacer dans leur contexte et d'établir leur originalité parmi de nombreuses autres séries d'objets similaires. Il s'agit enfin de répondre à la question de leur diffusion, localisée dans un espace restreint de l'Empire romain.

Depuis plusieurs années, des chercheurs s'intéressent à ces épingles ${ }^{2}$. Des inventaires de découvertes ont été publiès dans des catalogues de musées. Tant que la perspective se limitait à nourrir une liste de trouvailles et la carte de leur diffusion, l'étude n'a pas vraiment progressé. En établissant

1. Dans la réédition de cette étude (Ferdière dir., 1983, p. 1 : addenda et corrigenda), l'auteur revient sur cette hypothèse et penche plutôt pour une convergence culturelle.

2. Vers le milieu des années 1980 , un article collectif consacré à ce type d'épingles et regroupant Jean-Claude Béal, Luc Bourgeois, Michel Feugère et Paul Van Ossel avait ćté projeté, mais n'avait pu aboutir. La présente étude a bénéficié des notes manuscrites réunies à cette ćpoquc par les différents collaborateurs, qui nous les ont gracieusement remises.
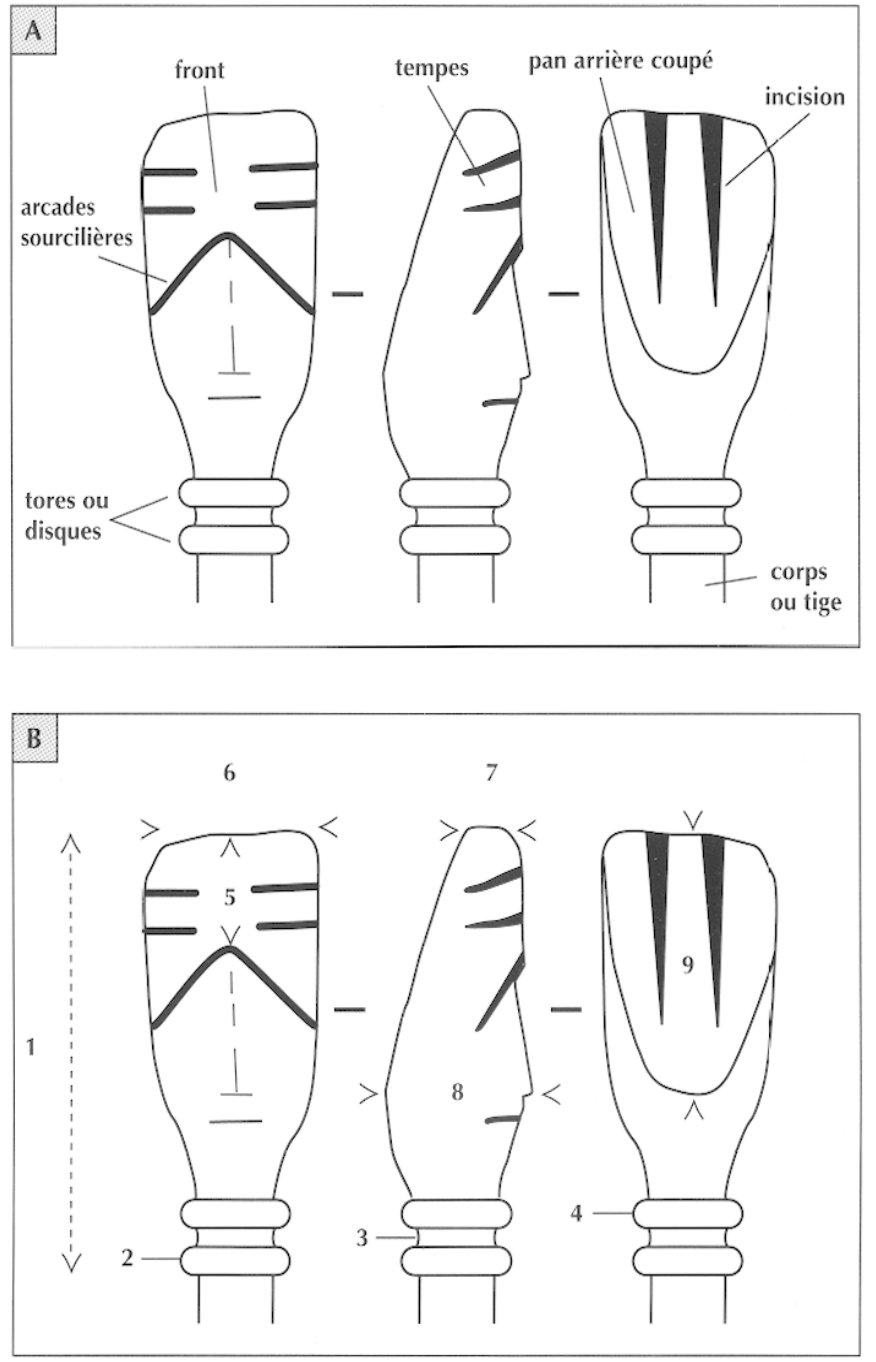

Fig. 1 - Schéma théorique d'une épingle à tête anthropomorphe stylisée. A, appellations usuelles; $B$, localisation des coles de mesures (voir tableaux) : 1, hauteur du sommet de la tête au deuxième tore; 2, hauteur du deuxième tore ; 3, hauteur de la gorge; 4, hauteur du premier tore; 5 , hauteur entre la pointe du cheuron marquant les arcades sourcilières et le sommet de la tête; 6 , largeur maximale de la tête; 7, épaisseur de la tête à son sommet; 8, épaisseur de la tête à la hauteur du nez et de la bouche; 9, hauteur du pan coupé (DAO P. Van Ossel, CNRS).

un lien incontestable entre une activité de taille et la fabrication de ces épingles bien particulières, la découverte à Saint-Denis (Seine-Saint-Denis) d'un petit lot d'ébauches à différents stades de finition donne à l'analyse un nouveau départ. L'étude de ce lot a conduit à rechercher d'autres sites où des rebuts de fabrication de ce type d'objets ont pu être découverts. Le seul lieu identifié depuis longtemps est la ville de Trier, où a été trouvée une épingle inachevée (voir infra, catalogue, $\mathrm{n}^{\circ} 28$, p. 352). Une fois écartés les signale- 
ments incertains ${ }^{3}$, les ateliers ayant façonné des épingles différentes ou de types indéfinis ${ }^{4}$ et les "soupçons " d'ateliers ${ }^{5}$, deux autres lieux potentiels de fabrication d'épingles à tête anthropomorphe stylisée sont apparus. Le premier est Paris, où un lot de déchets de taille a été découvert lors des fouilles du XIX ${ }^{\mathrm{e}}$ s. à un endroit non localisé (Dureuil, Béal, 1996, p. 41-49). Le second est le site d'Escolives-SainteCamille (Yonne), où un atelier de tabletterie a été publié (Prost, 1983). Les épingles, les ébauches et les rebuts de taille de ces deux sites ont été systématiquement comparés avec ceux de Saint-Denis.

Toutes les ébauches et les épingles ont été observées minutieusement à l'œil nu ou à la loupe. La présence et l'emplacement des traces de travail, le degré de finition de la pièce, les défauts des ébauches et la présence éventuelle de patine ont été notés. Onze mesures ont été prises en divers points (fig. 1 B). Elles permettent de replacer les ébauches sur une grille de dimensions et aident à préciser la raison de leur non-achèvement. L'objectif était de déterminer les éventuelles variantes, de manière à pouvoir les regrouper ensuite par lieu de découverte et par contexte chronologique.

\section{LES ÉPINGLES EN OS ET LES REBUTS DE FABRICATION DE SAINT-DENIS}

\section{CONTEXTE DE DÉCOUVERTE}

Les épingles et rebuts de fabrication d'épingles en os de Saint-Denis proviennent quasiment tous d'un même contexte(fig. 2) : le remplissage d'un fossé à profil arrondi, fouillé en 1988 au nord de la basilique (A). Une épingle de type anthropomorphe a été découverte aussi dans le radier d'une voie (2702) fouillée sous l'église SaintPierre (D).

3. Par exemple Biehler, Schlemaire, 1974 el Guillaumc, 1970, p. 75.

4. Voir Christophe, Ertlé, 1969-1970, p. 22-23 ; Billoret, 1974, p. 356 ; Santrot et al., 1975, p. 120-121 ; Bourgeois, Tuffreau-Libre, 1981, p. 115 ; Biton, 1985 ; Pilet, 1986 ; Rodet-Belarbi, 1990a et 1990b ; Cribellier, 1993, p. 75-76 ; Dureuil, Béal, 1996, p. 15 ; Deschler-Erb, 1998.

5. L'épingle de Maastricht ne présente ni tore ni incision sur le pan coupé et sur le front (voir infra, catalogue, $\mathrm{n}^{\circ} 291$, p. 362). Unc des ćpingles de Châteaubleau porte aussi un pan coupé lisse ct des tores à peine ébauchés (voir infra, catalogue $n^{\circ} 128$, p. 356). Ces deux pièces sont peut-être inachevées. Beaucoup plus sûres sont les deux ébauches à des stades de finition différents provenant de l'archevêché de Sens (voir infra, catalogue, $\mathrm{n}^{\text {os }} 247$ et 248 , p. 360, et fig. 10, $\mathrm{n}^{\text {os }} 20-21$ ), mais l'absence de toute indication sur la présence de déchets de fabrication d'épingles sur cetté fouille empêche malheurcusement de conclurc à la présence d'un atelier sur place.
Ce fossé, identifié sous le $\mathrm{n}^{\circ} 976$, a été fouillé en trois tronçons sur une distance de $43 \mathrm{~m}$. Orienté est-ouest, il se trouve à environ $80 \mathrm{~m}$ au nord-est de la memoria du BasEmpire (B), non loin d'un bassin en mortier (C) dont la datation n'est pas établie avec précision (Bas-Empire ou époque mérovingienne?). Topographiquement, on se trouve sur la partie sommitale - légèrement reportée vers le nord-ouest - de l'étroite élévation de terrain occupée par l'actuelle basilique (fig. 4). Cette petite éminence, qui culmine aujourd'hui à $33 \mathrm{~m}$ NGF environ, forme une avancée entourée sur trois côtés par des dépressions marécageuses au fond desquelles serpentent le ru de Montfort au sud et à l'ouest ainsi que le Rouillon au nord. À l'emplacement du fossé, la topographie ancienn ₹ présente une très faible pente vers le nord, vers le Rouill $n$, qui coule à l'altitude de $27 \mathrm{~m} \mathrm{NGF}$, soit un dénivelé de $5 \mathrm{~m}$ à peine. Le fossé 976 recoupe en écharpe l'extrémité du platĩau.

Cé fossé est largement perturbé par des structures postérieures, d'où un tracé morcelé et une conservation inégale de son profil qui présente une inclinaison moyenne de $58^{\circ}$. Sa largeur maximale observée est de $1,80 \mathrm{~m}$; elle ne devait guère dépasser $2 \mathrm{~m}$ à son ouverture au niveau du sol antique (31 m NGF). Sa profondeur diminue d'ouest en est: environ $1,10 \mathrm{~m}$ à l'ouest, $0,90 \mathrm{~m}$ au centre et $0,70 \mathrm{~m}$ à l'extrémité orientale. Le fond présente un léger surcreusement qui permet de suivre aisément son axe médian. Son comblemcnt varic selon les tronçons et parties de tronçons. Toutefois, il se dégage de la lecture des fiches d'enregistrement une impression générale d'homogénéité, malgré quelques différences locales. Les remblais sont généralement sableux et compacts, sauf en un endroit où le fond du fossé est comblé par une couche damée (US 971), composée d'une alternance de fines pellicules de cendres (tabl. I). Les couches supérieures du comblement, quand elles sont conscrvées (uniquement tronçon occidental), sont plus hétérogènes et contiennent des cailloux (US 709) ou des fragments de charbon de bois (US 1113, 969).

Les trois tronçons du fossé, appelés occidental, médian et oriental, ont fait l'objet d'une fouille et d'un enregistrement séparés (fig. 3 et tabl. I).

- Le tronçon occidental, le plus long $(12,40 \mathrm{~m})$ et le mieux conservé, est subdivisé en trois parties : A, B et C. Le comblement des parties A et B est constitué des US 718 et 719 , recouvertes localement par l'US 709. Celui de la partie C comprend les US 971, 970, 969 et 1113. Toutes ces couches ont livré des épingles ou des rebuts de fabrication. Deux coupes présentent le profil du fossé dans ce secteur (fig. 2 et 3 , coupes 1 et 2 ). Le fossé est scellé par un fond de cabane d'époque plus récente (US 727). Son remplissage (US 710) a livré quelques fragments de rebuts de fabrication qui appartiennent vraisemblablement à la série trouvée dans 


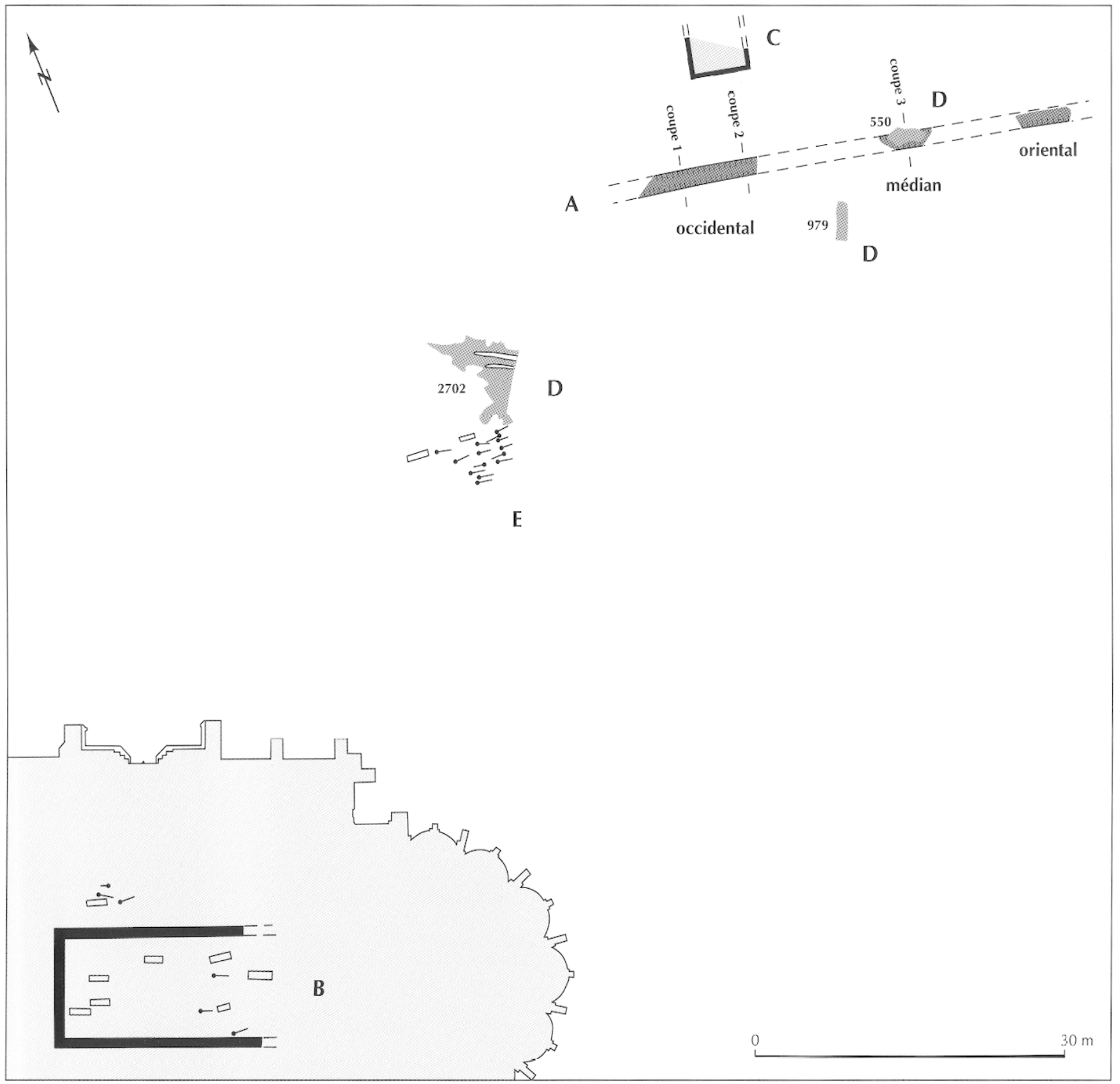

Fig. 2 - Plan des vestiges du Bas-Empire à Saint-Denis avec localisation sommaire du fossé 976 (état 2001) : A, fossé 976 ; B, basilique et memoria de Sainte-Geneviève ; $C$, bassin; $D$, tronçons de voie; $E$, tombes du Bas-Empire et de l'époque mérovingienne (plan M. Wyss, unité archéologique de Saint-Denis ; DAO P. Van Ossel, CNRS).

le fossé, ainsi que des fragments de céramique qui recollent avec certains autres trouvés dans l'US 719. La présence de ce matériel très semblable confirme une perturbation du fossé pendant le haut Moyen Âge (voir infra, p. 328).

- Le tronçon médian, suivi sur environ $5 \mathrm{~m}$, présente un intérêt tout particulier en raison de son recouvrement par un empierrement (US 550) appartenant à un chemin de
l'Antiquité tardive ${ }^{6}$ qui est présumé traverser tout le site pour se raccorder à la grande voie romaine (l'Estrée) vers Rouen (fig. $2 \mathrm{D}$ et fig. 4). La fonction structurante de cette

6. Cette voie est constituéc par la réunion de trois tronçons disjoints (US 2702, 979 et 550) qui présentent des caractéristiques communes (empierrement peu épais posé directement sur le sol naturel, mobilier 


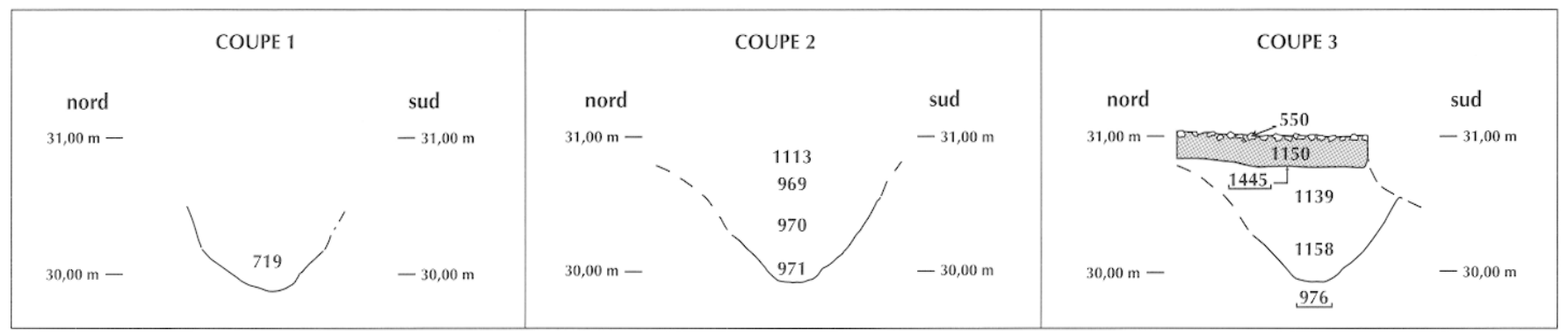

Fig. 3 - Coupes du fossé 976 de Saint-Denis (DAO P. Van Ossel, CNRS).

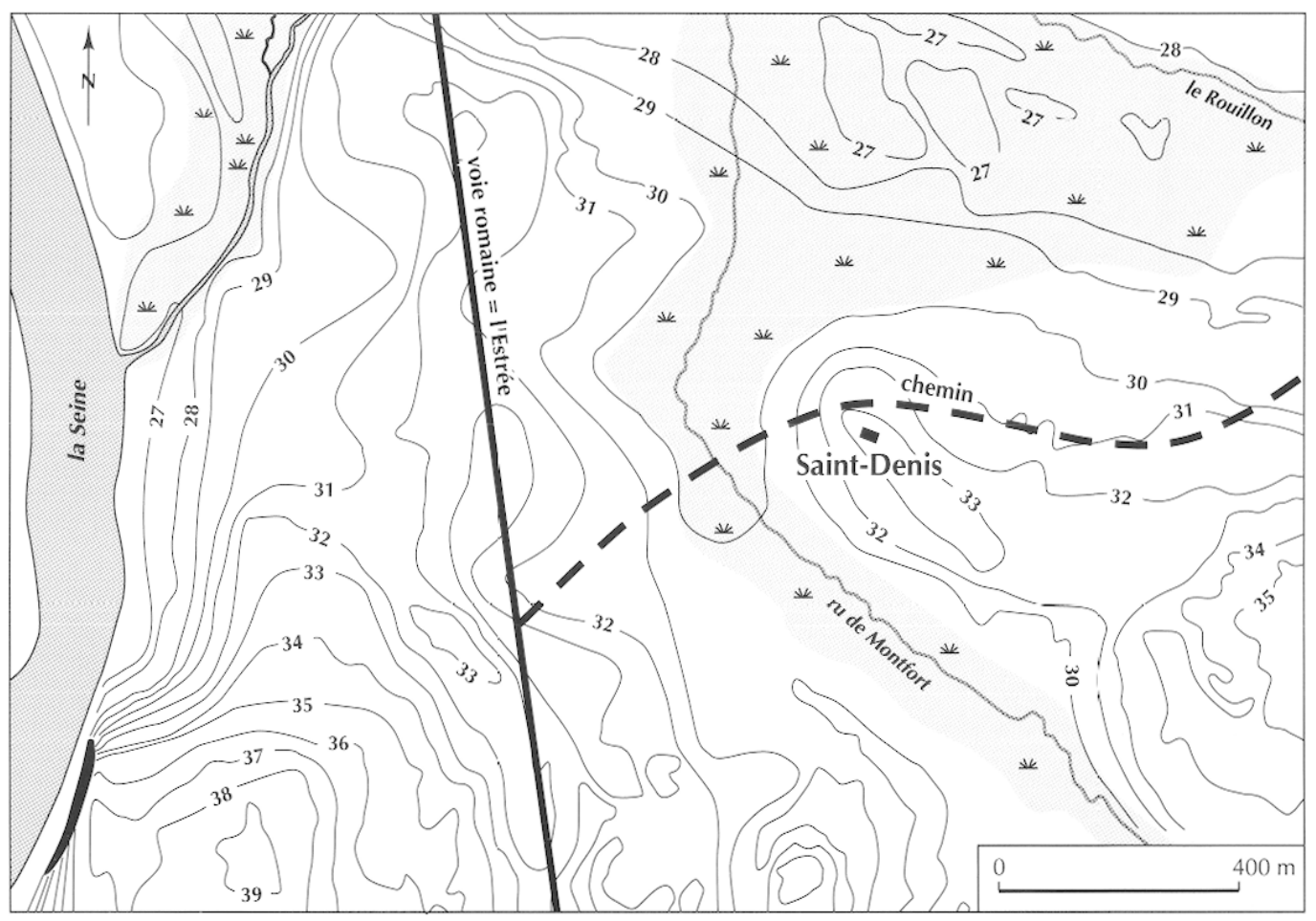

Fig. 4 - Plan de situation topographique de Saint-Denis (d'après Wyss dir., 1996, p. 187, fig. 171 ; DAO P. Van Ossel, CNRS).

voie dans la topographie tardo-antique et mérovingienne du site a été soulignée (Wyss dir., 1996, p. 28-29 et 187-188) en raison des sépultures établies le long de son tracé (fig. $2 \mathrm{E}$ ). Une coupe présente le profil du fossé et son recouvrement par la voie constituée par un remblai et un mince radier de cailloux (fig. 2 et fig. 3, coupe 3). Le comblement du fossé est homogène et comprend deux couches (US 1139 et 1158), dont seule la seconde, celle du fond, a livré des rebuts de fabrication.

- Le tronçon oriental, le moins bien conservé, a été suivi sur environ $5 \mathrm{~m}$. Seul son fond a été observé. Son

de la fin du IVe s.-début du Ve s.), mais qui restent en réalité difficiles à relier en raison de l'orientation divergente des ornières observées à leur surface. comblement (US 1159) a, lui aussi, livré des rebuts de fabrication.

\section{MOBILIER ET DATATION}

Le mobilier recueilli dans les couches du comblement primaire du fossé (US 718, 719, 971, 1158 et 1159) appartient globalement à la seconde moitié du IV ${ }^{\mathrm{e}} \mathrm{s}$.

Les trente-huit monnaies en bronze mises au jour constituent un lot assez important dans ce type de contexte ${ }^{7}$. Elles comprennent surtout une très forte proportion de pièces des années 350-358 (près de $50 \%$, ce qui est inhabituel dans

7. Les monnaies ont été identifiées par M. Amandry (Cabinet des Médailles). 
Tabl. I - Tableau stratigraphique schématique du fossé 976 de Saint-Denis :

1, rebuts de fabrication; 2, épingles à tête anthropomorphe stylisée ; 3, épingles d'un type différent ; 4, monnaies (avec indication des dates extrêmes).

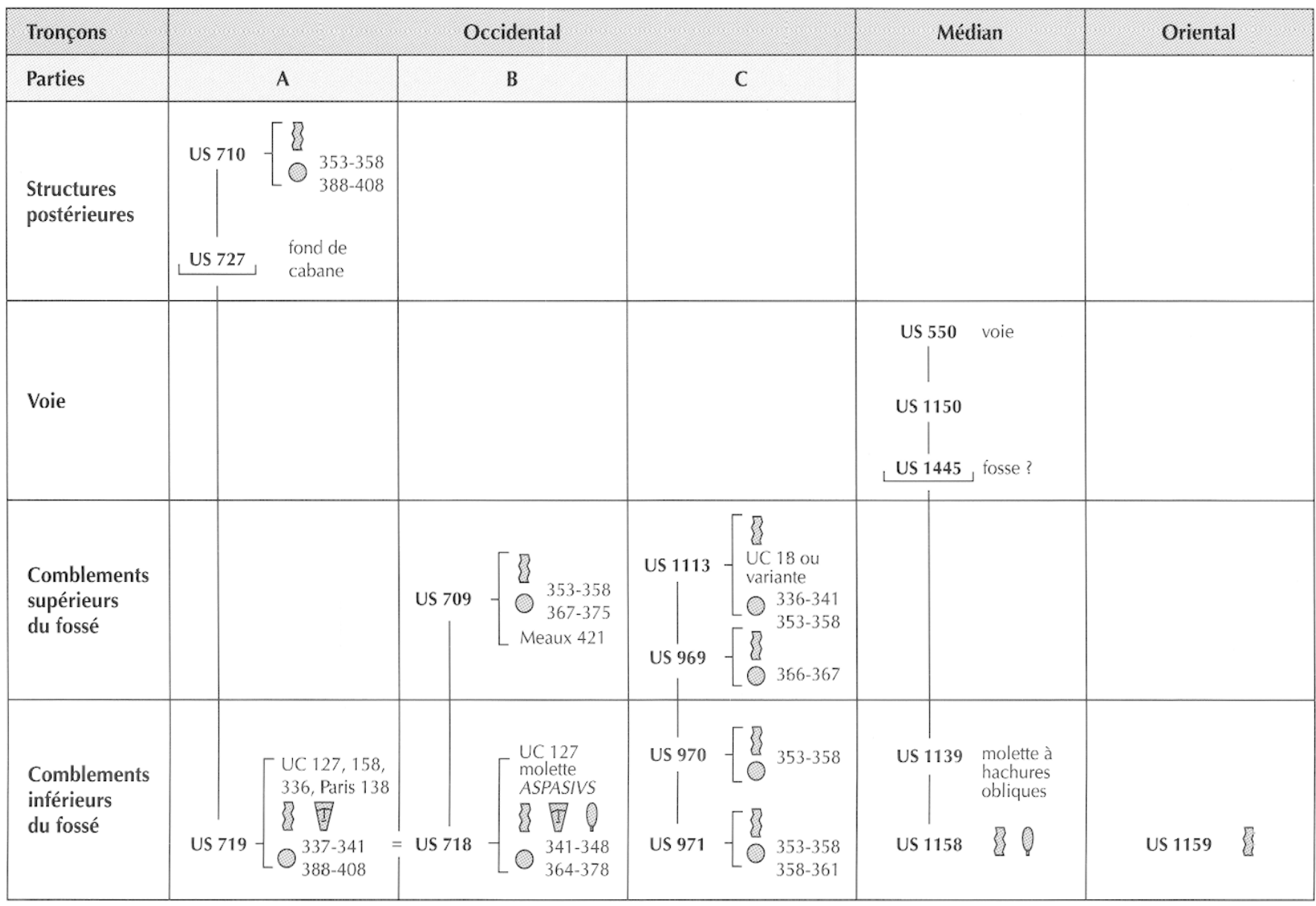

\& 1 目2 $\quad 0_{3} \quad 0_{4}$

Tabl. II - Saint-Denis, fossé 976. Répartition des monnaies des couches de comblement du fossé par période d'émission monétaire (d'après Ravetz, 1964, complété).

\begin{tabular}{|l|c|}
\hline $294-317$ & - \\
\hline $317-330$ & - \\
\hline $330-341$ & 2 \\
\hline $341-348$ & 2 \\
\hline $348-364$ & 17 \\
\hline $364-378$ & 8 \\
\hline $378-388$ & - \\
\hline $388-408$ & 2 \\
\hline indéterminés & 6 \\
\hline Total & 37 \\
\hline
\end{tabular}

la circulation monétaire de l'époque) et des pièces d'époque valentinienne (tabl. II). Aucune monnaie n'est antérieure à $337-341$ et la couche US 971 , constituée de rejets de foyers, ne contient que des monnaies frappées entre 353 et 361 . Seul le comblement de l'US 719 a livré deux monnaies de la dernière période d'émission monétaire (388-408) sans que l'on puisse préciser davantage les émissions. On remarquera aussi que les monnaies provenant de la partie C du tronçon occidental (US 1113, 969, 970, 971) sont globalement plus anciennes et présentent une composition plus homogène (centrée sur le troisième quart du IV ${ }^{e}$ s.) que celles des deux autres parties de ce tronçon.

Les céramiques constituent un ensemble assez caractéristique du dernier tiers ou du dernier quart du IV $\mathbf{s}$. (tabl. III). La terre sigillée totalise près de la moitié des formes identifiées $(49,5 \%)$. Le reste se partage entre la céramique gra- 
Tabl. III - Saint-Denis, fossé 976. Tableau de comptage de la céramique provenant du comblement inférieur du fossé (US 719, 718, 970, 971, 1139, 1158 et 1159) :JVV, Jaulges -Villiers-Vineux ; Ch., Chenet ; a, anse ; $b$, bord; $d$, décor ; f, fond; $p$, paroi.

\begin{tabular}{|c|c|c|c|c|c|c|c|c|c|}
\hline Catégorie & Groupe & Sous-groupe & NR & $\%$ NR cat. & Forme & Doc. & NMI & $\%$ NMI Total & Figure 5 \\
\hline \multirow[t]{8}{*}{ dérivé sigillée } & Argonne & Argonne & 14 & $18 \%$ & Ch. 320 & $13 \mathrm{~d}, 1 \mathrm{~b}$ & 13 & $21 \%$ & $n^{0} 1$ \\
\hline & & & 7 & $9 \%$ & Ch. 324 & $7 b$ & 7 & $11 \%$ & \\
\hline & & & 2 & $2 \%$ & Ch. 348 & $1 b, 1 p$ & 1 & $1,5 \%$ & $\mathrm{n}^{0} 6$ \\
\hline & & & 6 & $7,5 \%$ & Ch. $328 / 30$ & $2 p, 4 b$ & 2 & $4 \%$ & $n^{\circ \text { os }} 3-5$ \\
\hline & & & 1 & $1 \%$ & Ch. 304 & $1 b$ & 1 & $1,5 \%$ & $n^{\circ} 2$ \\
\hline & & & 14 & $18 \%$ & gobelet indéterminé & $14 p$ & 1 & $1,5 \%$ & \\
\hline & & & 34 & $42,5 \%$ & indéterminée & $3 f, 31 p$ & 3 & $6 \%$ & \\
\hline & JVV & & 2 & $2 \%$ & Ch. 323 & $1 b, 1 f$ & 2 & $3 \%$ & \\
\hline Total & & & 80 & $100 \%$ & & & & $49,5 \%$ & \\
\hline Céramique fine & engobée & trévire & 1 & & gobelet & $1 \mathrm{p}$ & 1 & $1,5 \%$ & \\
\hline Total & & & 1 & & & & & $1,5 \%$ & \\
\hline \multirow[t]{6}{*}{ commune } & sableuse & grise ou orange & 1 & $2 \%$ & faisselle & $1 \mathrm{~b}$ & 1 & $1,5 \%$ & $n^{\circ} 9$ \\
\hline & & & 13 & $22 \%$ & vase à bord triang. & $3 b, 10 p$ & 2 & $3 \%$ & $n^{\circ 5} 7-8$ \\
\hline & & & 38 & $64 \%$ & indéterminée & $2 b, 34 p, 2 f$ & 4 & $6,5 \%$ & \\
\hline & & & 2 & $3 \%$ & imitation gobelet & $1 b, 1 f$ & 2 & $3 \%$ & \\
\hline & craquelée & claire & 1 & $2 \%$ & indéterminée & $1 p$ & & & \\
\hline & fine & fine lustrée & 4 & $7 \%$ & indéterminée & $4 p$ & & & \\
\hline Total & & & 59 & $100 \%$ & & & & $14 \%$ & \\
\hline \multirow[t]{6}{*}{ granuleuse } & régionale & & 7 & $4 \%$ & jatte Petit IIIB & $7 \mathrm{~b}$ & 5 & $8 \%$ & $\mathrm{n}^{05} 10-18$ \\
\hline & & & 1 & $0,5 \%$ & jatte var. Petit III & $1 \mathrm{~b}$ & 1 & $1,5 \%$ & $\mathrm{n}^{\circ} 19$ \\
\hline & & & 16 & $9 \%$ & vase Alzei 27 & $5 b, 11 p$ & 5 & $8 \%$ & $\mathrm{n}^{\text {os }} 20-23$ \\
\hline & & & 5 & $3 \%$ & cruche tréflée & $1 \mathrm{~b}, 1 \mathrm{a}, 3 \mathrm{p}$ & 2 & $3 \%$ & \\
\hline & & & 4 & $2,5 \%$ & pichet Alzei 30 & $2 \mathrm{~b}, 1 \mathrm{a}, 1 \mathrm{p}$ & 3 & $4,5 \%$ & $\mathrm{n}^{\circ} 24$ \\
\hline & & & 138 & $81 \%$ & indéterminée & $8 f, 130 p$ & 6 & $10 \%$ & \\
\hline Total & & & 171 & $100 \%$ & & & & $35 \%$ & \\
\hline \multicolumn{3}{|l|}{ Total général } & 311 & & & & 62 & $100 \%$ & \\
\hline
\end{tabular}

nuleuse régionale (35\%), une quantité beaucoup plus faible de céramiques communes sableuses $(14 \%)$ et un unique fragment de gobelet trévire en céramique fine. De telles proportions sont représentatives de l'époque. Elles deviennent fréquentes à partir de l'époque valentinienne, pendant laquelle la part de céramique sableuse tend à diminuer progressivement au profit des céramiques sigillées et des céramiques granuleuses.

La sigillée, composée presque exclusivement de récipients issus des productions d'Argonne, illustre un répertoire classique de formes (fig. $5, n^{\text {os }} 1-6$ ). Les molettes sur sigillée comprennent plusieurs décors à petits casiers apparentés à UC 158 et UC 304 (Hübener, 1968, groupe 2), fréquents durant toute la seconde moitié du $\mathrm{IV}^{\mathrm{e}} \mathrm{s}$. (fig. 6 , $\mathrm{n}^{\circ} 1$ ), ainsi que les molettes UC 127 (fig. 6, $\mathrm{n}^{\text {os }} 2-3$ ), UC 336 (fig. $6, n^{\circ} 4$ ), UC 18 ou apparentée (fig. $6, n^{\circ} 5$ ), Meaux 421
(Collectif, 1984a, p. 144, $\mathrm{n}^{\circ}$ 421) (fig. 6, $\mathrm{n}^{\circ} 8$ ), Paris 138 (Van Ossel, 1994, p. 83, $\mathrm{n}^{\circ} 138$ ) (fig. 6, $\mathrm{n}^{\circ} 6$ ) et la molette épigraphique ASPASIVS (fig. $6, \mathrm{n}^{\circ} 7$ ). À l'exception de cette dernière, connue en deux exemplaires seulement à ce jour, ces décors se trouvent souvent associés dans des contextes datables de la seconde moitié du IV ${ }^{e}$ s., en particulier dans la région parisienne.

Parmi les productions plus communes, les formes Petit IIIb (fig. 5, $\mathrm{n}^{\text {os }} 10-19$ ) et Alzei 27 (fig. 5, $\mathrm{n}^{\text {os }}$ 20-23) en céramique rugueuse dérivée des productions granuleuses de l'Eifel constituent l'essentiel du répertoire. Les nombreuses variantes de ces deux formes appartiennent vraisemblablement toutes à des productions régionales. La forme Petit IIIb est attestée dans la région parisienne à partir de l'époque valentinienne, époque à laquelle elle prend le relais de la forme Petit IIIa (= Alzei 28), beaucoup moins 


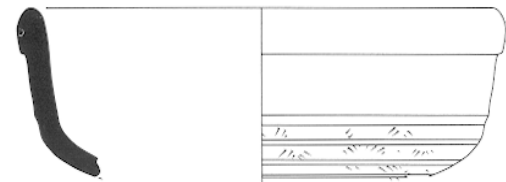

1

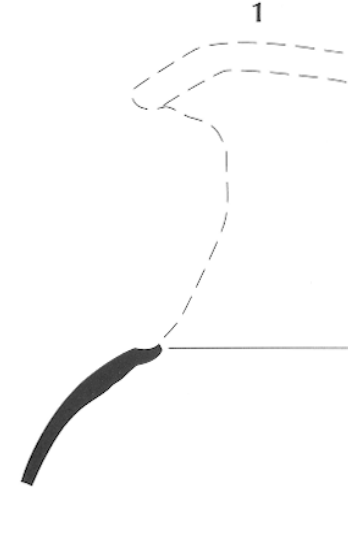

6
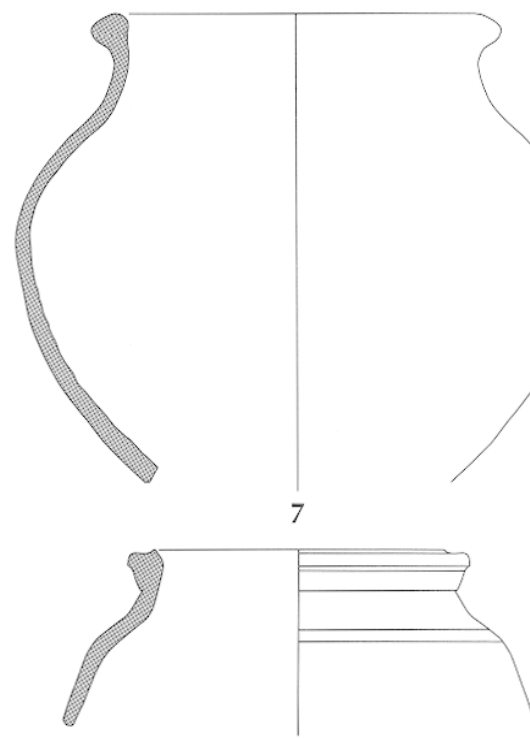

8
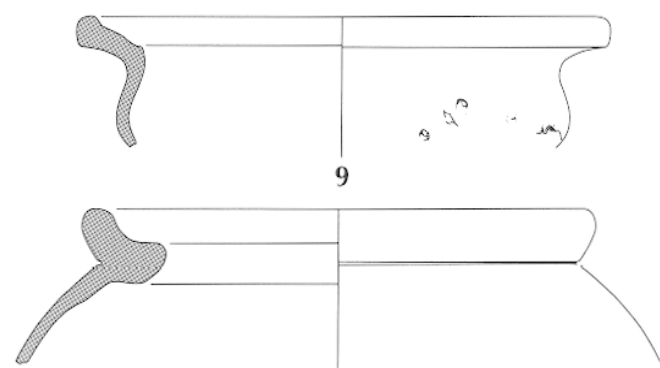

20
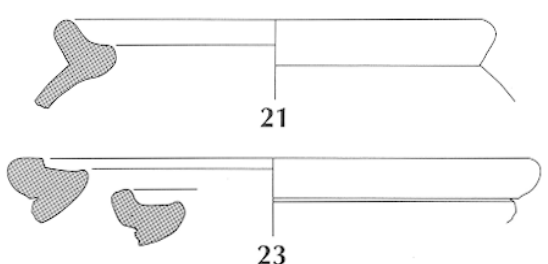

22

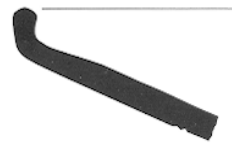

1

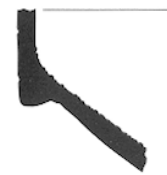

3
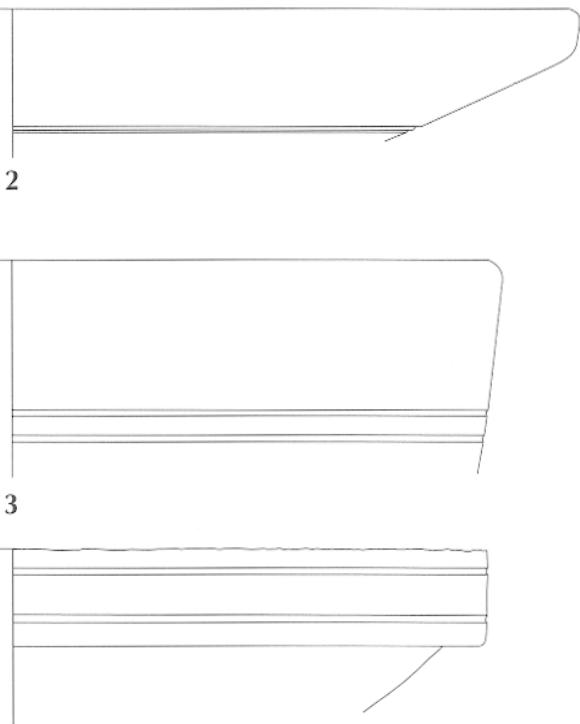

4
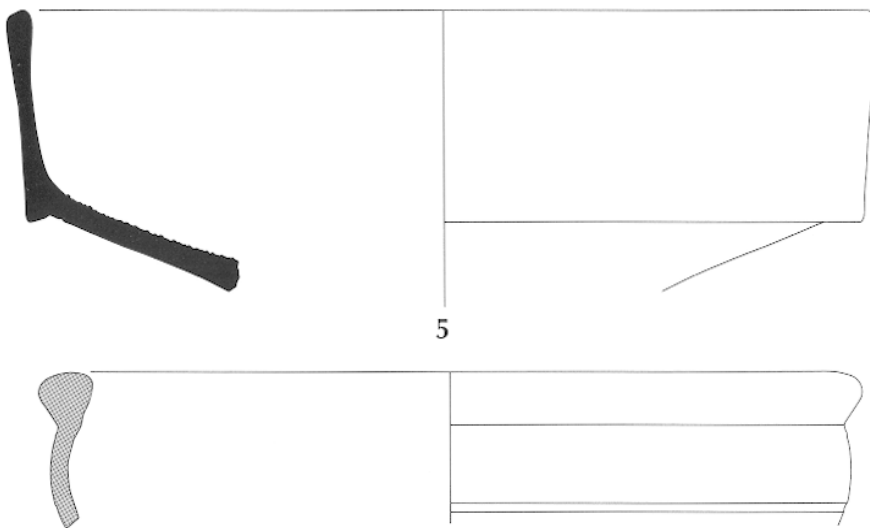

10

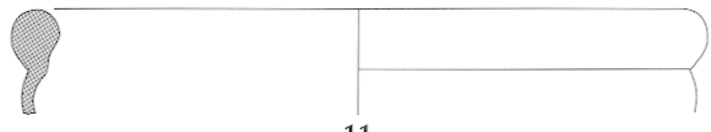

11

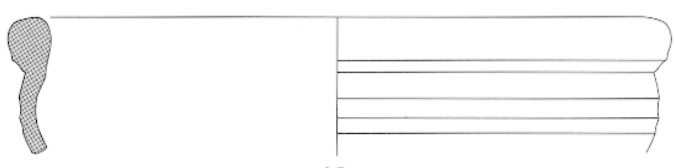

12

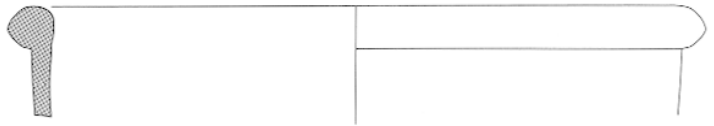

13
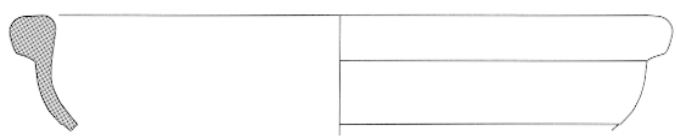

14
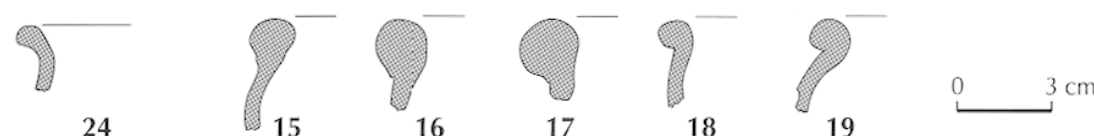

Fig. 5 - Céramique provenant des couches de comblement du fossé 976 de Saint-Denis : coupes noircies, céramique sigillée ; coupes tramées, céramique commune (dessin D. Le Bras, unité archéologique de Saint-Denis ; DAO P. Van Ossel, CNRS). 


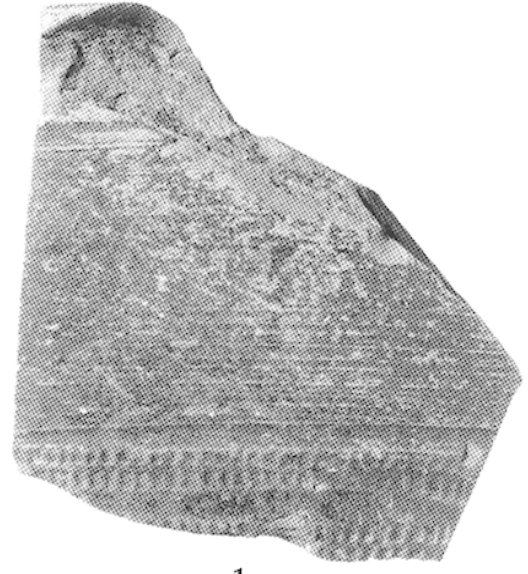

1

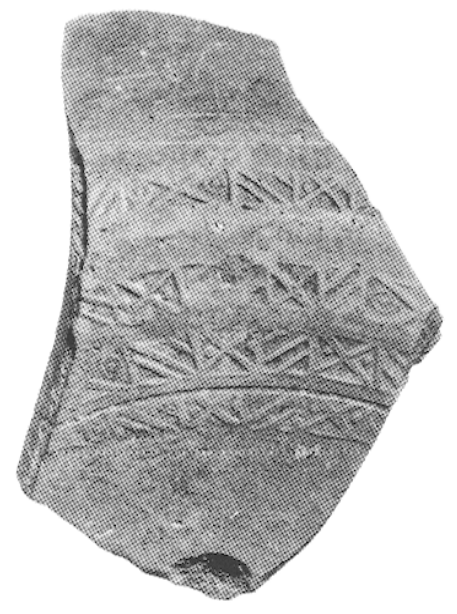

6

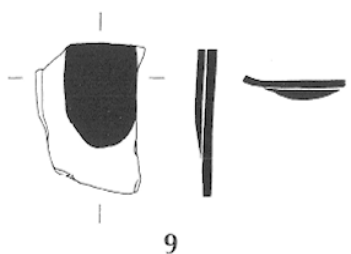

9

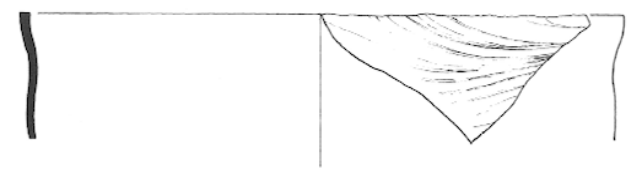

13
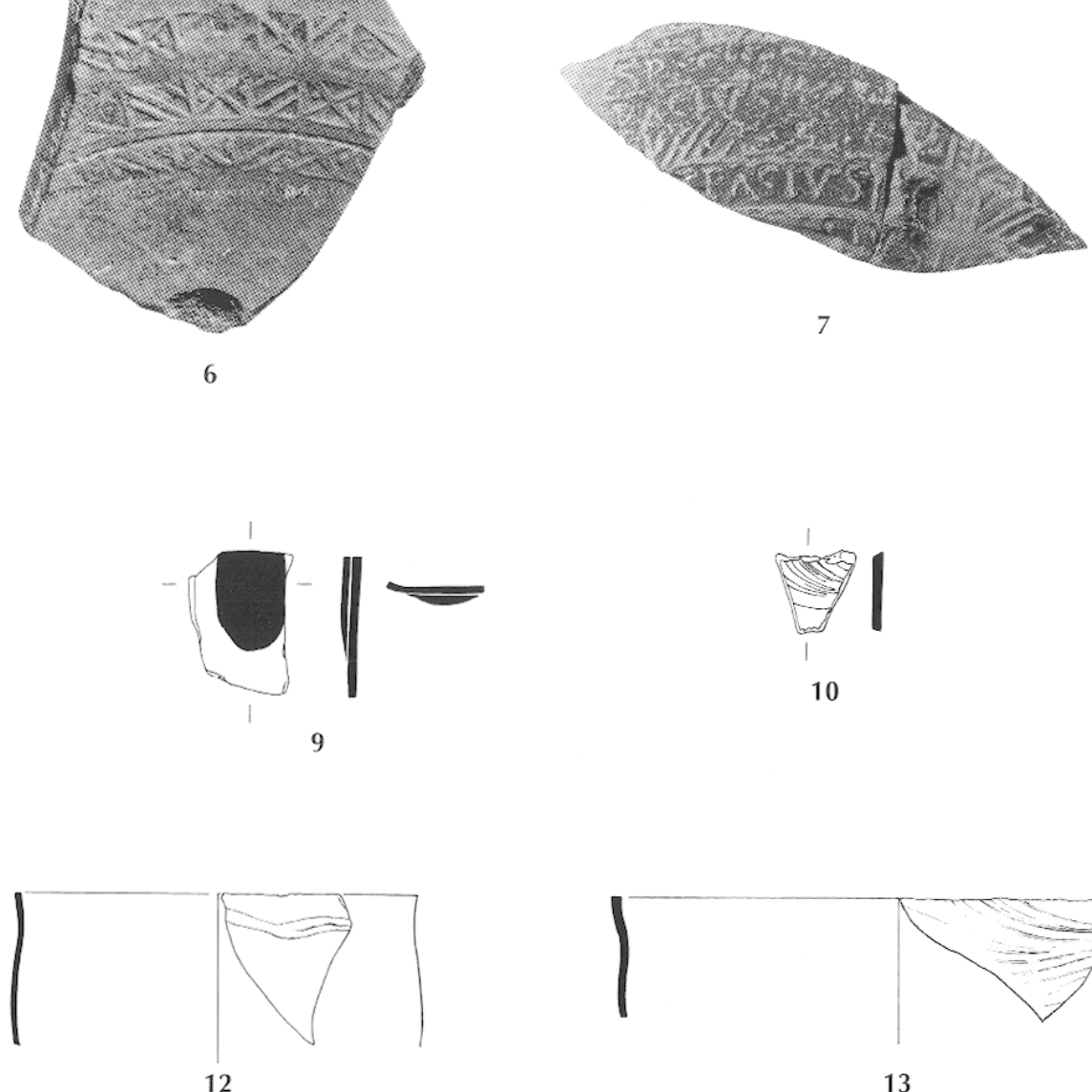
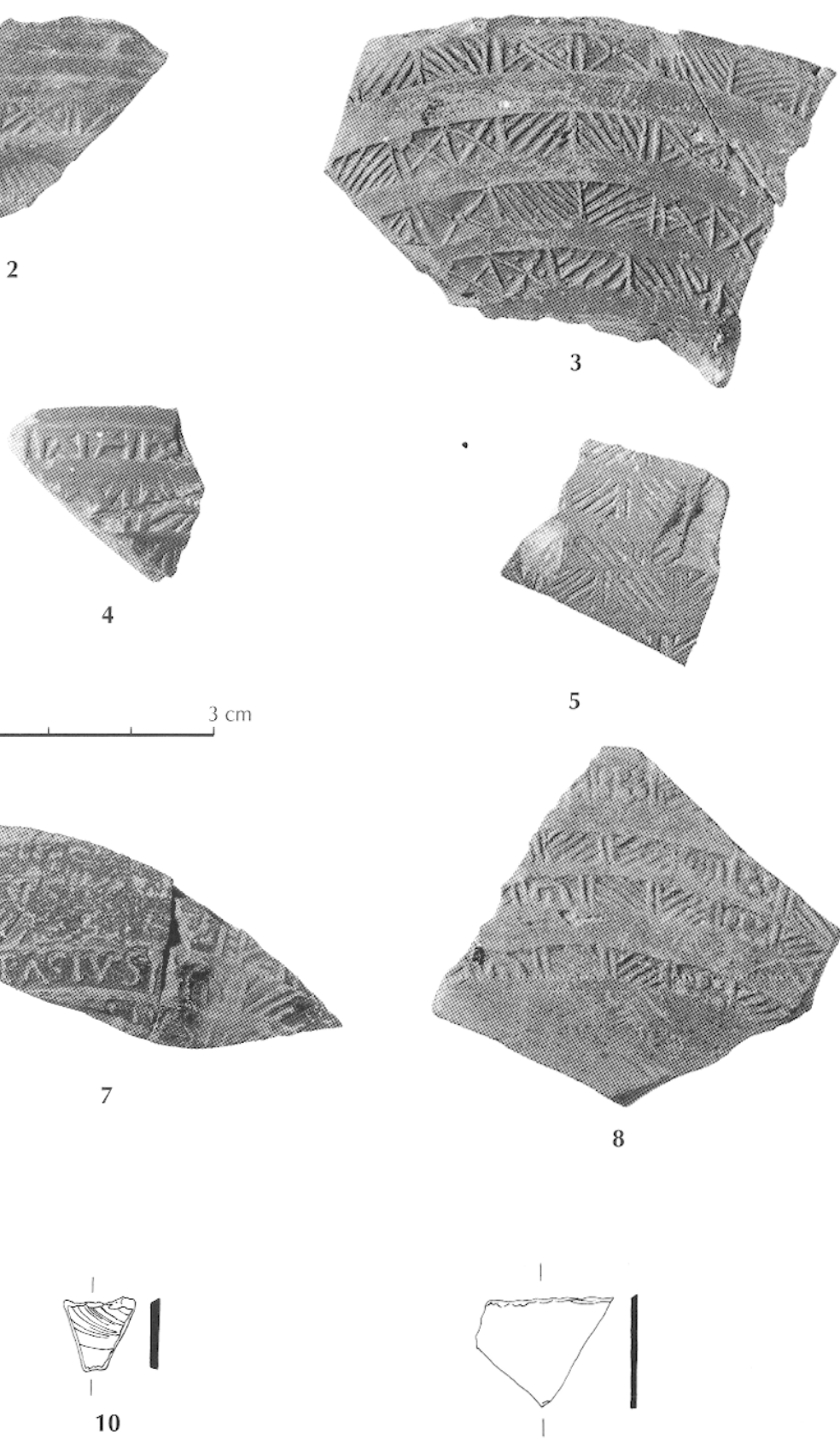

4
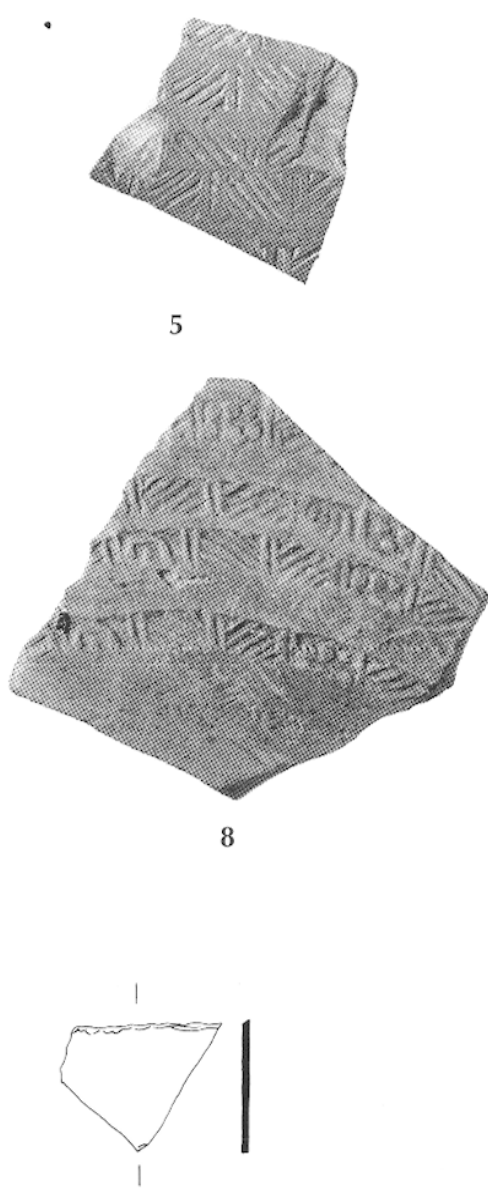

11

7

8

Fig. 6 - Céramique et verre provenanl des couches de comblement du fossé 976 de Saint-Denis : 1-8, molettes sur céramique sigillée d'Argonne; 9-13, verrerie du $N^{*}$ s. (photos M. Esline, CNRS; dessins D. Le Bras, unité archéologique de Saint-Denis). 
répandue (Barat, 1993, p. 175). À Rouen (Seine-Maritime), la forme apparait dans les couches du dernier quart du IVe $s$. de la Cour des Maçons ; elle est plus fréquente à la charnière des $\mathrm{IV}^{\mathrm{c}}-\mathrm{V}^{\mathrm{c}} \mathrm{s}$., sans toutefois dépasser le milieu du $\mathrm{V}^{\mathrm{e}} \mathrm{s} .{ }^{8}$. Cette chronologie a été constatée également en Îlede-France (Séguier dir., 1995, p. 24-25). Les autres formes (fig. 5, nos 7-9 et 24) sont beaucoup plus rares et n'apportent pas de précisions chronologiques supplémentaires.

Parmi les autres mobiliers, on peut noter la présence de quarante-cinq fragments de verrerie, dont deux de verre à vitre, un tesson de gobelet à pastilles en verre bleu (Nuppenglas), semblable à ceux produits dans les ateliers de Köln et de Trier au IV ${ }^{\mathrm{e}}$ s. (Fremersdorf, 1962 ; GoethertPolaschek, 1984, p. 260-261 et $n^{\circ} 139$ ), et plusieurs fragments de gobelets ou bols à bords taillés, très communs au IVes. (fig. 6, nos 9-13).

Des clous de chaussures, des restes de faune, dont des coquilles d'huître, des morceaux de tuile et des restes d'objets en fer et en alliage cuivreux complètent cet ensemble et indiquent sans aucun doute la provenance domestique du matériel rejeté dans le fossé.

Le mobilier du fond de cabane 727 est en revanche nettement plus tardif, malgré de nombreux témoins résiduels arrachés à leur contexte d'origine lors de son aménagement. La céramique mise au jour dans cette structure d'habitat appartient principalement à la seconde moitié du Ves., voire au début du VI ${ }^{\mathrm{e}} \mathrm{s}$. On y trouve en particulier les premières molettes sur céramique mérovingienne, datées par R. Legoux de sa phase A-B-C 1, vers 450/460-475 (Legoux, 1998, p. 137-138). Ce matériel indique un décalage d'environ un siècle entre le comblement du fossé et celui du fond de cabane. On y a trouvé aussi quelques tessons de céramique carolingienne du VIII $^{e}$ s., témoins vraisemblables d'une contamination avec les niveaux postérieurs.

\section{LES ÉPINGLES ET LES REBUTS DE FABRICATION DU FOSSÉ 976}

Le fossé 976 de Saint-Denis a livré uniquement des éléments de fabrication d'épingles, à l'exclusion de tout autre objet. Ces éléments peuvent être classés en cinq catégories distinctes: de gros rejets de matière première (extrémités de métacarpes et de métatarses), des éclats de débitage de diaphyse lors de l'extraction des baguettes, des rebuts de taille des baguettes, des ébauches et, enfin, des

8. Voir Y. Adrian avec la collab. de P. Van Ossel, Rouen (76, SeineMaritime). La Cathédrale, "Cour des Masons " (fouilles programmées de 1989 à 1993 sous la direction de Jacques Le Maho-CNRS, CRAM de Caen). Étude du mobilier du Bas-Empire et du haut Moyen Âge (céramique, verre et petit mobilier), étude manuscrite inédite, Rouen, 1997, 195 p. épingles achevées. Ces dernières comprennent cinq épingles à tête anthropomorphe stylisée, quatre épingles appartenant à d'autres types (avec tête sphérique, en pointe, en pomme de pin) et quatre fragments de corps dont l'appartenance à un type particulier ne peut être déterminée.

Les déchets, les ébauches et les épingles ratées apportent de multiples renseignements sur les techniques de fabrication. Le lot dionysien contient des témoins de toutes les étapes de la chaîne de façonnage. Chacune d'elles peut être reconstituée. Seule leur quantité varie selon la catégorie considérée.

\section{LES DÉCHETS RÉSULTANT DE LA RÉCUPÉRATION DE LA MATIÈRE PREMIÈRE}

La première catégorie regroupe les rejets les plus nombreux, c'est-à-dire les extrémités proximales (ou supérieures) ou distales (ou inférieures) des métapodes (métacarpe ou métatarse) de bovins, jetées après récupération de la diaphyse (ou corps de l'os), utilisée comme réserve de matière premic̀rc. En raison de leur grande résistance aux diverses conditions de conservation, elles sont très communément retrouvées sur les sites gallo-romains où elles représentent un des premiers témoignages du travail de l'os. Sur les trente et un exemplaires, on compte neuf extrémités proximales et treize extrémités distales de métacarpes, six extrémités proximales et trois extrémités distales de métatarses (tabl. IV). Le sciage a été effectué près de la surface articulaire. Aucun canal médullaire n'a été nettoyé et travaillé.

Ces déchets sont très standardisés ; seuls quelques-uns possèdent une particularité. C'est le cas pour deux extrémités proximales de métatarses $(719 / 21$ et $1159 / 1 b)$ qui portent chacune des traces de coups de couperet donnés à plusieurs reprises sur le tubercule au point d'attache des ligaments, sur la surface articulaire et/ou sur la face plantaire. L'extrémité proximale de métacarpe de bovin $1158 / 3$ porte la trace d'un léger coup de couperet sur le bord. L'intention était peut-être de nettoyer l'os au mieux, même rapidement, avant sciage. L'exemplaire $1159 / \mathrm{lc}$ correspond à un déchet issu d'une extrémité distale épiphysée de métatarse droit de bœuf. On remarque sous le plan de coupe une entaille correspondant à un premier essai infructueux de sciage (fig. $7, \mathrm{n}^{\circ} 1$ ).

\section{LES ÉCLATS PROVENANT DU DÉBITAGE DE LA DIAPHYSE LORS DE L'EXTRACTION DES BAGUETTES}

Les quatorze éléments retrouvés possèdent tous au moins une extrémité sciée (fig. $7, \mathrm{n}^{\circ} 2$ et tabl. V). La présence de celle-ci constitue une preuve formelle de la 
Tabl. IV - Saint-Denis, fossé 976. Extrémités de métapodes de bovins,

déchets résultant de la récupération de la matière première (mesures en $\mathrm{mm}$ ) : Bp, largeur proximale;

Dp, épaisseur proximale; Bd, largeur distale; Dd, épaisseur distale; p-a, postéro-antérieur ; a-p, antéro-postérieur (d'après Driesch, 1976).

\begin{tabular}{|c|c|c|c|c|c|c|c|c|c|c|c|}
\hline $\mathrm{N}^{\circ}$ US & Pièce anat. & Côté & Épiphysé & Hauteur conservée & Bp & Dp & Bd & Dd & Surface externe & Sens du sciage & Esquille \\
\hline $709 / 11$ & métacarpe & G & - & 34 & 60 & 34 & & & intacte & en biais p-a & en relief \\
\hline 709/11 & métacarpe & G & - & 32 & 61 & 38 & & & intacte & en biais $p$-a & en relief \\
\hline $709 / 11$ & métacarpe & D & oui & 61 & & & 57 & 31 & intacte & en biais a-p & absente \\
\hline $709 / 11$ & métatarse & _- & - & 44 & & & & & intacte & indéterminé & absente \\
\hline $718 / 18$ & métacarpe & D & - & 36 & 58 & 37 & & & intacte & en biais p-a & en relief \\
\hline $718 / 18$ & métacarpe & D & - & 37 & 64 & 38 & & & intacte & en biais p-a & en négatif \\
\hline $718 / 18$ & métacarpe & D & non & 43 & & & & & intacte & en biais a-p & en relief \\
\hline $718 / 18$ & métacarpe & D & oui & 72 & & & 77 & 40 & intacte & ant.-post. & en négatif \\
\hline $718 / 18$ & métacarpe & $\mathrm{D}$ & oui & 64 & & & 61 & 32 & intacte & absence de trace & absente \\
\hline $718 / 18$ & métatarse & $\mathrm{D}$ & - & 33 & 53 & 49 & & & intacte & post.-ant. & $1 / 2$ relief-1/2négatif \\
\hline $718 / 18$ & métatarse & D & - & 40 & 50 & 47 & & & intacte & post.-ant. & en relief \\
\hline $718 / 18$ & métatarse & G & - & 37 & 56 & 53 & & & intacte & post.-ant. & en relief \\
\hline $719 / 21$ & métacarpe & D & - & 35 & 58 & 35 & & & intacte & post.-ant. & en relief \\
\hline $719 / 21$ & métacarpe & G & oui & 79 & & & 63 & 35 & intacte & en biais a-p & en relief \\
\hline $719 / 21$ & métatarse & D & oui & 83 & & & 57 & 32 & intacte & ant.-post. & en relief \\
\hline $719 / 21$ & métatarse & G & - & & & & & & coups de couperet & en biais p-a & absente \\
\hline $719 / 21$ & métatarse & G & oui & 90 & & & 59 & 35 & intacte & ant.-post. & en négatif \\
\hline $969 / 2$ & métacarpe & G & - & 35 & 64 & 40 & & & intacte & post.-ant. & en relief \\
\hline $978 / 5$ & métacarpe & G & - & 39 & 62 & 40 & & & intacte & absence de trace & absente \\
\hline $1113 / 10$ & métacarpe & $\mathrm{D}$ & oui & 63 & & & 61 & 34 & stries horizontales & ant.-post. & en relief \\
\hline $1113 / 10$ & métacarpe & D & oui & 54 & & & 55 & 29 & intacte & ant.-post. & en relief \\
\hline $1113 / 10$ & métacarpe & _- & oui & 49 & & & 54 & 28 & stries horizontales & absence de trace & absente \\
\hline $1158 / 3$ & métacarpe & D & - & 39 & & & 62 & 40 & intacte & post.-ant. & en relief \\
\hline $1158 / 3$ & métacarpe & D & - & 35 & & & 63 & 37 & coups de couperet & post.-ant. & en relief \\
\hline $1158 / 3$ & métacarpe & G & - & 32 & & & 63 & 39 & intacte & post.-ant. & en relief \\
\hline $1158 / 3$ & métacarpe & $\mathrm{D}$ & oui & 64 & & & 66 & 35 & intacte & ant.-post. & en relief \\
\hline $1158 / 3$ & métacarpe & G & oui & 51 & & & 63 & 37 & intacte & ant.-post. & en relief \\
\hline $1158 / 12$ & métacarpe & G & - & 33 & 58 & 33 & & & intacte & post.-ant. & en relief \\
\hline $1159 / 1 \mathrm{a}$ & métacarpe & $\mathrm{D}$ & - & 46 & 62 & 38 & & & intacte & post.-ant. & en relief \\
\hline 1159/1b & métatarse & $\mathrm{D}$ & - & 43 & 53 & 50 & & & coups de couperet & post.-ant. & en relief \\
\hline $1159 / 1 \mathrm{c}$ & métatarse & $\mathrm{D}$ & oui & 99 & & & 58 & 35 & 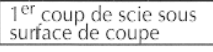 & ant.-post. & absente \\
\hline
\end{tabular}

provenance des déchets d'un atelier de travail de l'os, car la scie n'est jamais utilisée pour la découpe de boucherie aux époques gallo-romaine et médiévale. Le canal médullaire est resté naturel et les côtés correspondant à l'épaisseur de l'os sont cassés suivant le fil de l'os. Les dimensions de ces éclats de débitage sont très variables. Dans certains cas, il est possible de reconnaître l'os d'où ils proviennent. C'est le cas d'une diaphyse de tibia, alors qu'aucune extrémité de cet os n'a été retrouvée.

\section{LES REBUTS DE TAILIE DE BAGUETTES}

Cette catégorie comprend vingt et un rebuts qui présentent soit une extrémité sciée et l'autre cassée, soit deux extrémités sciées (tabl. VI). Les côtés qui correspondent à l'épaisseur de l'os peuvent être sciés ou épannelés. Sur certains exemplaires, il est impossible de reconnaître l'outil utilisé. Parfois, le sciage est incomplet et la présence d'une esquille marque un travail achevé par percussion, d'où une cassure au fil de l'os (710/14 et $718 / 18)$. Il n'est pas toujours possible de déterminer les faces et les épaisseurs, même sur les exemplaires les plus achevés. C'est pourquoi, les quatre pans sont désignés dans le tableau VI par des lettres, sauf lorsqu'ils ont été identifiés, ce qui est alors signifié par un astérisque.

Plusieurs fragments illustrent les différents stades de travail. L'un d'eux (719/21) possède une extrémité sciée et 

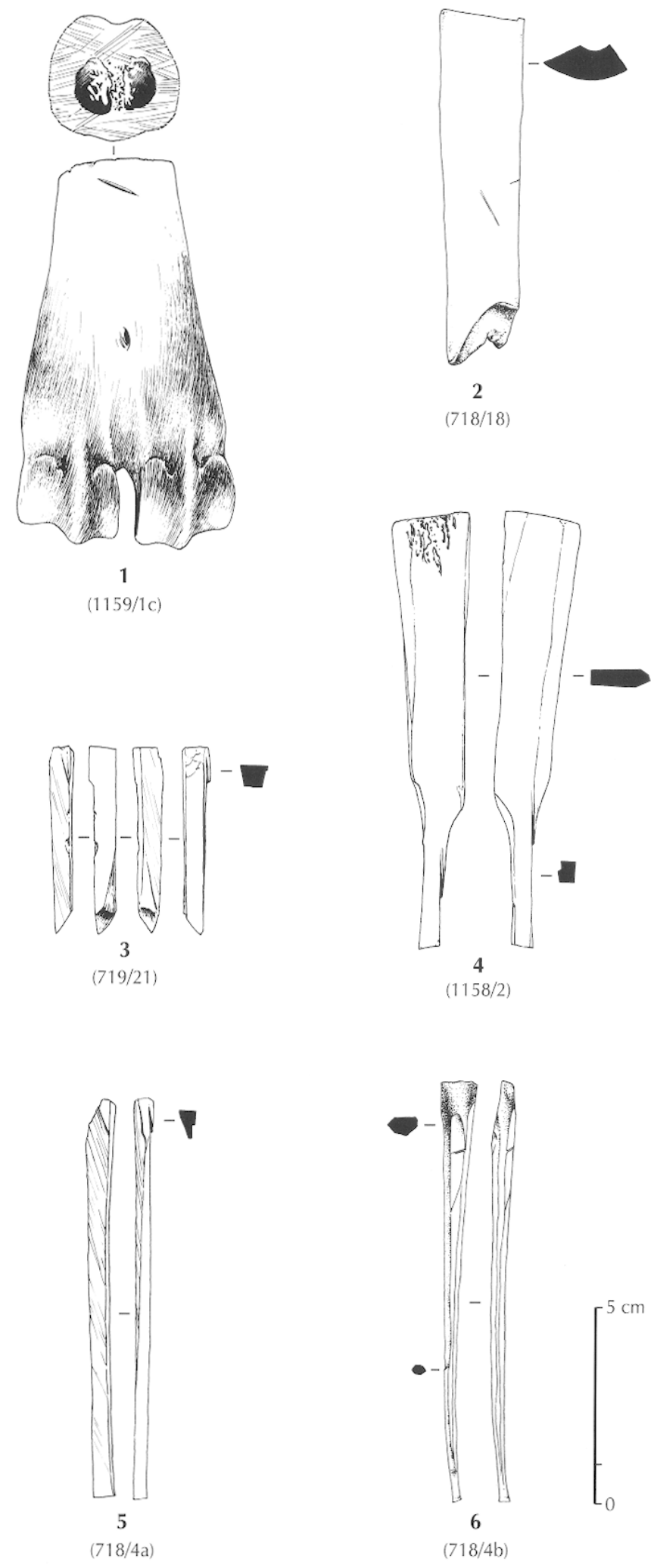

Fig. 7 - Déchets et ébauches d'épingles à tête anthropomorphe stylisée de Saint-Denis : 1-5, déchets ; 6, ébauche (dessins D. Le Bras, unité archéologique de Saint-Denis). l'autre cassée (fig. $7, \mathrm{n}^{\circ} 3$ ). Les côtés sont incomplètement sciés. Des esquilles subsistent à chaque extrémité, indiquant que l'os a été retourné entre les deux sciages. La situation inverse a été observée sur un autre rebut (718/4a) (fig. 7, $\mathrm{n}^{\circ} 5$ ). Les deux côtés correspondant à l'épaisseur de l'os sont sciés dans le même sens ; chacun possède une esquille située à la même extrémité. Un autre fragment de baguette montre que le sciage peut avoir lieu en deux temps (970/2). Les deux pans de coupe sur l'un des bords sont décalés l'un par rapport à l'autre de $1 \mathrm{~mm}$, indiquant une reprise du travail. Un des rebuts provenant d'une plaque de métapode de bovin porte encore l'extrémité d'une baguette équarrie (fig. $7, n^{\circ} 4$ ). Sa face interne a été partiellement nettoyée par épannelage. Cependant, l'os spongieux est encore visible près d'un bord. Les longs côtés de la plaque ont été cassés. Les traces de sciage se trouvent dans l'épaisseur de la baguette. Les côtés présentent une esquille à chaque extrémité indiquant que la plaque a été retournée entre les deux sciages.

\section{LES ÉPINGLES ÉBAUCHÉES}

Une ébauche $(718 / 4 b)$ possède une forme suffisamment élaborée pour assurer le lien entre les déchets et les épingles achevées, confirmant ainsi la fabrication de ce type d'épingles à Saint-Denis (fig. 7, n 6). L'arrière de la tête présente le pan coupé caractéristique et l'emplacement de la face est clairement réservé. Les dimensions sont un peu plus grandes que celles des pièces complètes et laissent donc la possibilité d'achever cette épingle (tabl. VII et VIII). Le corps, grossièrement taillé, est légèrement courbe, ce qui pourrait expliquer l'abandon de la pièce.

\section{LES ÉPINGLES ACHEVÉES}

Quatre des cinq exemplaires achevés sont de même facture et sont de dimensions similaires : 2702/3, 718/2, $718 / 8$ et $719 / 13$ (fig. 8 , n ${ }^{\text {os }} 1-4$ et tabl. VII).

L'épingle complète $718 / 2$ (fig. $8, n^{\circ} 2$ ), la plus fine de toutes dans le rendu des traits, résume parfaitement les différentes caractéristiques : une tête féminine stylisée posée sur deux tores séparés par une gorge. Le pan coupé occupe presque tout l'arrière du crâne et porte deux incisions obliques. Sur la face, la bouche est marquée par une encoche horizontale et le nez. est suggéré par un simple méplat. Les arcades sourcilières sont figurées par un chevron et le décor des tempes montre deux séries de deux incisions en biais et légèrement décalées. Son rejet ne s'explique pas car elle ne présente aucun défaut apparent.

La tête de l'épingle $718 / 8$ (fig. 8, $\mathrm{n}^{\circ}$ 3) porte les traces d'un ratage. Lors du façonnage du tore supérieur, l'outil a ripé et a endommagé sa surface. Cette imperfection n'a été ni rectifiée ni effacée. 
Tabl. V - Saint-Denis, fossé 976.

Éclats provenant du débitage des diaphyses de bovins (mesures en $\mathrm{mm}$ ) : p-a, postéro-antérieur.

\begin{tabular}{|c|c|c|c|c|c|c|c|}
\hline $\mathrm{N}^{\circ}$ US & Provenance & $\begin{array}{c}\text { Longueur } \\
\text { totale }\end{array}$ & $\begin{array}{c}\text { Largeur } \\
\text { au bord scié }\end{array}$ & $\begin{array}{c}\text { Épaisseur maximale } \\
\text { de la corticale }\end{array}$ & Surface externe & Sens du sciage & Esquille \\
\hline $710 / 14$ & métatarse gauche de bovin & 70 & 80 & 10 & intacte & en biais p-a & en négatif \\
\hline $718 / 18$ & face plantaire d'un métatarse de bovin & 83 & 23 & 6 & intacte & absente & absente \\
\hline $718 / 18$ & métacarpe de bovin & 66 & 29 & 10 & intacte & absente & absente \\
\hline $718 / 18$ & métacarpe de bovin & 91 & 24 & 8 & intacte & indéterminé & en relief \\
\hline $718 / 18$ & - & 56 & 10 & 11 & intacte & en biais & absente \\
\hline $719 / 21$ & métacarpe de bovin & 76 & 24 & 7 & intacte & post.-ant. & absente \\
\hline $719 / 21$ & faces caudale et médiale de tibia de bovin & 57 & 48 & 12 & traces de coups de couperet sur la face médiale & indéterminé & absente \\
\hline $719 / 21$ & - & 19 & 17 & 7 & intacte & indéterminé & en relief \\
\hline $978 / 15$ & - & 53 & 5 & 5 & intacte & indéterminé & absente \\
\hline $1113 / 3$ & - & 41 & 11 & - & intacte & visibles & en relief \\
\hline $1113 / 9$ & métapode de bovin & 67 & 22 & 6 & intacte & visibles & absente \\
\hline $1158 / 3$ & - & 53 & 7 & - & traces d'épannelage & visibles & absente \\
\hline $2702 / 11$ & - & 38 & 25 & - & patine & visibles & en relief \\
\hline
\end{tabular}

Tabl. VI - Saint-Denis, fossé 976. Rebuts de taille de baguettes (mesures en mm).

L'astérisque indique que les différentes faces du rebut de taille ont pu être identifiées.

\begin{tabular}{|c|c|c|c|c|c|c|c|c|c|c|c|}
\hline $\mathrm{N}^{\circ}$ US & $\begin{array}{l}\text { Long. } \\
\text { max. }\end{array}$ & $\begin{array}{l}\text { Long. } \\
\text { côté scié }\end{array}$ & $\begin{array}{l}\text { Largeur } \\
\text { côté scié }\end{array}$ & Section & $\begin{array}{l}\text { Extrémité } \\
\text { supérieure }\end{array}$ & $\begin{array}{l}\text { Extrémité } \\
\text { inférieure }\end{array}$ & $\begin{array}{c}\text { A } \\
\text { (ou épaisseur droite *) }\end{array}$ & $\begin{array}{c}\text { B } \\
\text { (ou épaisseur gauche *) }\end{array}$ & $\begin{array}{c}\text { C } \\
\text { (ou surface ext. }{ }^{*} \text { ) }\end{array}$ & $\begin{array}{c}\text { D } \\
\text { (ou canal médullaire *) }\end{array}$ & \\
\hline $710 / 14$ & 34 & 6 & 6 & carrée & sciée & cassée & cassée & $\begin{array}{c}\text { sciée avec esquille et cassée, } \\
\text { se termine en pointe }\end{array}$ & épannelée (2 facettes) & intact & * \\
\hline $718 / 4$ & 32 & 6 & 5 & rectangulaire & cassée & cassée & épannelée & épannelée & sciée & épannelé & \\
\hline $718 / 4$ & 41 & 7 & - & circulaire & sciée & cassée & épannelée & épannelée & épannelée & intact & \\
\hline $718 / 4$ & 99 & 7 & 6 & rectangulaire & sciée & sciée & sciée en biais avec esquille & sciée en biais avec esquille & lisse sans trace & lisse sans trace & \\
\hline $718 / 18$ & 46 & 7 & 6 & rectangulaire & sciée & cassée & sciée & $\begin{array}{l}\text { sciée avec esquille, arrêt } \\
\text { du travail avant la fin }\end{array}$ & $\begin{array}{l}\text { épannelée } \\
\text { avec facettes }\end{array}$ & intact & * \\
\hline $719 / 11$ & 106 & 10 & 9 & rectangulaire & sciée & sciée & sciée en biais avec esquille & cassée & épannelée (facettes) & épannelé & \\
\hline $719 / 21$ & 48 & 7 & 5 & rectangulaire & sciée & cassée & $\begin{array}{l}\text { sciée avec esquille, arrêt } \\
\text { du travail avant la fin }\end{array}$ & $\begin{array}{l}\text { sciée avec esquille, arrêt } \\
\text { du travail avant la fin }\end{array}$ & lisse sans trace & intact & * \\
\hline $719 / 21$ & 77 & 16 & 5 & rectangulaire & sciée en biais & cassée & épannelée & cassée & épannelée & épannelé + intact & * \\
\hline $970 / 2$ & 45 & 5 & - & triangulaire & sciée & cassée & sciée en biais en 2 temps & lisse sans trace & lisse sans trace & - & \\
\hline $970 / 2$ & 79 & 7 & 5 & rectangulaire & sciée & sciée & sciée en biais avec esquille & lisse sans trace & lisse sans trace & lisse sans trace & $*$ \\
\hline $1113 / 3$ & 50 & 7 & 5 & rectangulaire & sciée & cassée & sciée avec esquille & sciée avec esquille & intacte & intact & $*$ \\
\hline $1113 / 5$ & 69 & 10 & 9 & rectangulaire & sciée & cassée & sciée en biais & lisse sans trace & épannelée & intact & * \\
\hline $1113 / 5$ & 74 & 5 & - & triangulaire & sciée & cassée & sciée en biais avec esquille & épannelée & épannelée & - & \\
\hline $1113 / 9$ & 40 & 10 & 4 & rectangulaire & sciée & cassée & lisse sans trace & lisse sans trace & lisse sans trace & lisse sans trace & \\
\hline $1113 / 9$ & 49 & 8 & 5 & rectangulaire & sciée & cassée & sciée & lisse sans trace & lisse sans trace & lisse sans trace & \\
\hline $1158 / 3$ & 40 & 5 & 4 & rectangulaire & cassée & cassée & sciée avec esquille & sciée avec esquille & intacte & intact & * \\
\hline 1158/12 & 30 & 4 & - & triangulaire & sciée & cassée & sciée en biais & lisse sans trace & lisse sans trace & - & \\
\hline $1158 / 12$ & 67 & 9 & 6 & rectangulaire & sciée & cassée & lisse sans trace & lisse sans trace & sciée & lisse sans trace + intact & * \\
\hline $1158 / 12$ & 72 & 5 & 4 & rectangulaire & sciée & cassée & sciée & lisse sans trace & lisse sans trace & lisse sans trace + intact & * \\
\hline $1158 / 2$ & 110 & 21 & 7 & rectangulaire & sciée & sciée & cassée & cassée & $?$ & épannelé + intact & * \\
\hline
\end{tabular}


Tabl. VII - Saint-Denis, fossé 976. Ébauche ( $\left.n^{\circ} 718 / 4 b\right)$ et épingles à têle anthropomorphe stylisée achevées.

Les $n^{o s} 1$ à 9 correspondent aux diverses mesures prises sur les épingles (voir légende fig. 1 ; mesures en mm).

\begin{tabular}{|c|c|c|c|c|c|c|c|c|c|c|c|c|c|c|c|}
\hline No US & État de conservation & Longueur totale & Diamètre & 1 & 2 & 3 & 4 & 5 & 6 & 7 & 8 & 9 & Gorges & Tores & Patine \\
\hline $718 / 4 b$ & complète & 108 & 5 & - & - & - & - & - & 10 & - & 5 & 19 & 0 & 0 & non \\
\hline $718 / 2$ & complète & 112.5 & 3,5 & 17,4 & 1,3 & 1 & 1,4 & 6,1 & 7 & 3,4 & 4,7 & 12,1 & 1 & 2 & non \\
\hline $978 / 3$ & incomplète & 30 & - & 28,6 & 1,8 & 0,6 & 2,3 & 11,7 & 9,5 & 2,4 & 5,9 & 9,8 & 2 & 3 & oui \\
\hline $718 / 8$ & incomplète & 51,3 & 3,4 & 16,9 & 1,7 & 0,5 & 1,7 & 9,2 & 7,5 & 4,6 & 5,4 & 10,3 & 1 & 2 & oui \\
\hline $719 / 13$ & incomplète & 45,8 & 4 & 19 & 1,8 & 0,7 & 1,7 & 5,9 & 8,3 & 3,4 & 5 & 10,6 & 1 & 2 & oui \\
\hline
\end{tabular}

Tabl. VIII - Saint-Denis, fossé 976. Corps d'épingles (mesures en mm).

\begin{tabular}{|l|c|c|c|l|l|l|}
\hline$N^{\circ}$ US & Partie retrouvée & Longueur conservée & Diamètre maximal & Section & Observations \\
\hline $970 / 1$ & corps & 46 & & circulaire & présence de quelques facettes, cassée à chaque extrémité \\
\hline $719 / 12$ & corps & 40 & 4,5 & circulaire & présence de facettes, cassée à chaque extrémité \\
\hline $719 / 12$ & corps & 29 & 4 & circulaire & présence de facettes (la plus large aplatit la section circulaire), cassée à chaque extrémité & peu \\
\hline $1158 / 5$ & corps & 62 & 3,4 & circulaire & cassée à chaque extrémité & non \\
\hline
\end{tabular}

Tabl. IX - Saint-Denis, fossé 976 . Épingles diverses (mesures en $\mathrm{mm}$ ).

\begin{tabular}{|c|c|c|c|c|c|c|c|c|c|}
\hline $\mathrm{N}^{\circ}$ US & $\begin{array}{c}\text { État de } \\
\text { conservation }\end{array}$ & $\begin{array}{l}\text { Longueur } \\
\text { totale }\end{array}$ & $\begin{array}{l}\text { Diamètre } \\
\text { maximal }\end{array}$ & $\begin{array}{l}\text { Hauteur } \\
\text { de la tête }\end{array}$ & $\begin{array}{l}\text { Diamètre } \\
\text { de la tête }\end{array}$ & $\begin{array}{l}\text { Hauteur } \\
\text { du tore }\end{array}$ & $\begin{array}{l}\text { Hauteur } \\
\text { de la gorge }\end{array}$ & Tête & Patine \\
\hline $718 / 1$ & complète & 108,4 & 3,3 & 14,5 & 5,3 & 1,7 & 0,7 & pomme de pin avec double tore & non \\
\hline $718 / 7$ & complète & 107,7 & 3,5 & 15,7 & 5,9 & 2 & 0,6 & pomme de pin avec double tore & peu \\
\hline $718 / 3$ & complète & 107,1 & 3,8 & 9,1 & 8 & $1,4 / 1,7$ & 0,9 & $3 / 4$ de sphère et cône avec double tore & non \\
\hline $620 / 2$ & complète & 80,7 & 5,8 & 3,9 & 7,4 & 3 & 0,6 & tête sphérique avec un tore & - \\
\hline $1158 / 6$ & incomplète & 59 & 6,3 & - & - & - & - & en pointe & peu \\
\hline
\end{tabular}

Tabl. X - Musée Carnavalet, Paris. Ébauches d'épingles à tête anthropomorphe stylisée.

Les $n^{o s} 1$ à 9 correspondent aux diverses mesures prises sur les épingles (voir légende fig. 1 ; mesures en mm).

\begin{tabular}{|c|c|c|c|c|c|c|c|c|c|c|c|c|c|c|c|}
\hline$N^{\circ}$ inv. & État de conservation & Longueur totale & Diamètre & 1 & 2 & 3 & 4 & 5 & 6 & 7 & 8 & 9 & Gorges & Tores & Patine \\
\hline YC $23 / 3$ & incomplète & 52 & 6 & - & - & - & - & - & 9 & 5 & 7 & 11 & 0 & 0 & non \\
\hline YC 23/15 & incomplète & 61 & 4 & - & - & - & - & - & 9 & 3 & 4 & 12 & 0 & 0 & oui \\
\hline YC 23/21 & incomplète & 53 & 5 & - & - & - & - & - & 8 & 5 & 6 & 14 & 0 & 0 & non \\
\hline AY $61 / 7$ & incomplète & 57 & 5 & - & - & - & - & - & 8 & 4 & 5 & 11 & 0 & 0 & oui \\
\hline YC $23 / 33$ & incomplète & 53 & 5 & - & - & - & - & 6 & 8 & 4 & 5 & 14 & 0 & 0 & oui \\
\hline AY $48 / 8$ & incomplète & 48 & 5 & - & - & - & - & 5 & 6 & 3 & 5 & 13 & 0 & 0 & non \\
\hline
\end{tabular}

L'exemplaire 719/13 (fig. 8, $\mathrm{n}^{\circ}$ 4), peut-être également une pièce ratée, présente une cassure au sommet de la tête qui a eu lieu lors de la taille des incisions situées sur le front. L'une d'elles est si proche du bord que ce dernier s'est cassé.

La quatrième épingle (2702/3), façonnée dans le même esprit mais de facture particulièrement grossière, est totale- ment manquée en raison d'un mauvais positionnement du visage sur la matrice (fig. $8, \mathrm{n}^{\circ} 1$ ). En effet, la forme générale de la tête est irrégulière et l'axe du nez est légèrement décalé par rapport à l'ovale du corps. C'est pourquoi tout l'arrière de la tête (pan coupé à deux incisions et partie bombée) est en biais. En revanche, les tores sont dans l'axe, mais sont mal séparés du corps. La bouche, marquée par 


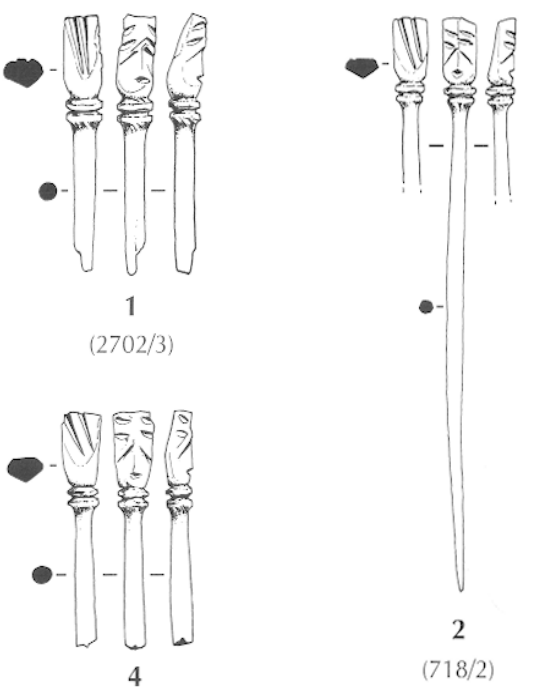

$(719 / 13)$
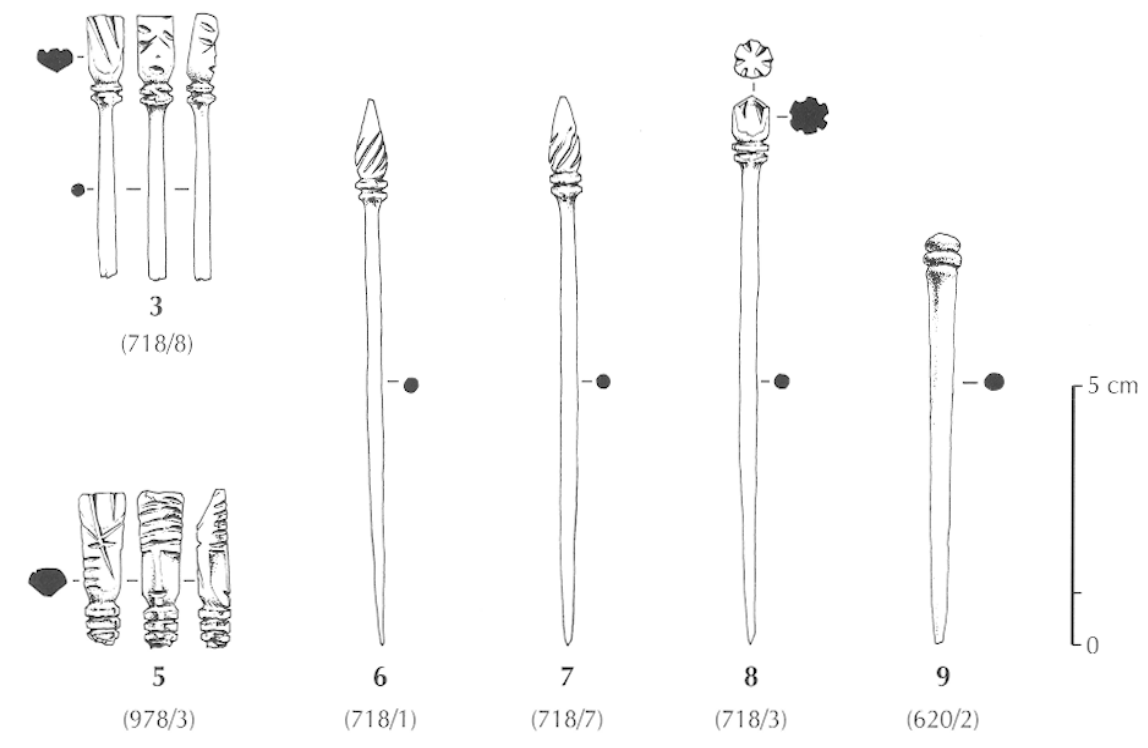

Fig. 8 - Épingles du fossé 976 de Saint-Denis : 1-5, à tête anthropomorphe stylisée; 6-9, autres types (dessins D. Le Bras, unité archéologique de Saint-Denis). une encoche en biais, n'est pas centrée dans le visage. L'arête du nez est plate. Les arcades sourcilières sont marquées par deux incisions non jointives formant un chevron irrégulier.

Le rendu du visage de la cinquième épingle (978/3) est en revanche très différent (fig. $8, n^{\circ} 5$ ). Seuls la tête et les tores sont conservés. Le nez et la bouche sont bien marqués. Sur le front se trouve une série de six incisions continues quasi parallèles et légèrement inclinées, formant une organisation très différente de celle des autres épingles. Le reste de la tête est très anguleux, comme sur les autres exemplaires. Au dos, deux incisions verticales occupent toute la hauteur du pan coupé. Sur la nuque sont taillées deux autres incisions, l'une en biais et l'autre horizontale, ainsi que trois petites marques horizontales qui ne couvrent que la moitié de la largeur.

\section{LES AUTRES ÉPINGLES}

Parmi les cinq épingles à tête géométrique mises au jour (tabl. IX), la plus sobre $(1158 / 6)$ possède une tête en pointe et appartient à un type attesté jusqu'au IV $\mathrm{s}$. (Béal, 1983a, p. 185-186, type A XX, 3). Deux autres (718/1 et $718 / 7)$ possèdent un corps renflé dans son premier tiers, qui s'affine vers la pointe. Leur extrémité supérieure est décorée de deux tores séparés par une gorge, sur lesquels repose une pomme de pin très stylisée, ornée respectivement de six et de sept stries obliques qui s'arrêtent avant le sommet (fig. $8, \mathrm{n}^{\text {os }} 6$ et 7 ). Une quatrième épingle (718/3) possède une tête composée d'une sphère décorée de six encoches sur son pourtour (fig. $8, \mathrm{n}^{\circ} 8$ ). La dernière épingle $(620 / 2)$ dont le corps s'élargit régulièrement de la pointe à son extrémité supérieure porte à son sommet un tore encadré par deux gorges et surmonté d'un cône aplati (fig. $8, \mathrm{n}^{\circ} 9$ ).

\section{LES ÉPINGLES EN OS ET LES REBUTS DE FABRICATION À PARIS (MUSÉE CARNAVALET)}

De nombreux déchets de taille, d'ébauches et d'objets finis en os ont été mis au jour lors de fouilles à Paris ct sont conservés au musée Carnavalet. La localisation et l'origine stratigraphique des pièces provenant du fonds ancien ne sont pas connues avec précision. Tout au plus peut-on supposer qu'elles proviennent des fouilles exécutées au $\mathrm{XIX}^{\mathrm{e}}$ s. et qu'elles forment un ensemble.

L'étude de ce matériel complète les informations apportées par les découvertes de Saint-Denis et permet d'affiner les connaissances sur la technologie mise en œuvre, grâce à certaines ébauches qui illustrent trois stades distincts de l'élaboration du visage.

\section{LES DÉCHETS DE TAILLE}

Le lot est composé de baguettes de section quadrangulaire dont la longueur varie de $45 \mathrm{~mm}$ à $95 \mathrm{~mm}$ environ. Elles portent toutes des traces de sciage et d'épannelage. 
Tabl. XI - Musée Carnavalet, Paris. Épingles à tête anthropomorphe stylisée.

Les $n^{o s} 1$ à 9 correspondent aux diverses mesures prises sur les épingles (voir légende fig. 1 ; mesures en $\mathrm{mm}$ ).

\begin{tabular}{|c|c|c|c|c|c|c|c|c|c|c|c|c|c|c|c|}
\hline$N^{\circ}$ inv. & État de conservation & Longueur totale & Diamètre & $\mathbf{1}$ & $\mathbf{2}$ & $\mathbf{3}$ & $\mathbf{4}$ & $\mathbf{5}$ & $\mathbf{6}$ & $\mathbf{7}$ & $\mathbf{8}$ & $\mathbf{9}$ & Gorges & Tores & Patine \\
\hline YB 27/3 & incomplète & 83 & 5 & 27 & 2 & 1 & 1 & 8 & 12 & 4 & 6 & 14 & 1 & 2 & oui \\
\hline AY 45/13 & incomplète & 33 & 3 & 14 & 1 & - & - & 3 & 6 & 2 & 4 & 9 & - & 1 & oui \\
\hline AY 48/28 & incomplète & 66 & 6 & 14 & 2 & - & - & 4 & 8 & 4 & 5 & 11 & - & 1 & non \\
\hline
\end{tabular}

Deux de ces déchets de taille (AY 50/6 et AY 50/19) portent des esquilles sur chaque extrémité de la baguette, comme sur les exemples dionysiens. Les extrémités d'autres baguettes sont circulaires; elles correspondent certainement à des déchets de matière première abandonnés après sciage de l'épingle finie. Aucun de ces rejets n'indique quel type d'épingles était fabriqué.

\section{LES ÉBAUCHES D'ÉPINGLES À TÊTE ANTHROPOMORPHE}

Six pièces du musée Carnavalet sont décrites comme des ébauches, arrêtées à des stades de travail différents (Dureuil, Béal, 1996, n 2, p. 42). Quatre sont à un stade de façonnage peu avancé, tandis que les deux autres présentent des étapes différentes d'achèvement (tabl. X). Il manque toutefois la toute première opération de fabrication, c'est-à-dire la taille du pan coupé, attestée seulement à Saint-Denis.

\section{Les ébauches arrêtées au façonnage du pan coupé et du corps}

L'ébauche YC 23/15 possède une face postérieure déjà pourvue du pan coupé tandis que le corps est plus ou moins circulaire à la suite d'enlèvements successifs de matière par épannelage. L'os spongieux apparaît à certains endroits. En revanche, la face antérieure est très plate. Sa faible épaisseur ne permet plus de fabriquer ni les tores, ni le visage (tabl. $\mathrm{X}$, mesure 8). Ces défauts sont sans doute la cause de son rejet.

L'ébauche YC $23 / 3$ possède déjà un pan coupé à l'arrière de la tête et un corps arrondi par épannelage. Le sommet de la tête porte des stries laissées par la scie, mais le travail a été achevé sans laisser subsister d'esquille. Le seul défaut apparent est un éclat de matière qui a endommagé le pan coupé au moment de son façonnage, déséquilibrant ainsi l'arrondi de la tête.

L'ébauche YC 23/21 présente une facture similaire. Le sciage au sommet de la tête est inachevé, laissant subsister une esquille sur le bord.

L'ébauche AY 61/7 est la plus achevée de la série. Son corps est circulaire. Le pan coupé et le sommet de la tête sont patinés et ne portent plus aucune trace de sciage. En revanche, le visage n'est pas encore taillé.

\section{L'ébauche arrêtée au marquage des arcades sourcilières et des incisions sur le pan coupé}

Le corps de l'ébauche YC 23/33 est presque circulaire, même si quelques traces d'épannelage sont encore visibles. En revanche, ni tore, ni gorge n'ont été façonnés. Sur la face postérieure, les deux incisions verticales du pan coupé sont mal positionnées et se chevauchent à leur extrémité. Sur la face antérieure, seul le visage est ébauché. Lors de la taille du chevron soulignant les arcades sourcilières un éclat est parti, ce qui a sans doute conduit à l'abandon de cette ébauche.

\section{L'ébauche comprenant le façonnage des arcades sourcilières et des incisions sur le pan coupé, ainsi que la différenciation de la tête et du corps}

La pièce $\mathrm{AY} 48 / 8$ présente les mêmes caractéristiques générales que la précédente. Le tore supérieur n'est pas encore façonné. Seul son emplacement est signalé par une simple incision horizontale. Le pan coupé paraît surdimensionné car il occupe toute la face postérieure, ce qui n'est jamais le cas sur les autres épingles examinées. Un éclat de matière, ôtée sans doute malencontreusement du front, constitue un autre défaut de fabrication.

\section{LES ÉPINGLES}

Trois épingles provenant du fonds ancien du musée (AY 45/13, AY 48/28, YB 27/3, voir Dureuil, Béal, 1996, n 3 , p. 42) ont pu être examinées (tabl. XI et infra catalogue, $\mathrm{n}^{\text {os }} 216-218$, p. 359). Elles diffèrent les unes des autres par leurs dimensions, la forme générale de la tête - plus ou moins aplatie, allongée ou ronde - et par le traitement du visage. Les variantes concernent les incisions situées sur le front. Elles peuvent être disposées en chevrons inversés (AY 45/13), en lignes parallèles horizontales (YB 27/3), ou en biais (AY 48/28). Le nez est concrétisé par une simple arête (YB 27/3) ou par une forme triangulaire donnant un aspect épaté au visage (AY 45/13). En revanche, la bouche a 


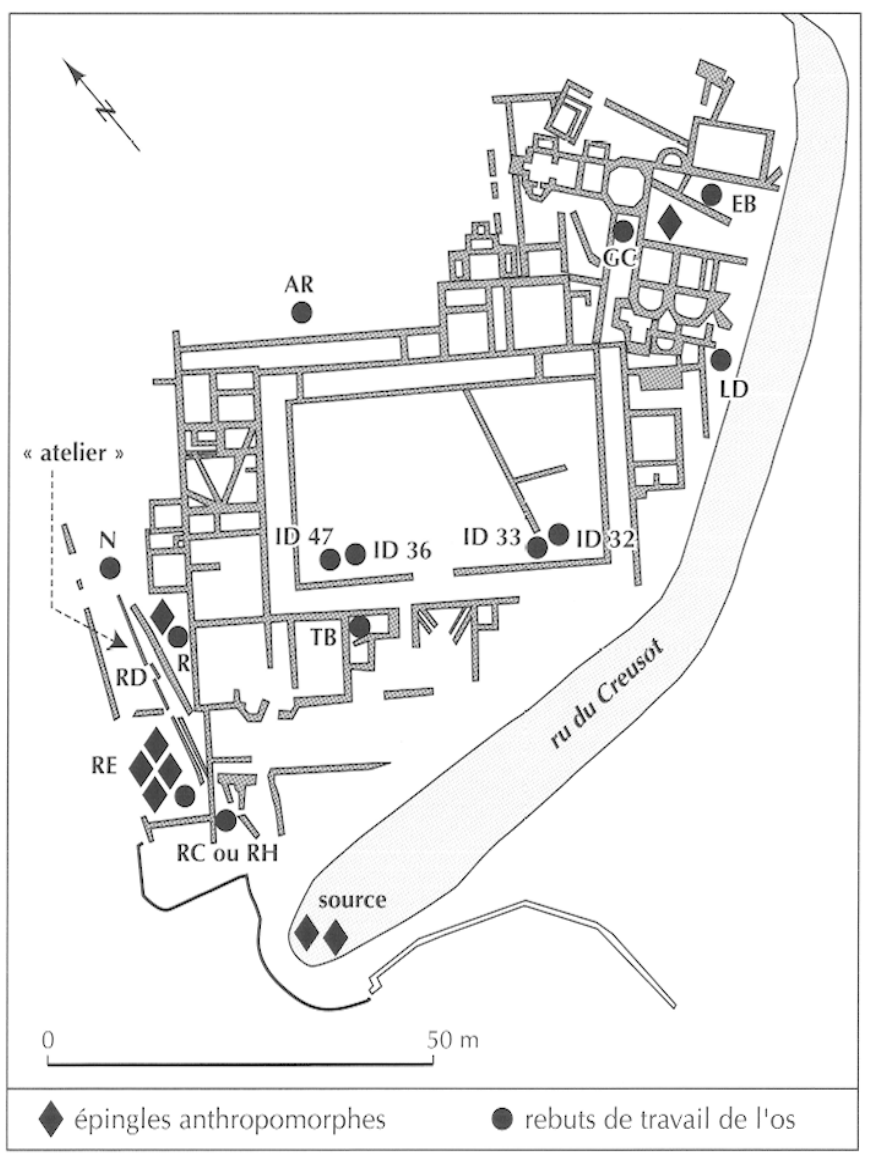

Fig. 9 - Distribution des épingles à tête anthropomorphe stylisée et des rebuts de travail de l'os sur le site d'Escolives-Sainte-Camille (dessin et DAO P. Van Ossel, CNRS).

un rendu toujours identique : une simple incision horizontale légèrement ovale. Sur leur face postérieure, les incisions, généralement au nombre de deux, sont verticales (AY 45/13), légèrement obliques (AY 48/28) ou disposées en chevron, pointe vers le bas (YB 27/3). La tête repose sur un ou deux tores plus ou moins réguliers suite à des enlèvements inégaux de matière (YB 27/3). Ils sont séparés l'un de l'autre par une gorge imparfaitement creusée (AY 48/28 et YB 27/3).

Des traces de sciage sont encore visibles sur un des trois exemplaires (YB 27/3). Seul l'épingle AY 45/13 est patinée et présente un travail soigné.

\section{LES ÉPINGLES D'ESCOLIVES-SAINTE- CAMILLE ET LE PRÉTENDU ATELIER DE TAILLE DE L'OS}

En 1983 est publié un article dans lequel est décrit un atelier de tabletterie découvert à Escolives-Sainte-Camille
Tabl. XII - Provenance des épingles anthropomorphes d'Escolives-Sainte-Camille (les $n^{o s} 202$ à 208

correspondent aux épingles publiées par Prost, 1983).

\begin{tabular}{|l|l|}
\hline$N^{\circ} 202$ & RE, carré 3 (limite RD et R), couche 3, ESC 71 GS 80 (E 88) \\
\hline$N^{\circ} 203$ & limite est de RD et de R, carré E-5, couche 2, ESC 71 GS 157 (E 96) \\
\hline$N^{\circ} 204$ & thermes, ESC 61 PDC 47 (E 77) \\
\hline$N^{\circ} 205$ & provenance inconnue (mais pas « I'atelier »), ESC 68 CDT 8 (E 64) \\
\hline$N^{\circ} 206$ & provenance inconnue, ESC 69 GS 76 (E 73) \\
\hline$N^{\circ} 207$ & provenance espace RE, ESC 71 GS 305 (E 99) \\
\hline$N^{\circ} 208$ & provenance inconnue (sans n inv.) \\
\hline E 402 & inédite, RE, couche 4 (inv. 85 GS 18) \\
\hline E 403 & inédite, RE, couche 4 (inv. 85 CS 19) \\
\hline E 413 & inédite, provenance source, seule la tête est conservée (sans n inv.) \\
\hline E 415 & inédite, provenance source, extrémité de la pointe brisée (sans nº inv.) \\
\hline
\end{tabular}

(Prost, 1983). Ce site, fouillé pendant une trentaine d'années à partir de 1955, est alors connu comme une agglomération gallo-romaine, " $[. .$.$] célèbre pour la$ richesse de ses monuments publics et religieux bâtis aux II $^{e}$, III et IV ${ }^{\mathrm{e}}$ s. après J.-C. " (Prost, 1981, p. 11). Un vaste bâtiment, construit aux abords d'une source, est interprété comme un sanctuaire avec complexe thermal (Petit, Mangin, 1994, p. 77-78). Les travaux archéologiques récents ont montré depuis que les vestiges appartiennent plus vraisemblablement à une vaste villa romaine, occupée jusqu'au V's. au moins (Laurent, 1998, p. 477-479). L'identification d'un atelier de travail de l'os repose sur la présence de rebuts de taille et d'objets partiellement façonnés en os de bœuf, d'équidé ou de cerf trouvés à divers endroits du site. Le lieu de travail est localisé dans une cabane d'artisan construite dans le secteur RD, sorte de cour située à l'arrière de l'aile occidentale de la villa (fig. 9). Les rebuts de fabrication sont peu nombreux, mais paraissaient néanmoins suffisants pour inciter $D$. Prost à faire état d'un atelier (Prost, 1981, p. 29 et 1983, p. 265-266). Si cet auteur n'indique pas expressément que des épingles à tête anthropomorphe stylisée ont été fabriquées sur place, la présence de plusieurs exemplaires de ce type parmi les restes recueillis pouvait le laisser supposer. Depuis sa publication, l'atelier de tabletterie d'Escolives-Sainte-Camille est communément repris dans la bibliographie spécialisée.

Au total, onze épingles à tête anthropomorphe stylisée ont été trouvées dans ce site. La première étape de notre démarche a consisté à déterminer leur provenance exacte (fig. 9 et tabl. XII). Il est rapidement apparu qu'aucune ne provient avec certitude de l'espace $\mathrm{RD}$, c'est-à-dire de l'atelier, mais plutôt de ses abords ( $R E$ et $R$ ) pour cinq d'entre elles. Fn fait, dans les différents rapports et notes de. fouilles, il y a souvent confusion entre les espaces RD, RE et 
Tabl. XIII - Provenance des rebuts de fabrication dans le site d'Escolives-Sainte-Camille.

\begin{tabular}{|c|c|}
\hline 1 extrémité proximale de métatarse de bœuf & ESC 79 GS 195, provenance $R$, couche 4 \\
\hline 1 extrémité proximale de métatarse de bœuf & ESC 79 GS 231, provenance R, couche 7 (pathologie osseusc intéressantc) \\
\hline 1 extrémité proximale de métacarpe de bœuf & ESC 79 GS 266, provenance R, couche 4 (deux encoches de ratage de sciage) \\
\hline 1 extrémité proximale de métacarpe de bœuf & ESC 79 GS 267, provenance R, couche 4 (à moitié fendue) \\
\hline 1 extrémité distale de métapode d'équidé & ESC 79 GS 194, provenance R, couche 4 \\
\hline 1 extrémité distale de métapode d'équidé & ESC 79 GS 264, provenance $R$, couche 4 \\
\hline 1 extrémité distale de métapode d'équidé & ESC 79 GS 290, provenance R, couche 4 \\
\hline 1 extrémité distale de métapode d'équidé & ESC 79 GS 355, provenance R, couche 7 \\
\hline 1 extrémité proximale de métatarse d'équidé & ESC 85 GS 56, provenance RE, couche 3 (sans doute couche d'incendie) \\
\hline 1 extrémité proximale gauche de métatarse de cerf & ESC 85 GS 26, provenance RE, carré 1, couche 4 \\
\hline 1 extrémité distale de tibia d'équidé & ESC 79 GS 300, provenance $N$ \\
\hline 1 diaphyse de métatarse de bøuf scié aux 2 extrémités & sans $n^{\circ} \mathrm{d}^{\prime}$ inventaire, provenance inconnue \\
\hline 1 diaphyse de métatarse de bœuf avec 2 sillons gravés & ESC 84 CDT 46, provenance AR2 \\
\hline 1 face plantaire de métatarse de bœuf & ESC 79 GS 239, provenance R, couche 4 \\
\hline 1 rondelle de rectification (chute de charnière), inédite & ESC 79270 , provenance $R$, couche 4 \\
\hline 1 extrémité d'andouiller de cerf & ESC 69 GS 126, provenance salle RC ou RH, couche 4 \\
\hline
\end{tabular}

$\mathrm{R}$, qui sont des secteurs voisins d'une même partie de l'édifice. Par ailleurs, les numéros de couches des secteurs $\mathrm{RE}$ et $\mathrm{R}$ ne correspondent que très imparfaitement. Une sixième épingle a été découverte dans les thermes. La provenance de trois autres est inconnue. Enfin, les deux derniers exemplaires ont été trouvés ultérieurement dans les fouilles de la source.

La deuxième étape du travail a consisté à déterminer aussi précisément que possible les contextes stratigraphiques des épingles. Là encore, il est rapidement apparu que ces objets proviennent de couches différentes (couches 2, 3 et 4), dont la correspondance chronologique n'est pas assurée. Soupçonnant un ensemble hétérogène, nous avons décidé de reprendre l'inventaire de toutes les pièces osseuses travaillées, à l'exclusion des objets terminés. Outre les rebuts de fabrication signalés dans les publications de D. Prost (1981 et 1983, p. 295), il est possible de mentionner des déchets de matière première non utilisée (tabl. XIII).

Tous ces éléments indiquent effectivement qu'il y a eu fabrication d'objets en os sur place. Toutefois, ils sont peu nombreux (13 pièces repérées) en comparaison à d'autres ensembles comme celui d'Autun (Rodet-Belarbi, 1999, p. 252) ou même celui de Saint-Denis où les chutes et les copeaux sont en plus grande quantité. Ils sont répartis dans des contextes différents, dont l'homogénéité chronologique reste à prouver, et sont dispersés sur l'ensemble du site (fig. 9). Aucun rebut ne provient de l'atelier RD, mais uniquement des espaces voisins $\mathrm{R}$ et RE. Par ailleurs, si certains rebuts (Prost, 1983, p. 295, $\mathrm{n}^{\text {os }} 25,26$ et 27) correspondent effectivement à des ébauches d'épingles aban- données, rien ne permet d'affirmer qu'elles auraient appartenu au type à tête anthropomorphe. Des éléments de charnière et sans doute des plaquettes ou des jetons ont aussi été façonnés. L'observation des épingles à tête anthropomorphe stylisée donne l'impression d'un matériel hétérogène par la taille, les détails des visages et la qualité du rendu.

Très peu d'indices prouvent donc l'existence d'un travail de l'os régulier à Escolives-Sainte-Camille. La fabrication ne semble pas dépasser des besoins limités et occasionnels. Celle-ci a pu avoir lieu à divers endroits du site et à différents moments de son occupation. En l'absence d'arguments pertinents, l'hypothèse d'une fabrication d'épingles à tête anthropomorphe stylisée sur place ne peut être démontrée.

\section{ÉTUDE ET COMPARAISONS}

\section{I.A CHAÎNE DE FABRICATION}

Toutes les épingles étudiées sont en os de bœuf. Le premier travail de l'artisan consiste à récupérer la matière première en dégageant les os longs comme les métapodes et les tibias de leurs extrémités. Puis les matrices sont débitées dans le sens de la longueur afin d'obtenir des baguettes. Celles-ci sont épannelées grossièrement pour leur donner une section de plus en plus circulaire.

Le façonnage de l'extrémité supérieure débute par la taille du pan coupé arrière, si caractéristique de ce type d'épingles. Dans un deuxième temps, le visage est ébauché par un enlèvement de la matière au niveau des joues, ce qui permet de dégager un chevron plus ou moins ouvert 
figurant les arcades sourcilières et une arête centrale ou une surface plane marquant le nez. Sa forme dépend donc de la précision du travail. Les incisions du front sont aussi délicates à effectuer car des éclats peuvent se détacher, comme le montre certaines ébauches endommagées (Paris, musée Carnavalet, YC 23/33 et AY 48/8). Dans un troisième temps, les incisions du pan coupé sont creusées. Ensuite, la tête est séparée du corps de l'épingle par l'exécution d'une gorge délimitant ainsi le tore supérieur. Il ne reste plus alors qu'à tailler l'encoche de la bouche. L'arrondi du menton et le cou sont façonnés par enlèvements successifs de matière. Les traces laissées par l'outil sont très souvent visibles.

\section{TYPOLOGIE}

La schématisation du visage rend au premier abord les têtes fort semblables. En effet, plusieurs caractères sont invariables: le sommet horizontal de la tête, le pan coupé à l'arrière de celle-ci, l'emploi d'incisions et d'encoches pour figurer les divers éléments du visage et une tête séparée de la tige par une bague, lointaine imitation du socle mouluré qui soulignait les épingles à buste féminin du Haut-Empire (voir par ex. Santrot et al., 1975, p. 120-121 ; Béal, 1983a, p. 228 ; Crummy, 1995, p. 25, n 443 ; Mikler, 1997, p. 146). Les têtes diffèrent seulement par le nombre de tores, la taille, les proportions et, surtout, le rendu du visage (fig. 10). La longucur totalc des cxcmplaires entiers oscille entre $57 \mathrm{~mm}$ et $130 \mathrm{~mm}$. La hauteur de la tête (tores inclus) varie de $9 \mathrm{~mm}$ à $29 \mathrm{~mm}$, soit du simple au triple, mais la plus fréquente est comprise entre $12 \mathrm{~mm}$ et $19 \mathrm{~mm}$. Les épingles possédant deux tores ( $56 \%$ des 103 épingles pour lesquelles nous disposons de cette information) sont à peine plus nombreuses que celles n'en présentant qu'un seul (44\%). Un seul exemplaire de tête stylisée repose sur trois tores (SaintDenis) (fig. $8, \mathrm{n}^{\circ} 4$ ) et dcux autres n'en possèdent aucun (Escolives-Sainte-Camille, E 77 et Maastricht).

Les variations observées dans l'agencement des diverses incisions et encoches du visage ne permettent pas de classer ces épingles en sous-types. Tout au plus observe-t-on des façons de faire plus fréquentes (fig. 11). La principale comprend des arcades sourcilières en chevron ( $\mathrm{C}-\mathrm{l}$ ou $\mathrm{C}-2)$, des incisions frontales en chevrons parallèles (A-3 et A-4), en biais (A-5 et A-6) ou inversés (A-7) par rapport aux arcades sourcilières (par exemple Saint-Denis, voir fig. 8, $\mathrm{n}^{\text {os }} 1-4$ et fig. $\left.10, \mathrm{n}^{\text {os }} 1-4\right)$. Une deuxième façon de faire regroupe les épingles dont les arcades sourcilières sont traduites par un trait horizontal (C-3) ou par des incisions frontales le plus souvent horizontales (A-1 et A-2) (fig. 8, $\mathrm{n}^{\circ} 5$ et fig. 10, $\left.\mathrm{n}^{\text {os }} 5-13\right)$. Enfin, la dernière concerne les spécimens possédant une seule incision horizontale couvrant tout le front et les tempes (A-8, par exemple

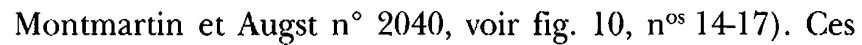
différentes façons sont largement réparties géographiquement. Elles peuvent d'ailleurs coexister dans une même sërie mise au jour sur un même site. C'est le cas pour les exemplaires d'Augst (Deschler-Erb, 1998, p. 139), de Bennecourt (Bourgeois dir., 1999, p. 121), d'EscolivesSainte-Camille (Prost, 1983, p. 263), de Saint-Denis (fig. 8) et de Gautor à Mainz (Mikler, 1997, p. 146, nos 7, 9 et 11). Cette diversité des têtes est le meilleur indice d'une multiplicité des lieux de façonnage, malgré l'unicité du modèle.

Les dissemblances entre les épingles fabriquées en un même lieu, comme à Saint-Denis, sont-elles dues à des " mains " différentes ou à un échelonnement de leur fabrication dans le temps ? La première explication implique un savoir-faire distinctif et suppose plusieurs artisans dans le même atelier. À l'inverse et dans le cas de réalisations échelonnées dans le temps, seule une perte de souvenance des détails du modèle précédent peut expliquer les variations. L'analyse technique apporte quelques éléments de réponse. Les têtes des épingles sont sculptées. L'artisan procède par enlc̀vcments de matière à l'aide d'un instrument à lame tranchante. Avec une telle pratique, la standardisation est évidemment moins facile que pour une épingle tournée. A priori, il est donc difficile de répondre à la question du nombre d'artisans dans un même atelier. Toutefois, la faible quantité de déchets de taille à Saint-Denis suggère une activité peu développée et de courte durée, ne concernant qu'un nombre réduit d'épingles. Cette constatation ne plaide pas en faveur d'une multiplicité d'artisans. En outre, l'idée d'un échelonnement de la fabrication paraît également peu vraisemblable. Un atelier avec une activité permanente aurait dû produire beaucoup plus de rejets. Cette remarque peut s'appliquer à de nombreux sites sur lesquels le travail de l'os et du bois de cerf est mis en évidence : Maastricht (Dijkman, Ervynck, 1998, p. 11-23), Autun (Rodet-Belarbi, 1999, p. 252), Sagalassos (De Cupere, 2001, p. 147-153) et Augst (Deschler-Erb, 1998, p. 69 sqq.). Le nombre relativement restreint d'épingles (300) recensé sur un territoire aussi vaste semble également suggérer une faible production.

\section{VARIÉTÉ DES MATÉRIAUX}

Quasiment toutes les épingles répertoriées sont façonnées en os, matière à la fois bon marché, facile à trouver et à travailler. L'épingle de Léieren à Givenich est l'unique exemplaire en bronze connu à ce jour (voir infra, catalogue, $\left.\mathrm{n}^{\circ} 275, \mathrm{p} .361\right)$. Cette découverte tout à fait exceptionnelle indique que des épingles à tête anthropomorphe stylisée ont été fabriquées dans des matières autres que l'os. On peut penser dès lors que les exemplaires 


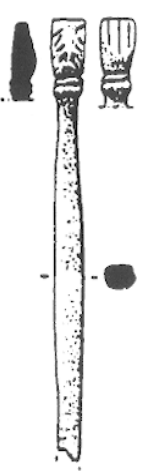

1

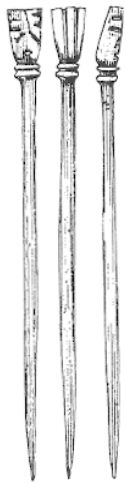

9 10

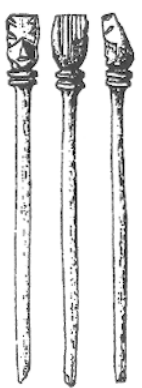

4

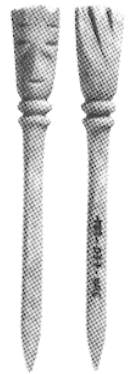

12

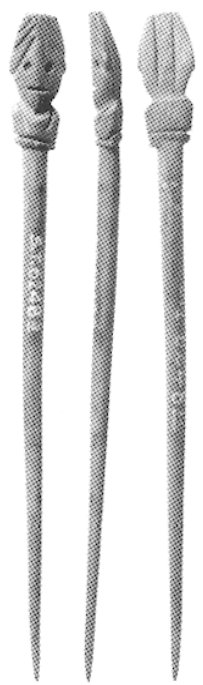

18
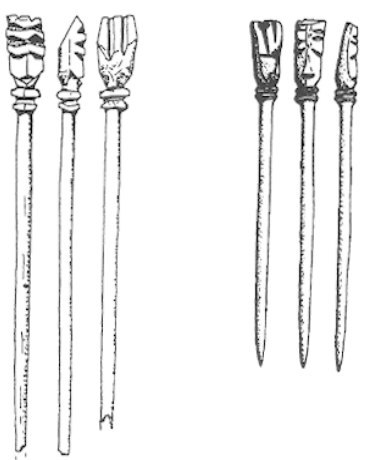

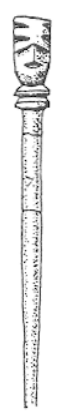

5

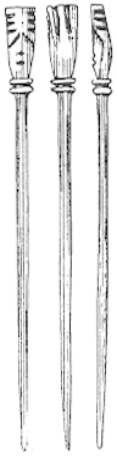

8
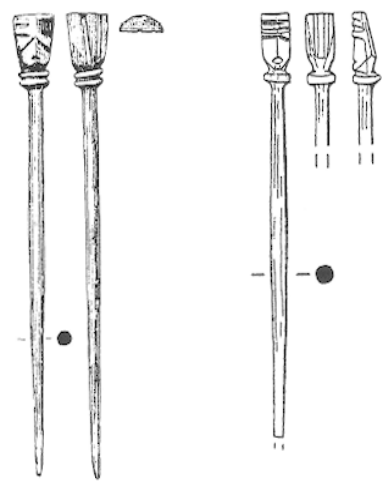

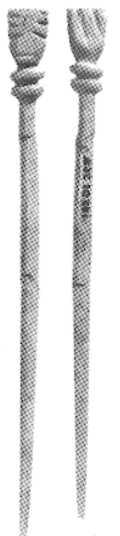

13
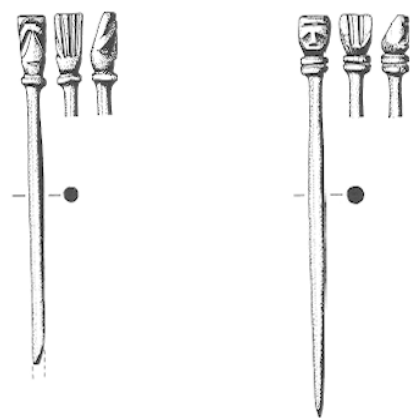

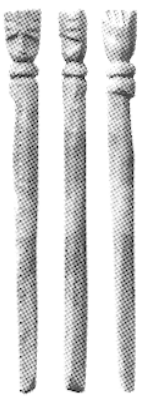

19

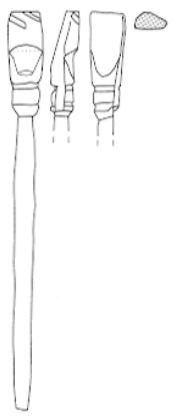

20

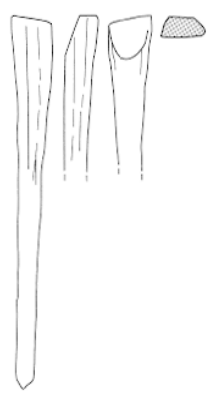

21

Fig. 10 - Épingles à tête anthropomorphe stylisée : 1, Saint-Germain-Laxis (d'après Séguier, 1994); 2, Bennecourt (d'après Bourgeois dir., 1999, $n^{\circ}$ 722) ; 3, Augst, Schmidmatt (d'après Hartmann, 1985); 4, Monheim, Haus Bürgel (d'après Fisscher, 1999); 5 , Bennecourt (d'après Bourgeois dir., 1999, $n^{\circ} 721$ ); 6, Limetz-Villez, cabane Str. 3 (d'après Van Ossel, Ouzoulias, 1987); 7, Traben-Trarbach (d'après Binsfeld, 1973) ; 8-10, Lisieux, tombe 370 (dessins D. Paillard, Conseil général du Calvados); 11, Kaiseraugst, castrum regio XX/X (d'après Riha, 1990); 12, Compans, inv. 182.077 .005 (photo S. Oboukhoff, CNRS); 13, Compans, inv. 182.01.225 (photo S. Oboukhoff, CNRS); 14, 15, Augst, Schmidmatt (d'après Hartmann, 1985); 16, Pithiviers-le-Vieil, Les Oulches (d'après Cribellier, 1993); 17, Worms (@ copyright Stadtarchichiv Worms) ; 18, Trier, Wallstrasse (photo Th. Zühmer, Landesmuseum Trier); 19, Trier, Barbarathermen (photo Th. Zühmer, Landesmuseum Trier) ; 20, 21, Sens, archevêché (dessins P. Van Ossel, CNRS). Échelle: $1 / 2$ excepté $n^{\circ} 16$. 

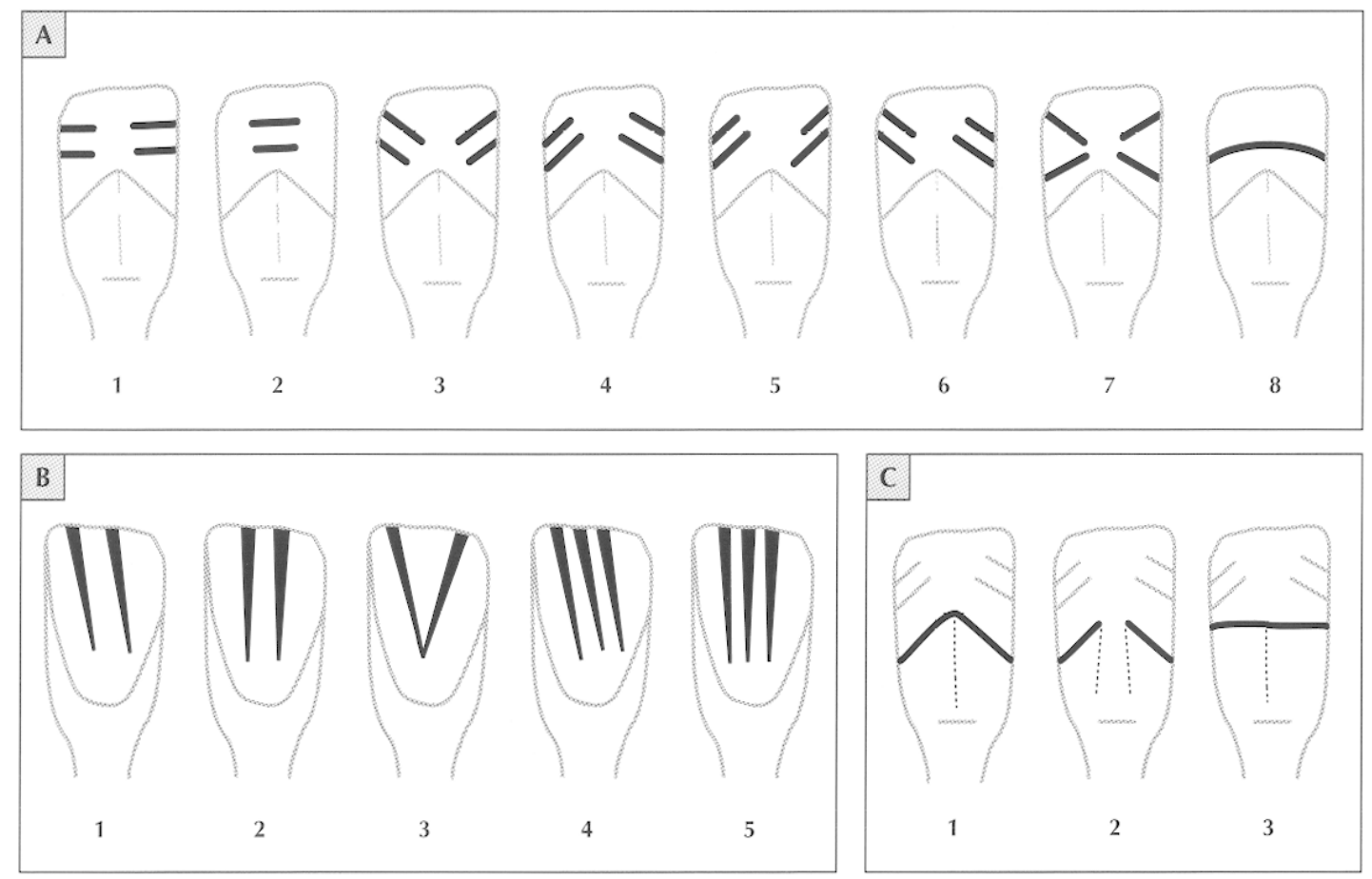

Fig. 11 - Principales dispositions des éléments de décor sur la face (A), sur le pan coupé $(B)$, ainsi que pour les arcades sourcilières $(C)$ : $A-1$, un ou plusieurs traits horizontaux paralleles sur le front et les tempes; A-2, traits horizontaux paralleles sur le front; $A-3$, un ou plusieurs chevrons inversés vers le bas; $A-4$, un ou plusieurs cheorons inversés vers le haut; $A-5$, traits obliques parallèles vers le haut; $A-6$, traits obliques paralleles vers le bas; $A-7$, un ou plusieurs chevrons opposés ; $A-8$, un ou plusieurs traits continus ; $B-1$, double incision oblique; $B-2$, double incision verticale; $B-3$, incision en $V ; B-4$, triple incision oblique; $B-5$, triple incision verticale; $C-1$, arcades sourcilières en cheuron; $C-2$, arcades sourcilières en cheoron interrompu; $C-3$, arcades sourcilieres horizontales (DAO P. Van Ossel, CNRS).

taillés dans cette matière ne représentent qu'une partie de la production de ce type d'épingles.

Quel que soit leur type, les épingles en os sont généralement beaucoup plus nombreuses que les exemplaires en bronze, en argent ou en or, par exemple. Cette fréquence est sans doute liée à leur faible valeur intrinsèque et à leur fragilité. L'existence d'épingles en matériaux plus nobles est pourtant assurée par les sources écrites et par les découvertes archéologiques. Une épingle décorée de perles est ainsi signalée chez Ulpien (Digeste, 34, 2, 25, 10). Plusieurs types d'épingles du IV $\mathbf{s}$. ont été fabriqués dans des matières variées. C'est le cas des épingles à tête polyédrique, nettement plus fréquentes que celles à tête anthropomorphe stylisée. La plupart des exemplaires sont en os ${ }^{9}$, mais de nombreux autres sont en bronze ${ }^{10}$, en argent ${ }^{11}$ et

9. Voir Riha, 1990, p. 109, n² 2475-2477 ; Collectif, 1994, p. 72 ; Bishop, 1996, p. 22 ; Mikler, 1997, p. 141, pl. 29, $\mathrm{n}^{\text {is } 21-24 .}$

10. Voir Collectif, s.d., pl. $16 \mathrm{n}^{\circ} 4$; Collectif, 1984a, p. 163 ; Béal, 1987, p. 201 ; Motteau, 1991, p. 13 ; Collectif, 1994, p. 74 ; Crummy, 1995, p. 29.

11. Voir Goethert-Polaschck, 1977, p. 320 et pl. 24, 256, s-t. même en or ${ }^{12}$, voire en jais ou en lignite ${ }^{13}$. La même variété se retrouve parmi les épingles à tête aviforme dont on connaît des pièces en os ${ }^{14}$, en bronze ${ }^{15}$ et en argent ${ }^{16}$. C'est le cas encore des épingles dont la tête représente une main tenant un fruit, qui peuvent être en os ${ }^{17}$, mais aussi en argent ${ }^{18}$. On peut ajoutcr à cette énumération les épingles à tête ronde ou conique, très simples et très fréquentes en os, mais plus rares en argent (tombes 793 et 1182 à KrefeldGellep ${ }^{19}$ ).

L'existence d'épingles à cheveux en or est aussi attestée, mais les découvertes archéologiques sont très rares. La

12. Voir Planck, 2000, p. 7, fig. 8.

13. Voir Hagen, 1937 ; Crummy, 1995, p. 27.

14. Voir Béal, 1983a, p. 229-230; Mikler, 1997, p. 145, pl. 34, n ${ }^{\mathrm{os}} 6-7$.

15. Voir Pirling, 1974, pl. 31, $\mathrm{n}^{\circ} 5$; Collectif, 1983, p. 279, $\mathrm{n}^{\circ} 241 \mathrm{o}$; Béal, 1987, p. 201 ; Riha, 1990, n 1372 ; Collectif, 1994, p. 72.

16. Voir Roosens, 1962, p. 19, pl. VIII et IX.

17. Voir Béal, 1983a, p. 226, type A XXI, 6 ; Riha, 1990, nº 1367-1371.

18. British Museum, inv. 1934.12-10.21, provenance London. Walbrook. 19. Voir Pirling, 1966, p. 99 et pl. 69 ; p. 137 et pl. 96. 
pratique consistant à dorer à la feuille des têtes d'épingles en os est plus fréquente. Elle est mentionnée dans les sources écrites antiques (Stutzinger, 1995, p. 146, note 58) et connue par des découvertes archéologiques. Une épingle en os du mobilier du sarcophage de l'Aachener Strasse à Trier porte des traces de dorure (Goethert-Polaschek, 1977, p. 320 et pl. 24, 260 k). Sept épingles en os provenant du sarcophage féminin de Dorweiler (Kr. Euskirchen) possèdent une tête dorée à la feuille (Haberey, 1949, p. 88 et fig. 7, $\left.n^{\circ s} 5-6\right)$. On connaît aussi des épingles en bronze doré, comme l'exemplaire à buste figuratif trouvé à Alésia, daté du Haut-Empire (Gaillard de Sémainville, 1983). Cette pratique est motivée par le souci d'imiter les exemplaires plus riches en or et de faire illusion quand l'épingle est fichée dans la chevelure.

La découverte de l'épingle à tête anthropomorphe stylisée en bronze de Givenich suggère une diversité analogue et l'on peut penser que les exemplaires en os répertoriés sont en fait les uniques témoins d'un accessoire de la coiffure féminine beaucoup plus diversifié dans ses manifestations matérielles, dont il ne subsiste que les pièces ayant le moins de valeur.

\section{CHRONOLOGIE DES DÉCOUVERTES}

Le nombre de découvertes datées fournit des éléments de chronologie explicites (tabl. XIV). Toutes appartiennent à l'Antiquité tardive et sont datées entre la seconde moitié $\mathrm{du} \mathrm{III}^{\mathrm{c}} \mathrm{s}$. et la fin du IV ${ }^{\mathrm{e}} \mathrm{s}$. ou le début du Ve $\mathrm{V}$.

L'examen critique des contextes de découverte permet de circonscrire plus exactement l'époque durant laquelle ces épingles furent utilisées. Une fois écartés les ensembles datés uniquement par la durée totale de leur occupation (Dourbes, Furfooz, Saint-Romain) ou les contextes de datation peu précis à l'intérieur du IV es. (Grenoble, Paris, Pfyn, Winchester, Windisch), on note un nombre élevé d'épingles mises au jour dans des niveaux de destruction ou d'abandon du milieu ou du deuxième tiers du IV' $s$. (le puits du sanctuaire de Châteaubleau, Dalheim, Dambron, Mehring, Pithiviers-le-Vieil, Richborough, Traben-Trarbach, Wittlich, Wolkrange-Sesselich, Zouafques). L'association d'épingles à tête anthropomorphe stylisée avec des séries monétaires de la première moitié du IV s. à Augst/ Schmidmatt, à Bennecourt, à Châteaubleau et à Kaiseraugst, ainsi que leur présence dans des sépultures de la première moitié du IV $\mathbf{s}$. à Lisieux et à Reims établissent clairement une correspondance entre ces objets et l'époque constantinienne au sens large.

L'apparition de ces épingles est plus délicate à déterminer. Le contexte le plus ancien est le dépotoir de SaintGermain-Laxis, daté de la fin du III ${ }^{c}$ s. (Séguier, 1994, p. 145). Le caractère homogène du matériel trouvé dans cette structure et l'absence de perturbations stratigraphiques paraissent exclure tout risque de contamination avec la couche de colmatage final de la tin du IV $\mathrm{e}$ s. ou de la première moitié $d u \mathrm{~V}^{\mathrm{e}} \mathrm{s}$. qui scelle le comblement primaire. La présence d'une autre épingle à Augst dans un contexte du castrum-regio XX daté - de façon certes moins précise - de la fin du III ${ }^{\mathrm{c}} \mathrm{s}$. ou du début du IV $\mathrm{s}$. pourrait confirmer une apparition précoce de ce modèle d'épingle.

Sa durée d'utilisation paraît assez longue. Sa présence est assurée dans des contextes de la seconde moitié du IVe $\mathbf{s}$. La chronologie du comblement du fossé 976 de Saint-Denis est assez précisément fixée vers le milieu ou le troisième quart du $I V^{\mathrm{c}}$ s. par le nombre et la qualité du matériel trouvé en association avec les épingles et les rebuts de fabrication. De la même époque peut être daté l'abandon du bâtiment en bois de Limetz-Villez (Van Ossel, Ouzoulias, 1987). Enfin, d'autres épingles ont été trouvées dans des contextes de la fin du IVes. ou de la première moitié $d u V^{e} s$. : dans les casernes théodosiennes d'Arras datées de 390-410, sur le site du parvis de la cathédrale de Chartres en association avec de la céramique de la fin $\mathrm{IV}^{\mathrm{e}} \mathrm{s}$--première moitié $\mathrm{V}^{\mathrm{e}} \mathrm{s}$., au Palais épiscopal de Meaux dans un niveau associé à un peigne Böhme type B (Böhme, 1974, p. 122), ainsi qu'à Canterbury, à Rouen, à Sens et à Vert-Saint-Denis. La chronologie de ces découvertes est sans doute moins sûre en raison des risques de contamination des couches plus ancicnncs ct d'unc rćsidualité toujours possible. Une utilisation prolongée de certaines de ces épingles est toutefois vraisemblable, ne fûtce qu'en raison du nombre de contextes tardifs qui les ont révélées.

\section{RÉPARTITION GÉOGRAPHIQUE}

La répartition géographique des épingles à tête anthropomorphe stylisée est remarquable (fig. 12). La zone de distribution principale correspond aux pays s'étendant entre le Rhin et le Bassin parisien. Des découvertes plus isolées sont connues sur la Loire (Tours, Orléans), sur le Rhône (Vienne) et son affluent l'Isère (Grenoble). L'épingle trouvée dans cette ville constitue aussi l'exemplaire répertorié le plus méridional. À l'est, le Rhin forme une limite très nette. Aucune découverte n'est signalée en Germanie libre ${ }^{20}$. Vers le sud-est, aucune trouvaille ne dépasse le lac de Constance. À l'ouest, les découvertes ne dépassent pas une ligne Lisieux-Tours, excluant la Bretagne de l'aire de répartition. Vers le nord-ouest, la diffusion des épingles a franchi la Manche et atteint l'Angleterre, où sa distribution paraît cependant assez

20. Renseignement M. Erdrich, universitć de Nijmegen (Pays-Bas). 
Tabl. XIV - Datation des contextes ayant liuré des épingles à tête anthropomorphe stylisée.

\begin{tabular}{|c|c|c|c|}
\hline Site & Pays & Type d'occupation & Datation \\
\hline Furfooz/Hauterecenne & B & fortification & occupation fin III $-I \mathrm{~V}^{\mathrm{e}} \mathrm{s}$. \\
\hline Wolkrange-Sesselich/Breden Felder & B & site rural & villa détruite vers 353 \\
\hline Augst/Schmidmatt, Region 17, E, FK B 08451 & $\mathrm{CH}$ & urbain & occupation première moitié IVe s. \\
\hline Kaiseraugst//m Rebgarten & $\mathrm{CH}$ & urbain & couche milieu IVe s. (monnaies et fibule) \\
\hline Pfyn/Städli & $\mathrm{CH}$ & castrum & IVes. \\
\hline Windisch/Vindonissa & $\mathrm{CH}$ & urbain & IVes. \\
\hline Mehring/In der Kirchhecke, Hostertsmorgen & $\mathrm{D}$ & site rural & dépotoir scellé en 353-355 \\
\hline Traben-Trarbach/Gonzlay & $\mathrm{D}$ & site rural & couche de destruction datée de 353 \\
\hline Chartres/Parvis & $\mathrm{F}$ & urbain & occupation fin IVe-première moitié Ve $\mathrm{s}$. \\
\hline Châteaubleau/L'Aumône & $\mathrm{F}$ & sanctuaire & remblai du puits du milieu du IVes. \\
\hline Châteaubleau/La Tannerie & $\mathrm{F}$ & sanctuaire & associée à des monnaies 335-341 \\
\hline Dambron/Fosse Dieppe & $\mathrm{F}$ & site rural & vers $335-340 / 350-355$ \\
\hline Grenoble/Saint-Laurent & $\mathrm{F}$ & urbain & niveau de circulation du IVe $\mathrm{s}$. \\
\hline Limetz-Villez/La Bosse Marnière & $\mathrm{F}$ & site rural & milieu/troisième quart du IVe s. \\
\hline Lisieux/Collège Michelet & $\mathrm{F}$ & tombe 370 & deuxième quart du IVe $\mathrm{s}$. \\
\hline Meaux/Palais épiscopal & $\mathrm{F}$ & urbain & niveau de la seconde moitié du IVe $\mathrm{s}$. \\
\hline Saint-Germain-Laxis/Le Climat des Terres Noires & $\mathrm{F}$ & site rural & fin du III $s$. \\
\hline Saint-Romain/Le Verger & $\mathrm{F}$ & site rural & occupation des III et IVe $s$. \\
\hline Sens/Evêché & $\mathrm{F}$ & urbain & seconde moitié IVe /début Ve $\mathrm{s}$. \\
\hline Vert-Saint-Denis/Les Fourneaux & $\mathrm{F}$ & site rural & fin IVe /début Ves. \\
\hline Zouafques/Wolphus & $\mathrm{F}$ & site rural & comblement de la cave après $346-350$ \\
\hline Canterbury/Marlowe Car Park & GB & urbain & période 4 II (375-450) \\
\hline Richborough/Roman fort & GB & fortification & context $n^{\circ} 1313$, vers 340 et plus tard \\
\hline Winchester & GB & urbain & IV $\mathrm{s}$ \\
\hline Dalheim/vicus & LUX & agglomération & fond de la cave 3 , détruite vers 353 \\
\hline Diekirch/villa & LUX & site rural & occupation surtout IVe $\mathrm{s}$. \\
\hline
\end{tabular}

lâche, couvrant principalement les contrées orientales et méridionales du pays.

À l'intérieur de la zone de répartition principale, un pôle est très nettement marqué par la densité des découvertes sur un espace relativement restreint et par le nombre d'exemplaires signalés: Trier et sa région au sens large, comprenant le sud de la Belgique et le Grand-Duché de Luxembourg. Ce secteur regroupe environ un tiers du total des épingles. Plus à l'ouest, les trouvailles faites le long de la
Seine et de l'Yonne, depuis son cours inférieur (Rouen, Lisieux) jusqu'au Morvan (Autun), forment un axe jalonné par une forte densité de points. Entre ces deux secteurs géographiques, les découvertes sont plus dispersées.

Du point de vue de la géographie historique, cette distribution est centrée sur les provinces du diocèse des Gaules (dicecesis Galliarum) et, dans une moindre mesure, du diocèse des Bretagnes (dicecesis Britanniarum), faisant toutes deux partie de la préfecture des Gaules durant l'Antiquité 


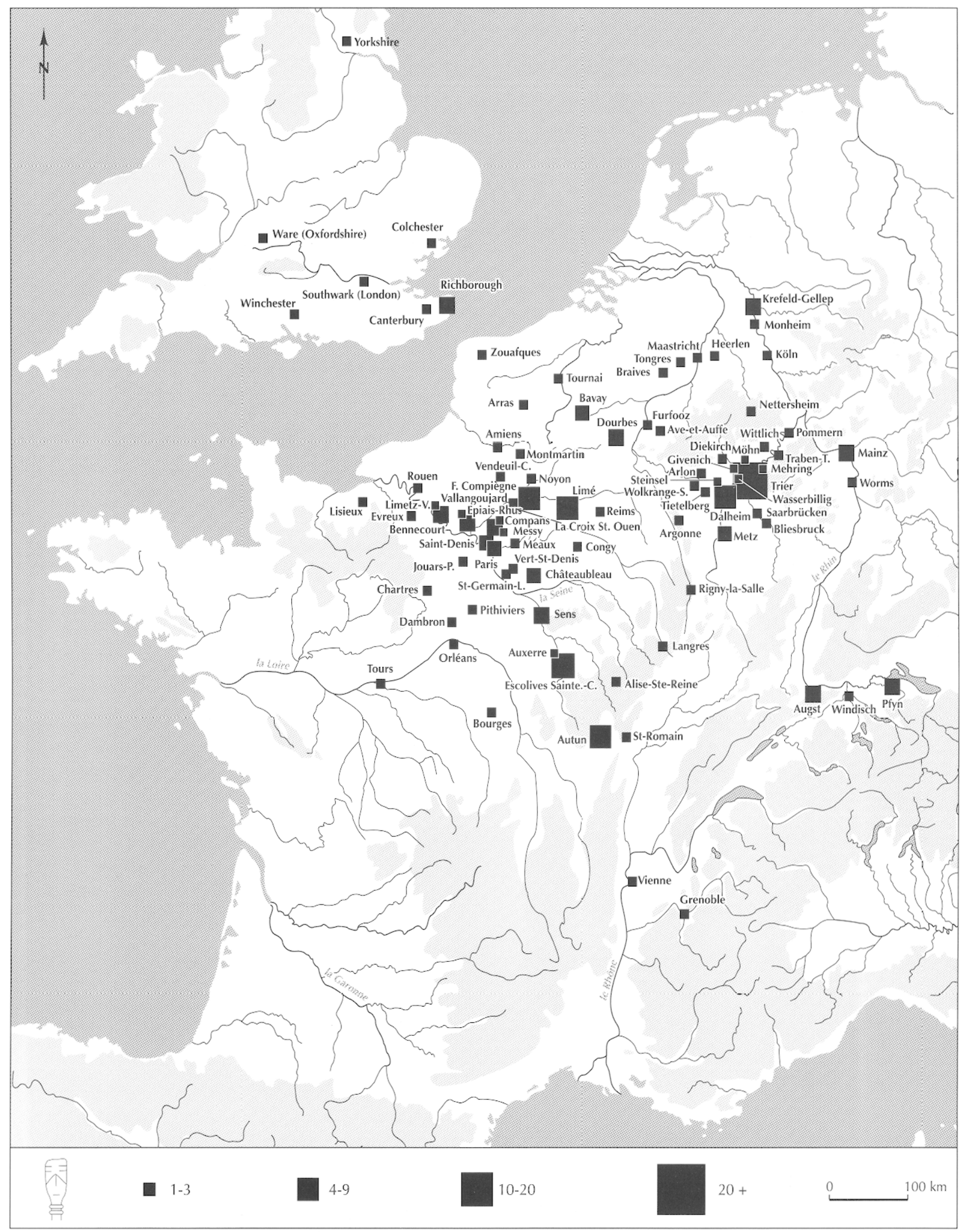

Fig. 12 - Distribution des épingles à lête anthropomorphe stylisée dans les provinces septentrionales et occidentales de l'Empire romain (fond de carte M. Py et M. Feugère, CNRS; DAO P. Van Ossel, CNRS). 


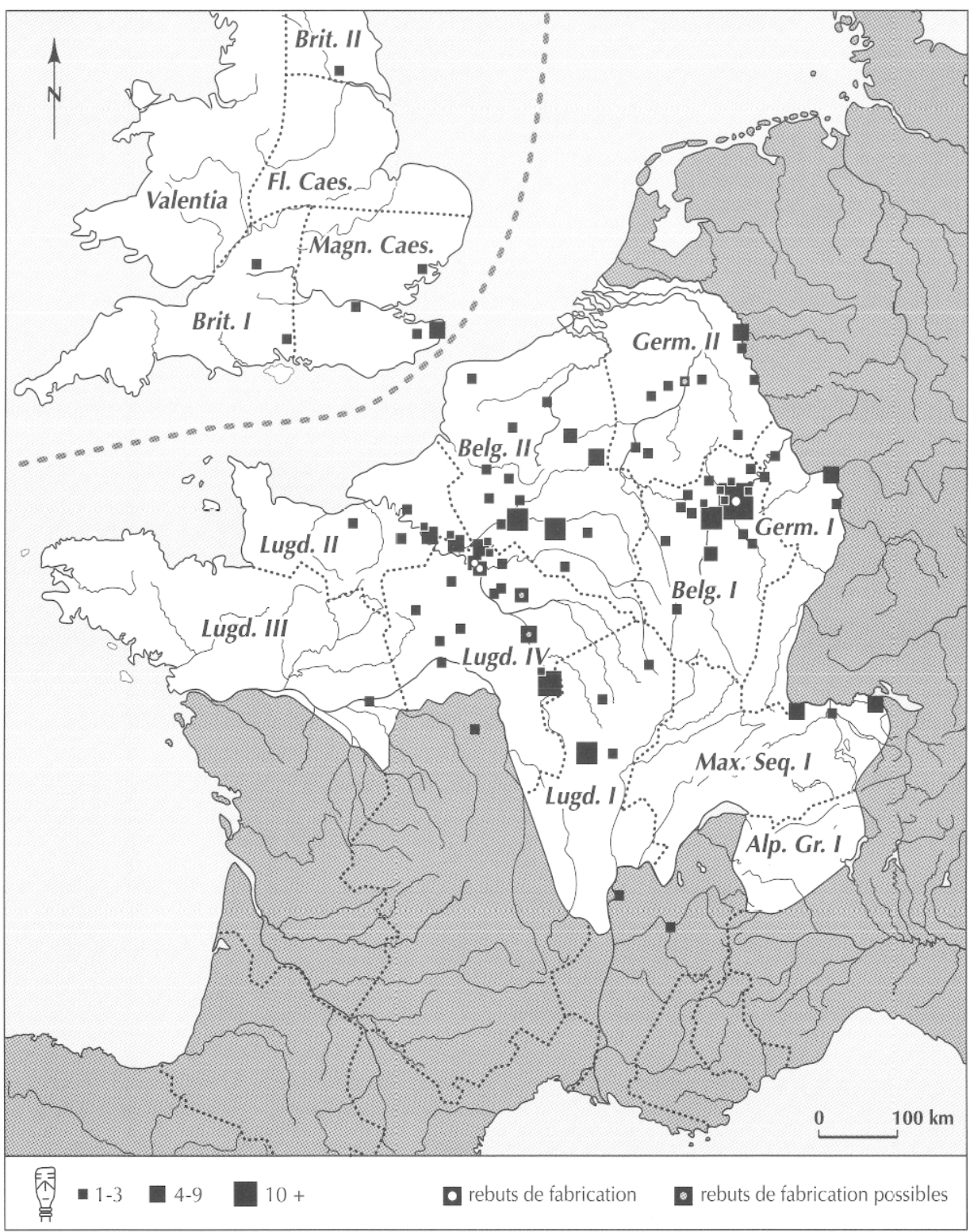

Fig. 13 - Distribution des épingles à tête anthropomorphe stylisée dans le diocèse des Gaules (fond de carte M. Py et M. Feugère, CNRS; DAO P. Van Ossel, CNRS). tardive (fig. 13). Le lien entre la Britannia et les provinces continentales du nord de la Gaule est une réalité bien établie durant l'époque romaine, attestée par la diffusion de nombreux produits manufacturés (voir par exemple: Fulford, 1977 ; Whittaker, 1989, p. 53-77). Presque toutes les provinces de ces entités administratives ont livré des épingles, en nombre variable toutefois. Les rares exemplaires ( 3 sur 300 ) situés en dehors de ce vaste territoire se trouvent sur ses marges (Bourges, Vienne, Grenoble). La raréfaction progressive des occurrences vers les limites occidentales et méridionales du diocèse des Gaules, leur rareté dans les provinces du diocèse de Viennoise et leur absence dans celles de Norique et de Pannonnie, comme en
Germanie libre suggèrent davantage qu'une simple coïncidence entre la distribution constatée et l'aire géographique concernée.

Peut-on pour autant parler d'une aire culturelle homogène ? La question a été posée par Ellen Swift à propos d'autres types d'accessoires vestimentaires de la fin de l'Antiquité comme des bracelets, boucles de ceintures, perles, etc. (Swift, 2000, p. 205-233). Leur distribution géographique met en lumière des manifestations de régionalisme culturel perceptibles surtout dans le vêtement féminin, même dans des régions contiguës. Ce régionalisme, dont la notion doit être préférée à celle d'ethnicité en raison de la difficulté de connaître précisément les senti- 
ments d'appartenance des individus à travers les objets qu'ils portent, peut varier de façon assez sensible selon le type d'affiquet envisagé, soulignant ainsi la diversité et la complexité de telles interprétations. À elles seules, les épingles à tête anthropomorphe stylisée ne peuvent évidemment concourir à définir un espace culturel homogène, d'autant moins que le lien entre une entité administrative aussi élevée qu'un diocèse et un accessoire de la chevelure aussi modeste est, a priori, totalement invraisemblable. Il n'en reste pas moins que de nombreux objets domestiques possèdent une dimension sociale, politique ou régionale et que plusieurs ont des distributions qui couvrent les pays s'étendant entre le Rhin et la Loire, sinon au-delà. Une plus large confrontation des cartes de répartition de différents types de mobiliers déboucherait sans doute sur une meilleure compréhension du phénomène.

\section{VARIÉTÉ DES LIEUX DE TROUVAIILES}

Les épingles à tête anthropomorphe stylisée proviennent de sites de nature variée (tabl. XV), représentatifs des principales formes d'occupation humaine attestées durant la période romaine tardive. Près des deux tiers des épingles ( $60 \%)$ ont été découverts dans des chefs-lieux de cités (Autun, Auxerre, Grenoble, Köln, Lisieux, Mainz, Meaux, Metz, Noyon, Orléans, Paris, Reims, Rouen, Sens, Tournai, Tours, Trier, Southwark, Vienne, Windisch) ou dans des agglomérations secondaires (Alise-Sainte-Reine, Braives, Dalheim, Épiais-Rhus, Forêt de Compiègne, Heerlen, Jouars-Pontchartrain, Pithiviers, Titelberg, Vendeuil-Caply). Le caractère urbain des découvertes d'épingles à tête anthropomorphe stylisée est ainsi clairement mis en évidence. Il n'est donc pas surprenant que celles mises au jour sur les franges de l'aire de distribution principale proviennent presque toutes de villes. Les trouvailles dans les sites ruraux ne sont pourtant pas rares et représentent le quart des lieux répertoriés. Certains établissements sont des villae importantes appartenant manifestement aux élites de la société (Escolives-Sainte-Camille, Limetz-Villez, Wittlich...), mais d'autres sont des habitats d'une très grande modestie, parfois guère plus qu'une simple cabane (Dambron). En fait, dans ce cas encore, toute la gamme des établissements ruraux de l'Antiquité tardive est représentée. Quelques épingles proviennent de fortifications situées sur le limes rhénan (Krefeld-Gellep, Mainz, Monheim), sur le litus Saxonicum (Richborough) ou dans des fortifications de hauteur de la défense intérieure (Dourbes, Furfooz). L'existence de tels accessoires de la coiffure féminine dans des sites militaires est un indice de la présence de familles de soldats dans les lieux de garnison (Bishop, 1991 ; Roxan, 1991 ; Jacques, 1993, p. 197-198).
Tabl. XV - Variété des provenances des épingles à lête anthropomorphe stylisée.

\begin{tabular}{|l|c|c|}
\hline \multicolumn{1}{|c|}{ Nature du site } & Nombre d'épingles & Pourcentage \\
\hline sanctuaire & 14 & $5,5 \%$ \\
\hline fortification & 23 & $9 \%$ \\
\hline établissement rural & 63 & $25 \%$ \\
\hline agglomération secondaire & 36 & $14 \%$ \\
\hline agglomération urbaine & 116 & $46 \%$ \\
\hline Total & $\mathbf{2 5 2}$ & $\mathbf{1 0 0} \%$ \\
\hline
\end{tabular}

Le peu d'épingles trouvé dans des sanctuaires correspond bien avec le caractère domestique de la plupart des contextes de découverte. Cinq exemples seulement sont attestés (Bennecourt, Châteaubleau, Meaux, Möhn, Steinsel) et encore s'agit-il, au moins dans le cas de Bennecourt, d'épingles découvertes parmi les restes d'occupation associés à des foyers domestiques, témoins vraisemblables d'un changement des pratiques cultuelles durant l'Antiquité tardive (Bourgeois dir., 1999, p. 195-198).

En fait, seul le petit nombre de trouvailles dans des installations balnéaires étonne vraiment, car les épingles sont étroitement liées à la toilette et leurs découvertes sont généralement nombreuses dans ces lieux publics (Allason-Jones, 1989, p. 139). Les thermes de Heerlen et les Barbarathermen à Trier sont les seules installations thermales connues à avoir livré quelques épingles à tête anthropomorphe stylisée. C'est là sans doute un autre témoignage de la relative rareté de ce type d'épingles dans l'Antiquité.

\section{INTERPRÉTATION}

Les épingles de l'époque romaine sont étroitement liées à la toilette et à la coiffure féminines. Les sources écrites, les représentations figurées et les découvertes archéologiques fournissent d'abondants témoignages de leur utilisation depuis l'époque républicaine jusqu'à la fin de l'Antiquité (Daremberg, Saglio dir., 1887; Allason-Jones, 1989, p. 137-139 ; Stutzinger, 1995, p. 137-138; Croom, 2000, p. 103).

Dans les textes antiques, le mot latin désignant l'épingle (acus) est souvent accompagné des substantifs qualificatifs comatoria, crinalis, discriminalis, qui désignent à la fois la partie du corps concernée par l'utilisation de l'épingle ainsi que son rôle pour l'arrangement et la fixation de la coiffure chez la femme (acus feminea).

Malgré leurs dimensions réduites, les représentations d'épingles à cheveux sont assez nombreuses dans l'art antique, même s'il n'est pas toujours facile de les distinguer avec certitude d'autres ornements capillaires, comme les 
perles par exemple. Leur identification comme épingle ne fait pourtant aucun doute sur le masque peint de la momie d'Isidora, conservé au musée des Beaux-Arts de Budapest (Geoffroy-Schneiter, 1998), ainsi que sur celui de la momie M 30 du musée de Dresden, ou encore sur le médaillon en verre doré des catacombes de Saint-Pamphile à Rome, par cxemple (Stutzinger, 1995, p. 147-148, fig. 2-3). Les représentations deviennent beaucoup plus fréquentes à partir du milieu du III $s$. pour une raison qui tient moins à une évolution de l'art qu'à celle de la coiffure, dont l'arrangement nécessite davantage d'épingles qu'auparavant (Stutzinger, 1995, p. 143-147). À partir de cette époque, leurs représentations se multiplient dans l'Empire romain, illustrant leur utilisation habituelle comme séparateur des cheveux (acus discriminalis) et comme moyen de fixarion de la coiffure.

Les découvertes archéologiques ont rarement un caractère aussi manifeste. Retrouvés le plus souvent isolés de leur contexte fonctionnel, ces objets ne donnent pas d'informations fiables sur leur utilisation. Seules les découvertes funéraires peuvent apporter des informations sur le port des épingles, mais peu sont suffisamment explicites (Riha, 1990, p. 96 ; Stutzinger, 1995, p. 143, note 47). Dans certaines sépultures à inhumation du $I^{\mathrm{c}} \mathrm{s}$., leur disposition autour du crâne confirme le lien avec la coiffure et permet d'écarter leur utilisation comme fermeture de linceul par exemple, mais elle offre peu de précisions sur leur port et encore moins sur le type de coiffure porté par la défunte. Seul le nombre d'épingles d'un même modèle pourrait être directement en rapport avec l'agencement de la coiffure ou le voile la recouvrant, mais cet aspect de la question n'a guère été approfondi. La sépulture $\mathrm{n}^{\circ} 370$ de Lisieux contenait ainsi trois exemplaires du type anthropomorphe étudié. La riche tombe féminine de Sankt-Matthias à Trier en contenait également trois, dont deux du type étudié, mais leur situation au pied du squelette, parmi les restes de deux coffrets, ne permet pas de les relier directement à la coiffure de la défunte. Les sépultures de Reims et de Maar à Trier ne contenaient sans doute qu'une seule épingle, mais les contextes de découvertes ne sont pas aussi bien connus.

Objet banal de la toilette et de la coiffure féminines, l'épingle s'est progressivement chargée d'une signification dépassant le caractère prosaïque de son usage. Par extension, elle est parfois considérée dans l'Antiquité comme un des symboles de la volupté, par opposition à la virtus masculine représentée par la fibule. Plus généralement, elle est un symbole du statut et du rôle social de la femme et c'est à ce titre qu'elle est souvent représentée sur des scènes sculptées ou figurées. Au IV ${ }^{e}$ s. de notre ère, les scènes de toilette où la femme utilise l'épingle à cheveux, comme sur le coffret de Proiecta (Barbier, 1962), prennent un caractère plus démonstratif. Les classes les plus riches de la société affirment ainsi leur position privilégiée et leur aisance matérielle (Stutzinger, 1995, p. 142). La possession d'épingles en métal précieux participe à ces signes de reconnaissance d'un statut social éminent. Comme tels, ces objets font partie des dons faits à l'occasion des mariages.

Les épingles à tête anthropomorphe stylisée constituent une série bien individualisée au sein d'une famille plus importante, celle des épingles à tête figurative. La particularité de ces dernières est de présenter un buste féminin portant des coiffures qui suivent l'évolution générale de la mode entre la fin de l'époque flavienne et la fin de l'époque théodosienne au moins (Stutzinger, 1995). Les caractères morphologiques de ces épingles à buste féminin et leur diffusion sont étroitement liés à cette évolution. Il y a ainsi des époques où elles sont plus utilisées que d'autres parce que les modèles de coiffures en vogue nécessitent davantage leur utilisation ou parce que l'accumulation d'objets décoratifs dans la chevelure fait partie de la mode. C'est par exemple le cas aux $\mathrm{I}^{\mathrm{er}}$ et $\mathrm{II}^{\mathrm{e}} \mathrm{s}$., époque durant laquelle les épingles à cheveux sont généralement plus longues qu'aux III $^{c}$ et IV IV $^{c}$., parce que les dimensions des coiffures sont beaucoup plus impressionnantes que celles de l'Antiquité tardive (Allason-Jones, 1989, p. 137).

C'est la précision avec laquelle les chevelures sont représentées sur ces épingles qui suggère un lien étroit avec la coiffure figurée, la première étant destinée - au moins théoriquement - à fixer la seconde. Autrement dit, on peut penser qu'il existe un lien préférentiel entre la coiffure représentée sur une épingle à buste et son modèle en vogue à une époque donnée. Comme les épingles à tête anthropomorphe stylisée font partie de la famille des épingles à tête figurative, on peut dès lors penser que leur fabrication et leur diffusion sont également liées à la propagation d'un type de coiffure particulier. Les éléments de chronologie disponibles suggèrent une coiffure répandue à partir de la seconde moitié du III ${ }^{\mathrm{e}} \mathrm{s}$. et plus précisément à partir de l'époque constantinienne jusqu'à la fin $d^{\prime} I^{\mathrm{c}} \mathrm{s}$.

L'évolution générale de la coiffure féminine à partir de l'époque sévérienne est connue par les représcntations monétaires ainsi que par les portraits de la sculpture et de £la glyptique (Evans, 1906; Delbrück, 1933 ; KaschnitzWeinberg, 1936 ; Wessel, 1946 ; Buchholz, 1963; Sydow, 1969 ; Bastien, 1993 ; L'Orange, 1984 ; Wegner, 1984 ; Kersauson, 1996; Croom, 2000). Parmi les nombreuses coiffures en vogue durant l'Antiquité tardive, la Scheitelzopffrisur ${ }^{21}$ ou coiffure " en cimier de casque " était

21. Le mot est difficile à traduire en français. La traduction la plus proche, utilisée dans cet articlc, est coiffure en cimier de casque ou en bonnct phrygien (voir aussi Jedding-Gesterling, 1990 et l'édition française Jedding-Gesterling, Brutscher éds., s.d.). 


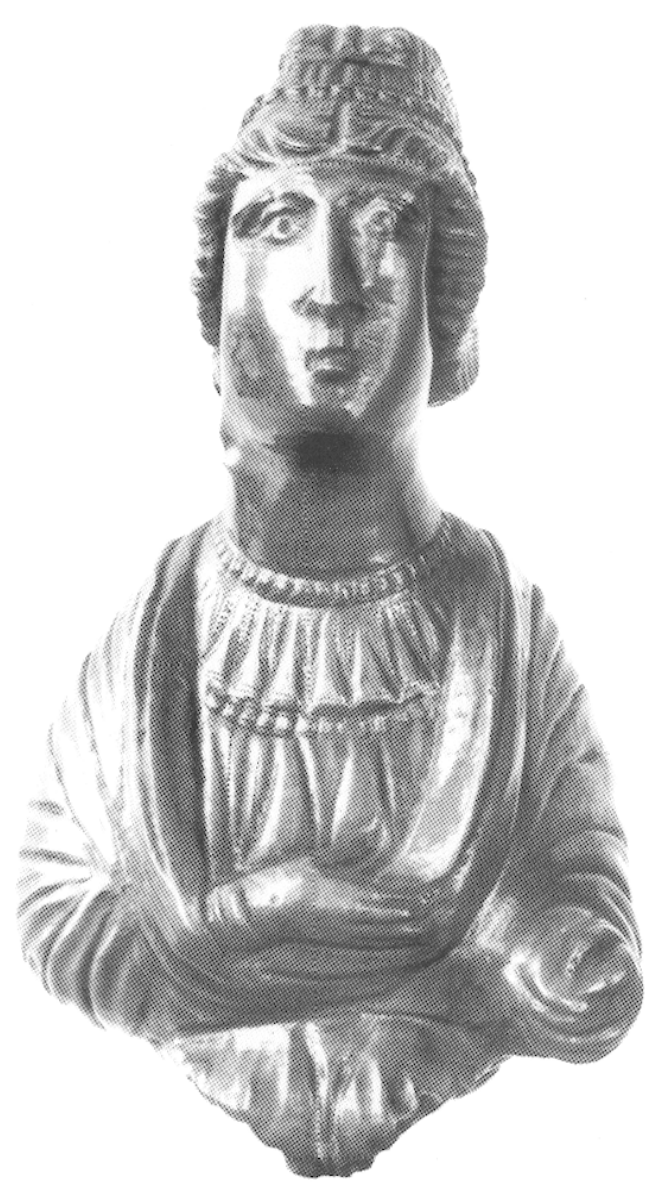

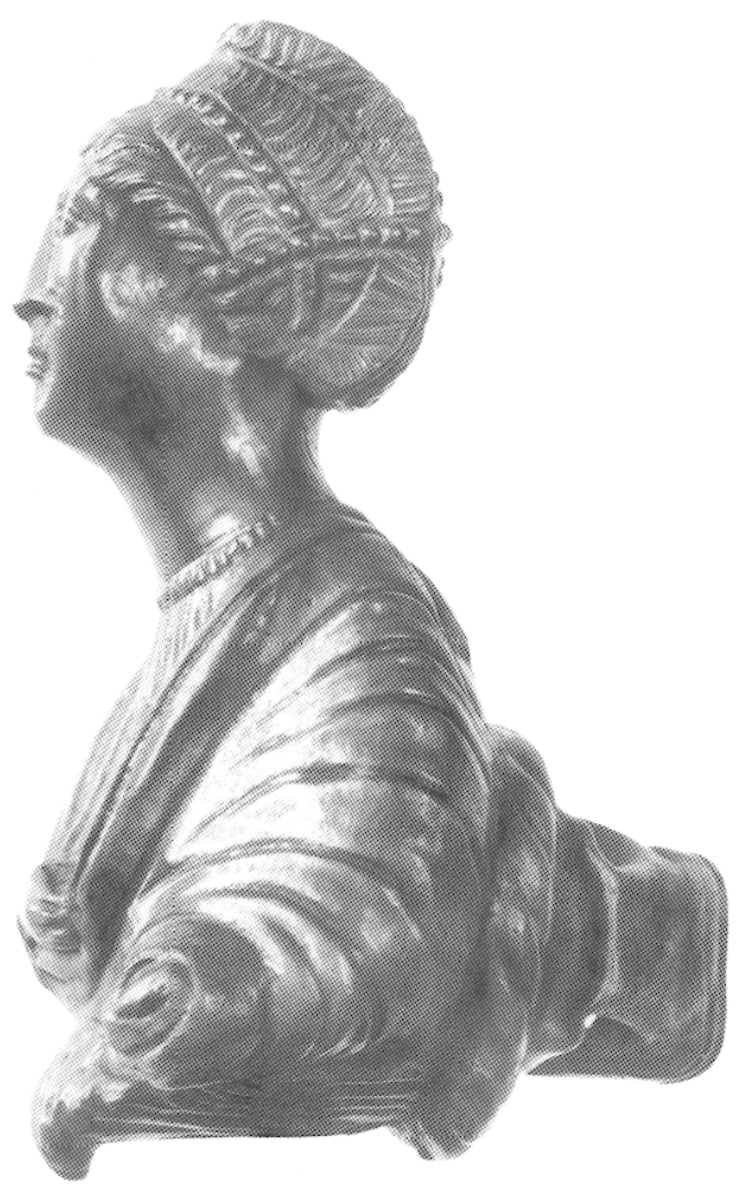

b

Fig. 14 - Statuette en argent de Sirzenich, près de Trier. Hauteur : 13,9 cm (@ copyright RLM, Trier).

particulièrement appréciée, au point de se maintenir durant toute la période malgré des éclipses passagères. La chevelure n'est plus fixée en chignon, mais elle est retournée sur la nuque et remontée en tresses jusque sur le dessus de la tête, où elle se termine en bourrelet (nodus) arrêté par divers procédés de fixation (stéphanè, diadème, bandeau, résille, épinglcs...) qui varient avec le temps. Elle apparaît sur les monnaies de l'impératrice Tranquilina, épouse de Gordien III (241-244) et est utilisée, avec des variations, jusque vers les années 280 . Elle tombe ensuite en désuétude mais ne disparaît sans doute pas complètement. Sa présence sur les monnaies d'Hélène et de Fausta à partir de 324 (Bastien, 1993, p. 618-619) suggère que ces impératrices ont joué un rôle dans son renouveau. Elle reste alors en vogue jusqu'à la fin du $\mathrm{IV}^{\mathrm{c}} \mathrm{s}$. au moins ${ }^{22}$, comme en

22. Il est possible que cette coiffure ait encore été représentée au Ve $s$. sur la procession des Vierges Saintes à Saint-Apollinaire le Neuf, à Ravenne (Heurgon, 1958, p. 65).

\section{témoignent les monnaies d'Aelia Flacilla et de Galla Placidia.}

Plusieurs statuettes et portraits sculptés ou peints portent ce type de coiffure et montrent que le mode de fixation de la chevelure sur le sommet de la tête est fréquemment constitué par des épingles. Celles-ci sont perceptibles sur la très belle statuette d'Aelia Flacilla, conservée au Cabinet des Médailles, où elles sont représentées par quatre petites têtes rondes fixant un voile sur le sommet du bourrelet capillaire (Delbrück, 1933, p. 163-165 et pl. 62). Des épingles sont visibles aussi sur les statuettes en argent de Sirzenich, près de Trier (fig. 14), qui montrent la complexité de ces coiffures élaborées du IV s. (Binsfeld, 1984). Sur le médaillon central de la broche en or du trésor de Ténès (Tunisie), l'impératrice porte une coiffure analogue, dont chaque natte est fixée par une perle ou une épingle (Heurgon, 1958, p. 65). La même coiffure et le même mode de fixation sont clairement idcntifiables sur un portrait d'Égypte peint sur bois, conservé au musée royal de 


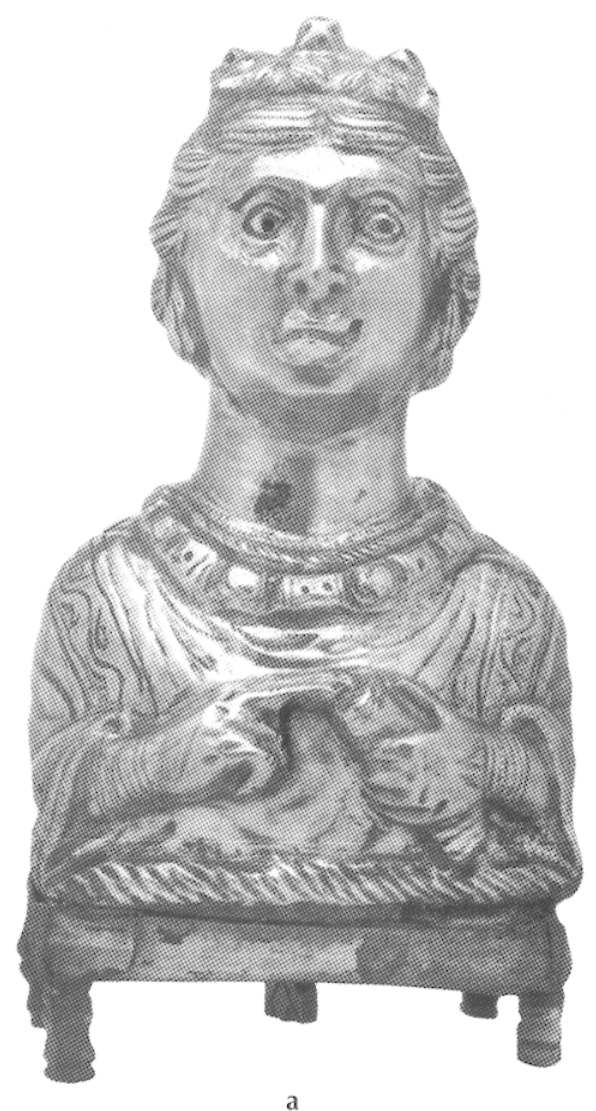

Fig. 15 - «Poìrier» du trésor de Hoxne (@ copyright The British Museum, London).

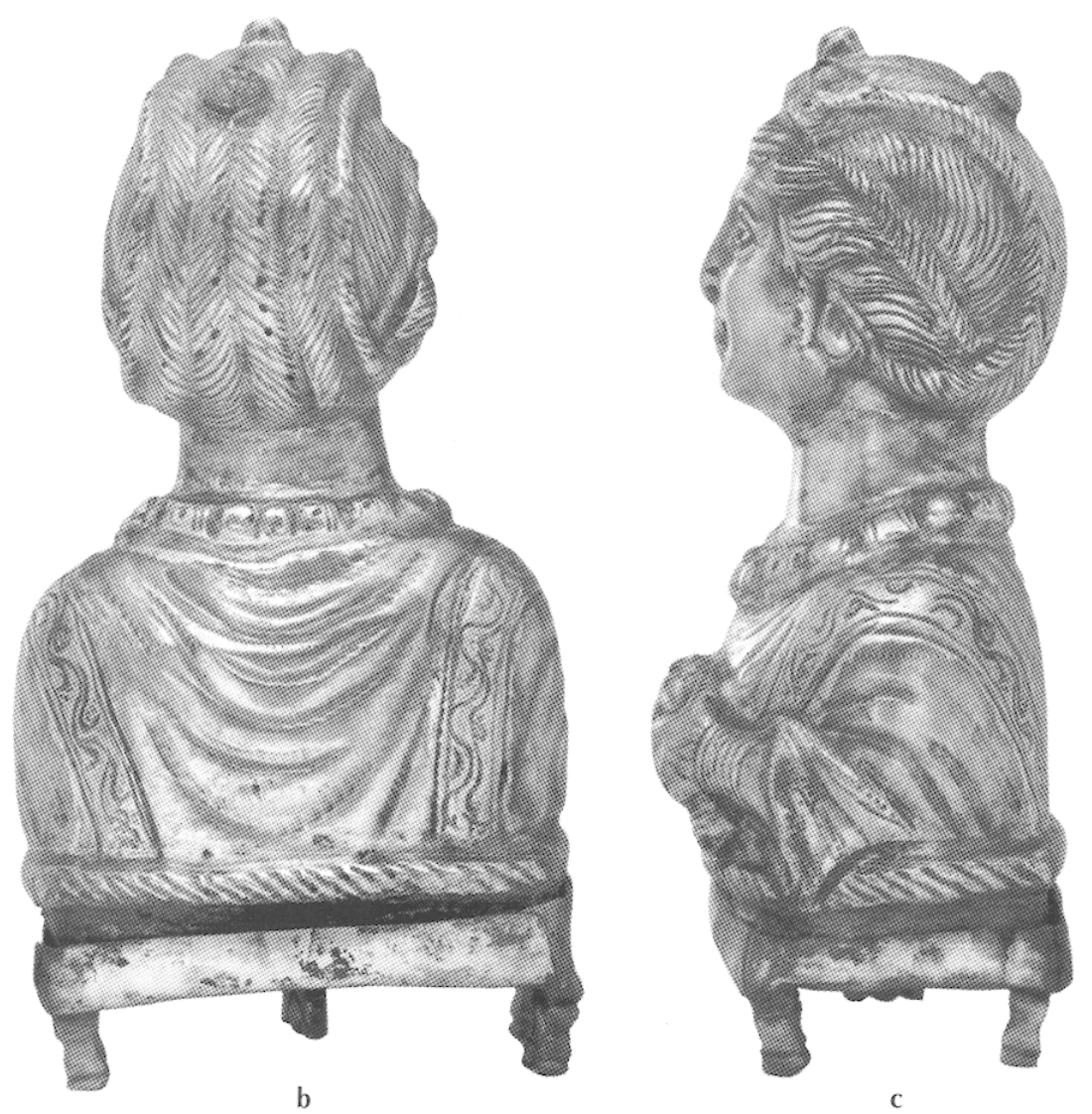




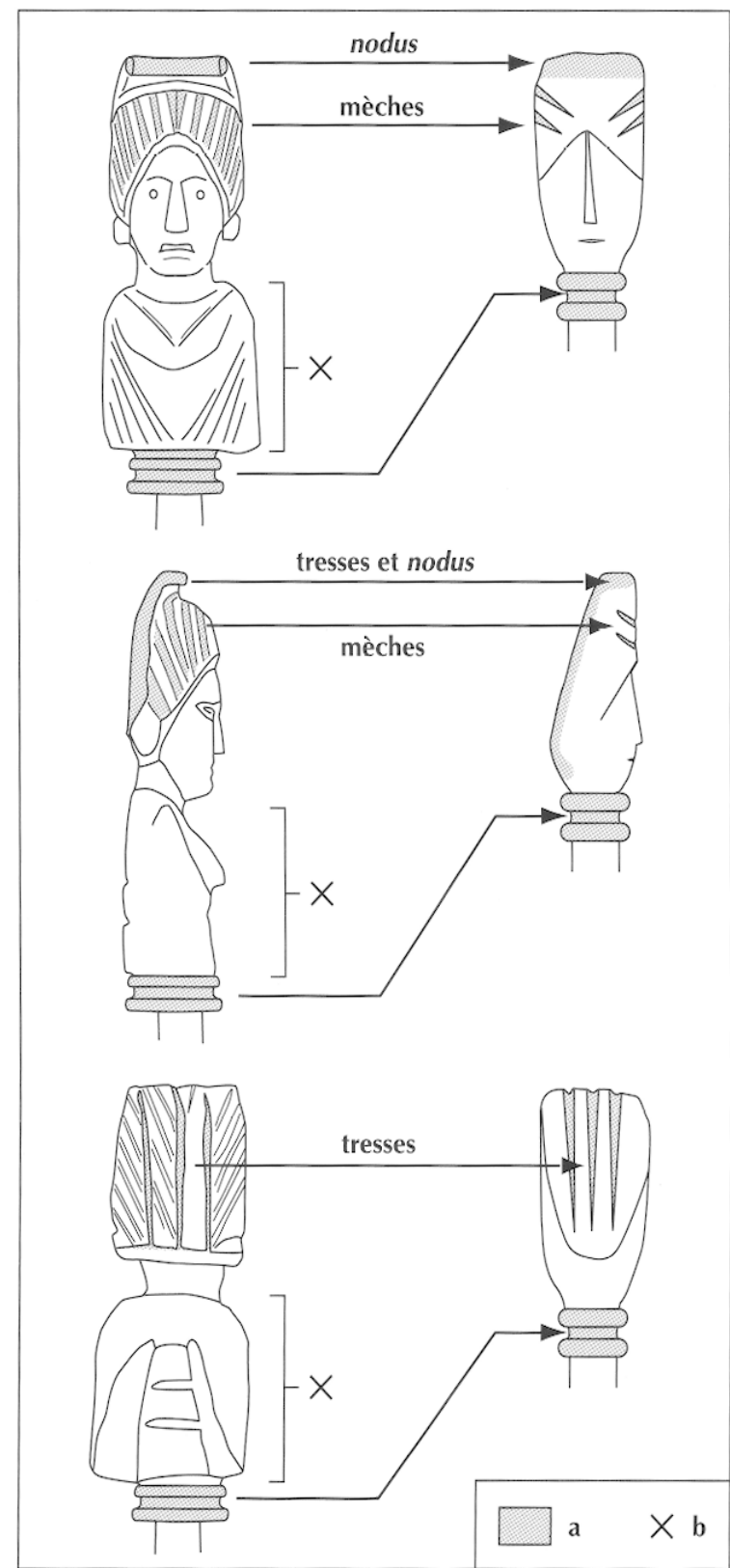

Fig. 16 - Schématisation de l'épingle à buste féminin figuratif vers l'épingle à tête anthropomorphe stylisée : $a$, éléments repris ; b, éléments supprimés (modèle d'après l'exemplaire de Augst, Römermuseum, inv. 24.641 ; DAO P. Van Ossel, CNRS).

relance de la mode à partir du deuxième quart du $\mathrm{IV}^{\mathrm{e}} \mathrm{s}$. et son maintien jusqu'à la fin du $\mathrm{IV}^{\mathrm{e}} \mathrm{s}$. ou le début du $\mathrm{V}^{\mathrm{e}} \mathrm{s}$.

Il reste à expliquer l'extrême schématisation de la représentation de la coiffure sur les épingles. La stylisation poussée n'est pas un phénomène propre à l'Antiquité tardive. Certaines épingles du Haut-Empire le sont également. C'est le cas d'une épingle d'Autun (Béal, 1987,

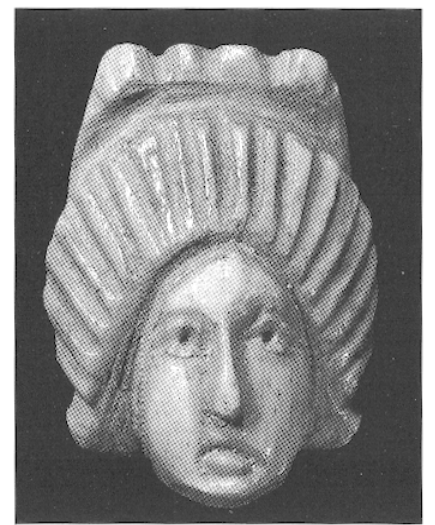

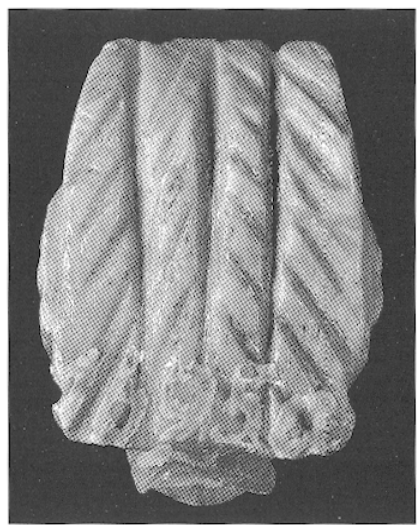

b
Fig. 17 - Épingle à buste féminin provenant d'Italie, Hannover, Kestner-Museum, collection Kestner, inv. 1695a, sans échelle (d'après Stutzinger, 1995, p. 193, fig. 52 ; @ copyright KestnerMuseum, Hannover).

$\mathrm{n}^{\circ} 378 \mathrm{c}$ ) dont le front en demi-cercle est surmonté d'un large espace lisse se terminant par un chignon. C'cst le cas aussi des épingles de Valkenburg (Verhagen, 1993, p. 386, $\mathrm{n}^{\circ} 107$ ) et de Braives (Bulletin de la Société historique et archéologique de Waremme, VI, 1972, p. 20 et pl. II), où les nombreuses incisions sur le front et à l'arrière de la tête permettent de reconnaître sans trop de difficultés des types de coiffure des $\mathrm{I}^{\mathrm{cr}}$ et $\mathrm{II}^{\mathrm{c}} \mathbf{s}$.

La compréhension de la chaîne de fabrication permet d'écarter avec certitude l'hypothèse de maladresses. La schématisation n'est pas davantage liée à la petite taille de la tête car les exemplaires 718/2 de Saint-Denis (fig. 8, $\mathrm{n}^{\circ}$ 2) et AY 45/13 de Paris, par exemple, possèdent un rendu de visage soigné malgré leur très petit format. De la même façon, le modèle paraît trop homogène, trop construit et trop largement diffusé pour accepter l'idée d'une version bâclée des épingles à buste. Tout indique donc qu'il s'agit d'une abstraction délibérée.

Une première explication proposait d'établir un lien avec l'art celte et en particulier avec les représentations de la tête humaine chez les Celtes (Robert, 1976, p. 125-127). De manière générale, cette hypothèse était fondée sur une similitude de facture avec certaines têtes trouvées dans plusieurs sanctuaires de sources, notamment à AliseSainte-Reine, à Chamalières, aux sources de la Seine, à Essarois, à Montbouy, dans la forêt de Halatte, etc. Plus précisément, l'identification des incisions sur le pan coupé avec les doigts d'une main incitait $\mathrm{C}$. Robert à voir une similitude avec les têtes coupées d'Entremont et de Nages ou avec d'autres représentations de " têtes coupées avec imposition de la main dans les cheveux » (id., ibid., p. 127). Cette explication fut souvent reprise par la suite, faute 
de mieux et avec prudence pour ce qui est du symbolisme de la tête dans l'art celte (Dureuil, Béal, 1996). L'hypothèse de C. Robert (1976) et le lien avec l'exaltation de la tête chez les Gaulois ne sont plus recevables, depuis que les travaux de D. Stutzinger (1995) ont montré le lien entre les épingles à tête stylisée et celles à tête non stylisée permettant ainsi de les rattacher à la famille plus large des bustes féminins.

La réfutation de cette explication ne dément pas pour autant les rapports étroits entre la facture des épingles et les traditions artistiques autochtones de la Gaule. Les similitudes avec de nombreuses statues et têtes sculptées sur pierre et sur bois, ou encore avec les têtes de pieu figuré découvertes en Gaule (voir par exemple Deyts, 1983, p. 168-177; Deyts, 1994, p. $34-36$ et 55 ; Collectif, 2000 , p. 9-92; Romeuf, 2000, p. 64) comme en Germanie à l'époque préromaine (Müller-Wille, 1999, p. 26-28), sont à cet égard suffisamment explicites. Les analogies sont frappantes tant dans l'expression générale que dans la technologie employée (taille en méplat à grands coups de ciseau, recours aux incisions pour marquer le regard et la bouche, arête rectiligne du nez...). Le caractère délibérément schématisé de la figuration humaine sur les épingles permet de penser que ce rapprochement n'est pas fortuit et que le rattachement à des expressions culturelles propres à la Gaule a été sciemment recherché. La dimension symbolique, religieuse ou même politique sous-jacente à cette manifestation demeure toutefois obscure. Faut-il la rapprocher d'un rejet de l'image, de toute représentation figurée, selon une tendance qui se manifeste fortement aux $\mathrm{III}^{\mathrm{e}}$ et IV $\mathrm{s}$. dans certains milieux philosophiques ou religieux? (Carrié, Rousselle, 1999, p. 492-496). Cela paraît à vrai dire peu probable, car la schématisation ne supprime pas l'image. Faut-il y voir, plus vraisemblablement, une résurgence culturelle, l'expression d'une fidélité retrouvée à des traditions provinciales ou locales, comme cela a été souvent observé dans diverses régions de l'Empire romain à partir du III s. ? (voir Goodman, in Garnsey, Saller, 2001, p. 85-86). Une réponse demande de préciser le contexte dans lequel l'épingle à tête anthropomorphe stylisée, en tant qu'objet si particulier d'une mode, est apparu.

Un début d'explication peut être fourni par la façon dont les styles de coiffure sont propagés dans le monde antique. À cet égard, le rôle déterminant des princesses impériales a déjà été souligné à plusieurs reprises (Evans, 1906 ; Wessel, 1946 ; Bastien, 1993). Les coiffures figurées sur leurs monnaies ou leurs portraits sculptés sont l'expression d'une mode, suivie par ricochet dans les différentes couches de la société (Bastien, 1993, p. 573). C'est là une manière d'affirmer sa fidélité à la famille impériale et, andelà, à un système politique et social auquel les individus s'apparentent. L'apparition des épingles à tête anthropomorphe stylisée peut être comprise dans une telle perspective. En raison de sa diffusion ne dépassant guère les limites du diocèse des Gaules, son origine doit sans doute être cherchée dans cette région, dans quelque ville jouant un rôle de "phare de la mode ". La ville de Trier, à la fois chef-lieu de la cité des Trévires, métropole de la province de Belgique première, métropole du diocèse des Gaules, siège d'une préfecture du prétoire à partir de la fin du IV s., et sans doute aussi garnison d'un commandement militaire (Heinen, 1984, p. 18-21), vient directement à l'esprit. Cette ville est en outre résidence impériale entre 286-293 (Maximien), puis par intermittence durant le $\mathrm{IV}^{\mathrm{c}} \mathrm{s}$. (Heinen, 1984, p. 21-27). Constance Chlore y séjourne entre 293 et 305, puis Constantin (quand il n'est pas en campagne) et sa famille jusqu'à la bataille du pont Milvius en 316 . Entre 328 et 340 , son fils Constantin II y réside en permanence, d'abord comme césar puis comme auguste (337). En 343, Constant y fait un court séjour personnel, mais il ne semble pas être accompagné par sa famille. De 340 à 367 , Trier est abandonnée par la cour. Constance II vit surtout à Antioche, Sirmium, puis Milan, tandis que Julien demeure à Paris entre 356 et 360 . Valentinien I, sa famille et la cour se réinstallent seulement à partir de 367 . Gratien (375-383) et aussi Magnus Maximus (383-388) résident tous deux à Trier. Le dernier empereur à séjourner brièvement dans cette ville est Valentinien II en 389-390. Dans l'hypothèse où la mode des épingles à tête anthropomorphe stylisée a été lancée depuis Trier, la période qui s'accorde au mieux avec cet événement est la décennie 330-340, lorsque le jeune Constantin II y séjourne avec sa famille. Cette période concorde bien avec ce que l'on sait de la chronologie des épingles (voir supra, p. 340). En revanche, les raisons qui auraient pu conduire à privilégier une forme de représentation plastique plus gallo-romaine que romaine nous échappent.

Dans cette hypothèse, comment comprendre le processus de fabrication et de propagation? L'analyse technique des épingles et le peu de déchets de taille découverts dans les rares lieux de fabrication connus semblent indiquer que ces objets étaient façonnés en petit nombre et sans doute à la demande. Le fait que des activités de taille sont localisées dans des sites ruraux, à l'écart des agglomérations, est peut-être un indice pour évoquer le rôle d'artisans itinérants, mais il faut bien reconnaître qu'aucune preuve de leur existence ne peut être apportée. De la même façon, on aimerait savoir comment la mode des coiffures est diffusée dans les provinces et les cités. Outre les monnaies, dont le caractère édifiant a été évoqué, ce sont vraisemblablement des tableaux peints sur panneau de bois ou sur toile. qui sont utilisés. L'existence de tels supports est connue par 
les sources antiques ${ }^{23}$, comme par les découvertes archéologiques. Les portraits sur panneau de bois qui ornent les momies dites du Fayoum sont peints du vivant des personnes représentées et n'ont été intégrés dans le cartonnage des momies qu'à leur décès (Parlasca, Seemann dir., 1999, p. 40-44). L'existence de tableaux de ce type est attestée dans le monde antique et pas seulement en Égypte ni seulement dans un contexte funéraire. On peut donc penser que des tableaux analogues pouvaient véhiculer des modèles de coiffure parmi les dames des provinces.

En fin de compte, l'étude des épingles débouche sur une vision de la société provinciale gallo-romaine du nord de la Gaule, où des femmes de différents milieux s'efforcent de suivre les dernières tendances de la mode, telle qu'elle est définie par la capitale régionale ou mieux encore par la cour impériale. Il se dégage ainsi une image intéressante de la circulation de certaines modes, à une époque où le « siècle » est encore largement romain.

\section{Nota bene}

Nous tenons à remercier pour leur accueil et leur aide dans la réalisation de cette étude, $M$. Amandry (Cabinet des Médailles), L. Bakker (Römisches Museum, Augsburg), M. Barbier (conservateur départemental, Haute-Marne), I. Bardiès (musée de la Croix-d'Or, Metz), J.-C. Béal (université de Lyon II), P. Bertin (INRAP), O. Blin (INRAP),

23. Voir Daremberg, Saglio dir., 1887, art. imago, p. 393, 402, 405-406, 414-415 ; Chuvin, 1991, p. 248, citant Libanios, Progymnasmata, VIII, p. 399-401 ; par ex. aussi Pline le Jeune, $H . N$., XXXV, 147-148 ; Histoire Auguste, V. Com., XI, 9 ; V. Heligg., XXVII, 5 ; V. Alex. Sev., XIII, 2 ; V. Tyr. Trig., XXIX, 4.
P. Bordeaux (société archéologique de Touraine), L. Bourgeois (université de Poitiers), L. Buchet (CNRS, UMR 6130), P. Chardron-Picault (service archéologique municipal d'Autun), H. Chew (musée des Antiquités nationales, Saint-Germain-en-Laye), M.-H. Corbiau (service des fouilles de la région Wallonne), D. Coxall (unité d'archéologie de Saint-Denis), C. Cribellier (service régional de l'archéologie du Centre), N. Crummy (Colchester Archaeological Trust), V. Deloffre (musée archéologique, Bavay), W. Dijkman (Taakgroep kultureel Erfgoed, Dienst SDG, Maastricht), R. Durand (CNRS, UMR 7041), M. Erdrich (université de Nijmegen), S. Faust (Rheinisches Landesmuseum, Trier), M. Feugère (CNRS), K.J. Gilles (Rheinisches Landesmuseum, Trier), C. Grappin (service régional de l'archéologie de Lorraine), S. Greep (Ayr, U. K.), B. Grosjean (musée de Dijon), M. Grünewald (Museum im Andreasstift, Worms), J. Hoevenberg (Thermenmuseum, Heerlen), J. Krier (musées de l'État, Luxembourg), E. Labastie (musée archéologique de la région de Breteuil), C. Landry-Burille, F. Laubenheimer (CNRS, UMR 7041), P. Laurent (dépôt d'Escolives-SainteCamille), C. Metzger (musée du Louvre), M. Montigaud (société archéologique de Touraine), G. Moureau (société archéologique de Waremme), $\mathrm{K}$. Olson (university of Western Ontario), P. Ouzoulias (service régional de l'archéologie d'Île-de-France), D. Paillard (Conseil général du Calvados), N. Rodriguez-Meyer (unité d'archéologie de Saint-Denis), Y. Romegoux (service régional de l'archéologie du Nord - Pas-de-Calais), J.-C. Routier (INRAP), A. Thuet (INRAP), A. Vaillant (musée d'Art et d'Histoire, Langres), P. Velay (musée Carnavalet, Paris), L. Verslype (FNRS, Belgique), J. C. Wilhelm OSB (Trier) et M. Wyss (unité d'archéologie de Saint-Denis). 


\section{CATALOGUE}

\section{LISTE DES DÉCOUVERTES D’ÉPINGLES À TÊTE ANTHROPOMORPHE STYLISÉE}

Afin d'éviter les répétitions, les éléments descriptifs des épingles se réfèrent aux caractères détaillés sur la figure 11, p. 339. Le nombre de tores est indiqué par la lettre T. Sauf indication contraire, les dimensions de la tête comprennent la figure et les tores. Les lieux de découvertes sont indiqués sur la carte figure 12, p. 342.

\section{Allemagne}

Köln, Stadtkreis Köln.

1) os, RLM Köln, inv. 21283. Biblio. : inédit (renseignement S. Greep).

Krefeld-Gellep, Kreis Kempen-Krefeld : fortification, trouvailles isolées lors de la construction du port sur le Rhin. Des ébauches d'épingles à différents stades d'élaboration et des chutes de bois de cerf suggèrent la présence possible d'un atelier de tabletterie.

2) os, pas d'incision sur le front, B-2, C-3, $1 \mathrm{~T}$, complète, L. tot. : $101 \mathrm{~mm}$, L. tête : $15 \mathrm{~mm}$, collection H. Langfeld, Kempen. Biblio. : inédit (renseignement L. Bakker).

3) os, 2 incisions A-3, B-1, C-1, 2 T, L. tot. : $69 \mathrm{~mm}$, L. tête : $16 \mathrm{~mm}$, collection H. Langfeld, Kempen. Biblio. : inédit (renseignement L. Bakker).

4) os, 2 incisions A-1, B-1, C-1, 2 T, L. tot. : 88 mm, L. tête : $18,5 \mathrm{~mm}$, collection H. Langfeld, Kempen. Biblio. : inëdit (renseignement L. Bakker).

5) os, 2 incisions A-3, B-2, C-1, 2 T, L. tot. : 96 mm, L. tête : $15 \mathrm{~mm}$, collection H. Langfeld, Kempen. Biblio. : inédit (renseignement L. Bakker).

Mainz, Stadtkreis Mainz : agglomération et fortification.

6) Görzstiftung (Legionskastell) : os, 1 incision A-8, C-2, l T, brisée (tête seule), L. tête : env. $18 \mathrm{~mm}$, RGZM Mainz, inv. 024548. Biblio. : Behrens, 1917-1918, p. 33, fig. $15, n^{\circ} 14$

7) Gautor : os, 2 incisions A-3, B-1, C-1, 1 T, brisée, L. tot. : $54 \mathrm{~mm}$, L. tête: $18 \mathrm{~mm}$, Landesmuseum Mainz, inv. 0.953. Biblio. : Mikler, 1997, p. 146, $\mathrm{n}^{\circ} 35 / 7$ et pl. 35, $\mathrm{n}^{\circ} 7$.

8) Gautor: os, 1 incision A-8, face postérieure non illustrée, C-2, 1 T, complète, L. tot. : $90 \mathrm{~mm}$, L. tête : 11,5 mm, Landesmuseum Mainz, inv. 0.966. Biblio.: Mikler, 1997, p. 146, n $35 / 9$ et pl. $35, n^{\circ} 9$.

9) Gautor: os, 1 incision A-6, face postérieure non illustrée, C-2, $1 \mathrm{~T}$, complète, L. tot. : $98 \mathrm{~mm}$, L. tête : 15,5 mm, Landesmuseum Mainz, inv. 0.426. Biblio.: Mikler, 1997, p. 146, $\mathrm{n}^{\circ} 35 / 11$ et pl. 35, n० 11.

10) Ank 24.12.19: os, 2 incisions A-3, B-5, C-2, nez large et épaté, $1 \mathrm{~T}$, brisée, L. tot. : $32 \mathrm{~mm}$, L. tête : $14 \mathrm{~mm}$, Landesmuseum Mainz, inv. F.4151. Biblio. : Mikler, 1997, p. $146, \mathrm{n}^{\circ} 35 / 8$ et pl. $35, \mathrm{n}^{\circ} 8$.
11) Provenance indéterminée : os, 1 incision A-8, B-5, C-2, $1 \mathrm{~T}$, complète, L. tot. : $72 \mathrm{~mm}$, L. tête : $16 \mathrm{~mm}$, Landesmuseum Mainz, inv. F.2760b. Biblio.: Mikler, 1997 , p. $146, \mathrm{n}^{\circ} 35 / 6$ et pl. $35, \mathrm{n}^{\circ} 6$.

12) Provenance indéterminée: os, 2 incisions $A-3$, face postérieure non illustrée, $\mathrm{C}-2,1 \mathrm{~T}$, complète, L. tot. : $96 \mathrm{~mm}$, L. tête : $11 \mathrm{~mm}$, Landesmuseum Mainz, inv. 0.1058. Biblio. : Mikler, 1997, p. $146, \mathrm{n}^{\circ} 35 / 10$ et pl. $35, \mathrm{n}^{\circ} 10$.

Mehring, In der Kirchhecke, Hostertsmorgen, Kreis TrierSaarburg : villa, dans une fosse-dépotoir scellée par destruction 353-355.

13) os, 1 incision A-1, face postérieure non illustrée, C-1, 2T, complète, RLM Trier. Biblio. : inédit (renseignement K.-J. Gilles).

14) os, 2 incisions A-4, face postérieure non illustrée, C-2, $2 \mathrm{~T}$, complète, RLM Trier. Biblio. : inédit (renseignement K.-J. Gilles).

Möhn, Nierster Flur, Kreis Trier Saarburg : sanctuaire de Mars Smertulitanus et Ancamma.

15) trouvée dans JK, dans la couche d'incendie inférieure : os, 3 incisions A-3, B-5, 2 T, complète (pointe ébréchée), L. tot. : $104 \mathrm{~mm}$, L. tête : 15,5 mm, RLM Trier, inv. 15123. Biblio. : Hettner, 1901, p. 46, Nr. 1 «b».

16) trouvée dans $\mathrm{H}$ : os, RLM Trier, inv. 14853. Biblio.: Hettner, 1901, p. 46, Nr. 1 «b» ${ }^{24}$.

Monheim, Haus Bürgel, Kreis Mettman : burgus.

17) os, 2 incisions A-3, B-5, C-1, 2 T, pointe brisée, L. tot. : $72 \mathrm{~mm}$, L. tête : $12 \mathrm{~mm}$ (fig. 10, $\mathrm{n}^{\circ} 4$ ). Biblio. : Fischer, 1999.

Nettersheim, Kreis Euskirchen.

18) os, 2 incisions A-3, B-5, C-2, 2 T, L. tête : 16 mm, RLM Bonn, inv. 21283. Biblio. : inédit (renseignement S. Greep).

Pommern, Kreis Cochem-Zell.

19) os, 2 incisions A-3, B-5, C-1, $1 \mathrm{~T}, \mathrm{~L}$. tête : $12 \mathrm{~mm}, \mathrm{RLM}$ Bonn, inv. 5210. Biblio.: inédit (renseignement S. Greep).

24. Renseignement du $D^{r}$ S. Faust, RLM Trier (pas vue au musée). 
Saarbrücken, Halberg, Stadtverband Saarbrücken.

20) os, musée de Saarbrücken. Biblio. : inédit.

Traben-Trarbach, Gonzlay, Kreis Bernkastel-Wittlich : site rural, couche de destruction datée de 353 .

21) os, 2 incisions A-1, B-2, C-1, 2 T, pointe brisée, L. tot. : $66 \mathrm{~mm}$, L. tête : $18 \mathrm{~mm}$ (fig. $10, \mathrm{n}^{\circ}$ 7), Mittelmoselmuseum Traben-Trarbach. Biblio. : Binsfeld, 1973, p. 126 , fig. $3, n^{\circ} 7$.

Trier, Stadtkreis Trier : ville et faubourgs.

22) Augustinerhof (Grabung Hindenburg Realgymnasium) : os, 2 incisions horizontales sur la tempe gauche, 1 incision horizontale sur la tempe droite, B-3, 2 T, brisée, L. tot. : $57 \mathrm{~mm}$, L. tête : $15 \mathrm{~mm}$, RLM Trier, 1927, inv. 326. Biblio. : inédit.

23) Altbachtal, sanctuaire Kapelle $M:$ os, 2 incisions A-1, B-2, C-1, 1 T, extrémité de la pointe brisée, L. tot. : $97 \mathrm{~mm}$, L. tête : $11 \mathrm{~mm}$, RLM Trier, inv. ST 11923a. Biblio. : Loeschcke ed., 1942, p. 116, pl. 23, n² 26 ; Gose, 1972, p. 145, fig. 286, n 26.

24) Altbachtal, sanctuaire : os, 1 incision A-8, B-5, 2 T, brisée (conservée aux deux tiers, tige éclatée), L. tot. : $78 \mathrm{~mm}$, L. tête : $14 \mathrm{~mm}$, RLM Trier, inv. ST 12243. Biblio. : inédit.

25) Antoniusstrasse : os, 2 incisions A-1, B-1, C-1, 2 T, complète (extrémité de la pointe brisée), L. tot. : 105 mm, L. tête : 17 mm, RLM Tricr, inv. 1886, 12617. Biblio. : inédit.

26) Barbarathermen (?) : os, 2 incisions A-3, C-1, 2 T, brisée, RLM Trier, inv. 1076 ( $n^{\circ}$ erroné). Biblio. : inédit (renseignement $M$. Feugère, décrit d'après photo, objet non retrouvé au RLMT).

27) Barbarathermen : os, 3 incisions A-3, B-5, C-2, l T, complète (la pointe est raccourcie et retaillée après bris), L. tot. : $73 \mathrm{~mm}$, L. tête : $16 \mathrm{~mm}$, RLM Trier, 1882, inv. 6511. Biblio. : inédit.

28) Barbarathermen, cour est II, trouvaille août 1883, exemplaire en cours de fabrication rejeté avant achèvement (le tore inférieur n'est pas dégagé de la tige, elle-même n'ayant pas dépassé le stade d'un épannelage grossier, voir fig. 10, $\left.\mathrm{n}^{\circ} 19\right)$ : os, 2 incisions A-1, B-4, C-1, 2 T, conservée aux deux tiers, L. tot. : $74 \mathrm{~mm}$, L. tête: 17 mm, RLM Trier, 1883, inv. 8543. Biblio. : inédit.

29) Barbarathermen: os, 3 incisions A-3, B-5, C-2, 2 T, complète, L. tot. : $85 \mathrm{~mm}$, L. tête : $14 \mathrm{~mm}$, RLM Trier, 1883 , inv. 9006 . Biblio. : inédit.

30) Barbarathermen: os, 2 incisions A-2 en raison d'un défaut (éclat) sur la face gauche de la tête, B-5, C-1, 2 T, brisée, L. tot. : $76 \mathrm{~mm}$, L. tête : $16,5 \mathrm{~mm}$, RLM Trier, 1884, inv. 10457. Biblio. : inédit.

31) Barbarathermen : os, 2 incisions A-3, B-2, 2 T, complète, L. tot. : $77 \mathrm{~mm}$, L. tête : $18,5 \mathrm{~mm}$, RLM Trier, 1885 , inv.
11464. Biblio.: inédit. La pointe est raccourcie et retaillée après bris.

32) Biwer, dépôt de matériaux de construction provenant vraisemblablement de Trier : os, 3 incisions A-3, B-2, C-1, $1 \mathrm{~T}$, brisée (pointe retaillée), L. tot. : $67 \mathrm{~mm}$, L. tête : $10 \mathrm{~mm}$, RLM Trier, 1983, inv. 72a. Biblio. : inédit.

33) Biwer, dépôt de matériaux de construction provenant vraisemblablement de la Moselle à Trier : os, 3 incisions A-6, B-5, C-1, 2 T, brisée, L. tot. : $60 \mathrm{~mm}$, L. tête : $12 \mathrm{~mm}$, RLM Trier, 1983, inv. 72b. Biblio. : inédit.

34) Böhmerstrasse : os, 2 incisions A-2, B-2, 2 T, conservée aux deux tiers, L. tot. : $75 \mathrm{~mm}$, L. tête : $18 \mathrm{~mm}$, RLM Trier, 1902, inv. ST 2927a. Biblio. : inédit.

35) Böhmerstrasse : os, 2 incisions sur la tempe gauche et 3 incisions sur la tempe droite, B-2, C-2, 1 T, complète, L. tot. : $106 \mathrm{~mm}$, L. tête : 12 mm, RLM Trier, 1902, inv. ST 4273b. Biblio. : inćdit.

36) Brotstrasse : os, 2 incisions A-1, B-2, C-2, $2 \mathrm{~T}$, conservée aux deux tiers, L. tot. : $77 \mathrm{~mm}$, L. tête : $18 \mathrm{~mm}$, RLM Trier, 1902, inv. ST 3744b. Biblio. : inédit.

37) Brotstrasse (Ecke Hosenstrasse) : os, 3 incisions A-3, B-5, C-1, $2 \mathrm{~T}$, conservée aux deux tiers, L. tot. : $80 \mathrm{~mm}$, L. tête : $16 \mathrm{~mm}$, RLM Trier, 1902, inv. ST 5065. Biblio. : inédit.

38) Brotstrasse : os, 3 incisions A-3, B-5, 1 T, conservée aux deux tiers, L. tot. : $71 \mathrm{~mm}$, L. tête : $16 \mathrm{~mm}$, RLM Trier, 1902, inv. ST 5085. Biblio. : inédit.

39) Domfreihof, Domplatz : os, 3 incisions A-1, B-1, C-1, 1 T, conservée aux deux tiers, L. tot. : $60 \mathrm{~mm}$, L. tête : 14 mm, RLM Trier, 1902, inv. ST 5389. Biblio. : inédit.

40) Domfreihof, Domplatz, trouvaille en 1994: os, 2 incisions $\mathrm{A}-3, \mathrm{~B}-5$, arcades sourcilières marquées par un chevron, $1 \mathrm{~T}$, tige renflée, facture assez grossière, quasi complète (pointe brisée), L. tot. : $88 \mathrm{~mm}$; tête : $11 \mathrm{~mm}$, inv. Delta 94/200. Biblio. : Merten, 2001, p. 16, fig. 9.

41) Eberhardstrasse : os, 2 incisions A-1, B-1, C-1, 2 T, brisée, L. tot. : $60 \mathrm{~mm}$, L. tête : $20 \mathrm{~mm}$, RLM Trier, 1903, inv. ST $6804 d$. Biblio. : inédit.

42) Gilbertstrasse : os, 2 petites incisions A-2, 2 incisions sur le pan coupé, $2 \mathrm{~T}$, brisée, L. tot. : $43 \mathrm{~mm}$, RLM Trier, 1902, inv. 13. Biblio. : inédit.

43) Johannisstrasse : os, 2 incisions A-1, B-2, 2 T, brisée aux deux tiers, L. tot. : $91 \mathrm{~mm}$, L. tête : $16 \mathrm{~mm}$, RLM Trier, 1901, inv. ST 1929. Biblio. : inédit.

44) Kapellenstrasse (depuis 1969 : Saarbrücker Strasse) : os, 3 incisions A-3, B-5, C-1, 2 T, brisée, L. tot. : $56 \mathrm{~mm}$, L. tête : $13 \mathrm{~mm}$, RLM Trier, 1913, inv. 219d. Biblio.: inédit.

45) Kapellenstrasse (depuis 1969 : Saarbrücker Strasse) : os, 2 incisions A-7, B-5, C-2, 2 T, brisée aux deux tiers, L. tot. : $70 \mathrm{~mm}$, L. tête : 16,5 mm, RLM Trier, 1913, inv. $219 \mathrm{e}$. Biblio. : inédit. 
46) Maar, cimetière nord de Trier, à proximité de la Porta Nigra: os, 1 incision A-8, 3 incisions sur le pan coupé C-1, 2 T, complète, L. tot. : $68 \mathrm{~mm}$, L. tête : $16 \mathrm{~mm}$, RLM Trier, 1880, inv. 2906. Biblio. : inédit.

47) Metzelstrasse : os, 2 incisions A-1, B-2, 2 T, complète, L. tot. : $90 \mathrm{~mm}$, L. tête : $14 \mathrm{~mm}$, RLM Trier, 1902, inv. ST 4609. Biblio. : inédit.

48) Palaststrasse : os, 1 incision A-8, B-4, $2 \mathrm{~T}$, brisée aux deux tiers (la pointe est retaillće), L. tot. : $66 \mathrm{~mm}$, L. tête : 18 mm, RLM Trier, 1902, inv. ST 5928a. Biblio. : inédit.

49) Palaststrasse : os, 2 incisions A-7, B-4, C-1, 2 T, complète, L. tot. : $92 \mathrm{~mm}$, L. tête : $14 \mathrm{~mm}$, RLM Trier, 1902, inv. ST 5930. Biblio. : inédit.

50) Sans précision: os, 2 incisions A-3, B-4, C-1, 2 T, complète, L. tot. : $89 \mathrm{~mm}$, L. tête : $17,5 \mathrm{~mm}$, RLM Trier, 1938, inv. 1094a. Biblio. : inédit.

51) Sans précision: os, 2 incisions A-8, B-1, C-1, 2 T, complète (la pointe est raccourcie et retaillée après bris), L. tot. : $66 \mathrm{~mm}$, L. tête : $17 \mathrm{~mm}$, RLM Trier, 1938, inv. 1094c. Biblio. : inédit.

52) Sans précision : os, 2 incisions A-3, B-5, C-1, 2 T, brisée, L. tot. : $62 \mathrm{~mm}$, L. tête : $17 \mathrm{~mm}$, RLM Trier, 1938, inv. $1094 \mathrm{~d}$. Biblio. : inédit.

53) Trier (?) : os, 2 incisions A-3, B-5, C-1, 2 T, brisée, L.. tot. : $33 \mathrm{~mm}$, L. tête : $15 \mathrm{~mm}$, RLM Trier, 1938, inv. 1094e. Biblio. : inédit.

54) Trier (?) : os, 2 incisions A-3, B-4, C-2, 2 T, brisée, L. tot. : $58 \mathrm{~mm}$, L. tête: $17 \mathrm{~mm}$, RLM Trier, 1938, inv. $1094 \mathrm{f}$. Biblio. : inédit.

55) Trier (?) : os, 2 incisions A-3, B-4, C-1, 2 T, brisée, L. tot. : $76 \mathrm{~mm}$, L. tête : $17 \mathrm{~mm}$, RLM Trier, 1938, inv. 1094g. Biblio. : inédit.

56) Sans précision : os, 2 incisions A-1, B-4, C-1, 2 T, complète (traces de la découpe de la baguette visibles), L. tot. : $92 \mathrm{~mm}$, L. tête : $17 \mathrm{~mm}$, RLM Trier, 1938, inv. 1094h. Biblio. : inédit.

57) Sans précision : os, incisions informes sur le front et les tempes (gravure de la tête très sommaire), B-4, C-1, $2 \mathrm{~T}$, complète, L. tot. : $111,5 \mathrm{~mm}$, L. têtc : $19 \mathrm{~mm}$, RLM Trier, 1938, inv. 1094i. Biblio. : inédit.

58) Trier (?) : os, 2 incisions A-3 sur la tempe gauche et 2 incisions A-1 sur la tempe droite, B-2, C-1, $1 \mathrm{~T}$, complète, L. tot. : $104 \mathrm{~mm}$, L. tête : $12 \mathrm{~mm}$, RLM Trier, 1938, inv. 1094k. Biblio. : inédit.

59) Sankt-Matthias, fouilles 1996-1997: os, l incision latérale sur le front et les tempes, face postérieure non documentée, $\mathrm{C}-1,1 \mathrm{~T}$, brisée (pointe manquante), L. tot. : 94,5 mm, L. tête: $12 \mathrm{~mm}$, RLM Trier, inv. EV 1996,68-Fnr 167 K2. Biblio.: Kasparek, Wilhelm, 2001, p. 50.

60) Sankt-Matthias, fouilles 1996-1997 : os, 2 incisions A-6, face postérieure non documentée, $\mathrm{C}-1,1 \mathrm{~T}$, complète,
L. tot. : $103 \mathrm{~mm}$, L. tête: $11 \mathrm{~mm}$, RLM Trier, inv. EV 1996,68-Fnr 167 K1. Biblio. : Kasparek, Wilhelm, 2001, p. 50.

61) Simeonstrasse : os, 3 incisions A-3, B-5, 1 T, complète, L. tot. : $92 \mathrm{~mm}$, L. tête: $14 \mathrm{~mm}$, RLM Trier, 1903, inv. ST 6947. Biblio. : inédit.

62) Südallee : os, 2 incisions A-1, B-5, C-1, 1 T, brisée, L. tot. : $52 \mathrm{~mm}$, L. tête : 12,5 mm, RLM Trier, 1901, inv. ST 1969. Biblio. : inédit.

63) Wallstrasse (épingle de type hybride: mélange type anthropomorphe et épingle à tête féminine sur buste, voir fig. 10, $\mathrm{n}^{\circ}$ 18) : os, 2 incisions A-4, B-2, C-1, yeux marqués par deux trous ronds, tête posée sur un bustc, l T, complète, L. tot. : $130 \mathrm{~mm}$, RLM Trier, 1903, inv. ST 6248d. Biblio. : Collectif, 1983, $\mathrm{n}^{\circ} 241 \mathrm{~d}$.

64) Sans précision, trouvée vraisemblablement vers 1880 1882 : os, 3 incisions A-3, B-5, C-1, 2 T, complète, L. tot. : 112 mm, L. tête: 13 mm, RLM Trier, 1915, inv. 156. Biblio. : inédit.

65) Trier (?) : os, 1 incision A-3, B-5, C-1, 1 T, brisée, L. tot. : $69 \mathrm{~mm}$, L. tête: $12 \mathrm{~mm}$, RLM Trier, 1906, inv. 246. Biblio. : inédit.

66) Trier ou région : os, 2 incisions A-1, 2 incisions sur le pan coupé, C-1, 2 T, complète, L. tot. : $89 \mathrm{~mm}$, RLM Trier, 1880, inv. PM 3123. Biblio. : inédit.

67) Trier ou région: os, 3 incisions A-3, B-5, 1 T, brisée, L. tot. : $69,5 \mathrm{~mm}$, L. tête : $12 \mathrm{~mm}$, RLM Trier, 1912, inv. ST 9446. Biblio. : inédit.

68) Trier ou région : os, complète, L. tot. : $92 \mathrm{~mm}$, RLM Trier, sans $\mathrm{n}^{\circ}$ inventaire. Biblio. : inédit.

69) Trier ou région : os, brisée, L. tot. : $67 \mathrm{~mm}$, RLM Trier, sans $\mathrm{n}^{\circ}$ inventaire. Biblio. : inédit.

70) Trier ou région : os, brisée, L. tot. : $59 \mathrm{~mm}$, RLM Trier, sans $\mathrm{n}^{\circ}$ inventaire. Biblio. : inédit.

71) Trier ou région : os, brisée, L. tot. : $91 \mathrm{~mm}$, RLM Trier, sans $\mathrm{n}^{\circ}$ inventaire. Biblio. : inédit.

Wittlich, villa du Liesertal, Kreis Bernkastel-Wittlich : trouvaille 1904, bâtiment sud, pièce 44, dans couche d'incendie datée vers 350 . 25 .

72) os, 1 incision A-8, B-5, C-1, $1 \mathrm{~T}$, complète, L. tot. : $103 \mathrm{~mm}$, RLM Trier, 1904, inv. 252. Biblio.: Collectif, 1983, p. 278, n² 241-c ; Van Ossel, 1992, p. $279,5 \mathrm{c}$.

73) os, 3 incisions A-3, B-4, C-1, 2 T, complète, L. tot. : $103 \mathrm{~mm}$, L. tête : $14 \mathrm{~mm}$, RLM Trier, 1904, inv. 253. Biblio. : inédit.

74) os, 1 incision A-8, B-2, $1 \mathrm{~T}$, brisée aux deux tiers, très petite tête, L. tot.: $72 \mathrm{~mm}$, L. tête: $11 \mathrm{~mm}$, RLM Trier, 1904, inv. 254. Biblio.: Van Ossel, 1992, p. 279,5 c.

25. D'après I.. Hussong, Trierer Zeitschrift, 16/17, 1941-1942, p. 325. 
Worms, Kreis Worms : provenance et contexte inconnus.

75) os, 1 incision A-8, B-2, C-1, 1 T, brisée aux deux tiers, L. tot. : $65 \mathrm{~mm}$; L. tête : $14 \mathrm{~mm}$ (fig. 10, $\mathrm{n}^{\circ} 17$ ), Museum der Stadt (Worms), inv. R 3617 A. Biblio. : inédit (renseignement S. Greep et M. Grünewald).

\section{BELgiQue}

Arlon (musée), province de Luxembourg: provenance indéterminée.

76) os, 3 incisions A-3, C-1, 2 T, brisée, pointe retaillée, gravure très plate, musée archéologique d'Arlon. Biblio. : citée par Robert, 1976.

77) os, 3 incisions A-3, C-l, $1 \mathrm{~T}$, complète, gravure très creuse, tête fine et très petite, musée archéologique d'Arlon. Biblio. : citée par Robert, 1976 ; cette épinglc est vraisemblablement celle dc Wolkrange-Sesselich (selon Robert, 1976, p. 123).

78) os, 1 incision A-8, C-1, $2 \mathrm{~T}$, complète, musée archéologique d'Arlon. Biblio. : citée par Robert, 1976.

Ave-et-Auffe, Génimont, province de Namur : site rural.

79) os, 2 incisions A-3, B-5, C-1, 2 T, conservée aux deux tiers, L. tot. : $62 \mathrm{~mm}$, L. tête : $16 \mathrm{~mm}$. Biblio. : Plumier, Dupont, 1995, p. 87 , fig. $4, \mathrm{n}^{\circ} 4$.

Braives, Les Sarrasins, province de Liège: agglomération, secteur central (BI-19).

80) os, 2 incisions sur le front et les tempes, B-2, C-1, 2 T, complète, L. tot. : $79 \mathrm{~mm}$, inv. 77580. Biblio. : Massart, 1981, p. 108-109, fig. $45, \mathrm{n}^{\circ} 15$

Dourbes, La Roche à Lomme, province de Namur : fortification de hauteur.

81) os, 2 incisions, A-7, B-5, C-1, 2 T, tête seule conservée, les yeux seraient marqués par deux petits trous, L. tot. : 16,5 mm. Biblio. : Robert, 1976, p. 121, a.

82) os, 2 incisions A-3, B-2, C-1, 2 T, tête seule conservée, L. tot. : $12 \mathrm{~mm}$. Biblio. : Robert, 1976, p. 121, b.

83) os, 2 incisions A-1, B-2, C-1, 2 T, tête seule conservée, L. tot. : 13 mm. Biblio. : Robert, 1976, p. 121, c.

84) os, 2 incisions A-1, B-2, C-1, 2 T, tête seule conservée, fendue, L. tot. : $13 \mathrm{~mm}$. Biblio.: Robert, 1976, p. 121, d.

85) os, 2 incisions A-1 sur les tempes, B-5, C-1, 2 T, tête seule conservée, L. tot. : $10 \mathrm{~mm}$. Biblio. : Robert, 1976 , p. 123, e.

86) os, brisée (fragment de tête), collection privée R. Hottiaux (Bruxelles). Biblio. : Robert, 1976, p. 123, f (simple signalement).

87) os, A-1, B-2, tête seule conservée, collection privée J. François de Lausprelle (Acoz). Biblio. : Robert, 1976, p. 123, g (simple signalement).
Furfooz, Hauterecenne, province de Namur: fortification de hauteur, hors stratigraphie.

88) os, 3 incisions A-3, C-1, 2 T, brisée, L. tot. : $40 \mathrm{~mm}$. Biblio. : Brulet, 1978, p. 72, fig. $46, \mathrm{n}^{\circ} 9$.

Hainaut (province) : provenance indéterminée.

89) os, 3 incisions en chevrons parallèles, B-2, complète, L. tot. : $104 \mathrm{~mm}$, L. tête: $12 \mathrm{~mm}$, musée de Mons. Biblio. : Robert, 1976, p. 123.

Tongres (musée), province de Limburg : provenance indéterminée.

90) os, 3 incisions A-5, 3 incisions B-4, C-1, 2 T, complète, L. tot. : $110 \mathrm{~mm}$, L. tête : $19 \mathrm{~mm}$, Provinciaal GalloRomeins Museum (Tongeren), inv. E 22. Biblio.: Daniëls, 2001, § $1.14 \mathrm{n}^{\circ} 193$, pl. VII $\mathrm{n}^{\circ} 193$.

Tournai, Saint-Pierre, province de Hainaut : contexte urbain, prov. 207.

91) os, 2 incisions A-3, B-3, C-1, 2 T, pointe brisée, L. tot. : 72 mm, inv. 742. Biblio. : Brulet, Verslype, 1999, p. 118, fig. $64, \mathrm{n}^{\circ} 15$ et p. $121, \mathrm{n}^{\circ} 15$.

Wolkrange-Sesselich, Breden Felder, Sabel Feld, Staffeld, province de Luxembourg : villa détruite vers 353.

92) os, 3 incisions A-3, B-5, C-1, 1 T, complète, L. tot. : $82 \mathrm{~mm}$, L. tête : $9 \mathrm{~mm}$. Biblio. : Halbardier, Rausch, 1969, p. 43, fig. épingle $n^{\circ} 3$; Rohert, 1976, p. 123.

\section{France}

Alisc-Sainte-Reine, Côte-d'Or : Croix-Saint-Charles ou La Fanderolle, agglomération, fouilles Espérandieu (1909-1914).

93) os, 2 incisions A-3, B-4, C-1, 2 T, brisée, L. tot. : 77,8 mm, L. tête : $17 \mathrm{~mm}, \mathrm{MAN}$, inv. 10526. Biblio. : inédit (renseignement C. Grappin).

Amiens, Somme : ZAC cathédrale, église Saint Firmin.

94) os, inv. 1994 S2. 1048-1. Biblio. : Bernard, Celval, 1994, pl. non paginée.

Argonne (?) : provenance inconnue, collection G. Chenet.

95) os, 2 incisions A-3, B-5, C-1, 2 T, incomplète, L. tot. : $26 \mathrm{~mm}$, L. tête : $15 \mathrm{~mm}, \mathrm{MAN}$, inv. 83572. Biblio. : inédit.

Arras, Pas-de-Calais : rue Baudimont, casernes théodosiennes (390-410).

96) os, $A-4$, face postérieure non illustrée, $\mathrm{C}-1,2 \mathrm{~T}$, incomplète (pointe brisée), L. tot. : $90 \mathrm{~mm}$, inv. E 88-W.90-91. Biblio. : Jacques, 1990 , p. $91, \mathrm{n}^{\circ} 570$.

Autun, Saône-et-Loire : lieux de découverte inconnus.

97) os, A-1, B-5, 4, C-1, 2 T, brisée, L. tot. : 58 mm, L. tête : 
23 mm, musée Rolin. Biblio.: Collectif, 1978, p. 34, $\mathrm{n}^{\circ} 116$ et pl. IX, $\mathrm{n}^{\circ} 10$; Béal, 1987, p. 200, n³79a.

98) os, A-1, B-5, C-1, 2 T, brisée, L. tot. : $54 \mathrm{~mm}$, L. tête : 24 mm, musée Rolin. Biblio. : Béal, 1987, p. 200, n 379b.

99) os, A-3, B-5, C-1, 2 T, brisée, L. tot. : $70 \mathrm{~mm}$, L. tête : 22 mm, musée Rolin. Biblio. : Béal, 1987, p. 200, n 379c.

100) os, A-1, B-4, C-1, 2 T, brisée, L. tot. : $53 \mathrm{~mm}$, L. tête : 19 mm, musée Rolin. Biblio. : Béal, 1987, p. 200, n 379d.

101) os, A-3, B-4, C-1, 2 T, complète, pointe retaillée, L. tot. : $57 \mathrm{~mm}$, L. tête : $15 \mathrm{~mm}$, musée Rolin. Biblio. : Béal, 1987, p. $200, \mathrm{n}^{\circ} 379 \mathrm{e}$.

102) os, A-3, face postérieure non illustrée, C-1, 2 T, complète, L. tot. : $87 \mathrm{~mm}$, L. tête : $15 \mathrm{~mm}$, musée Rolin. Biblio. : Collectif, 1978, p. $34, \mathrm{n}^{\circ} 116$ et pl. IX ; Béal, 1987, p. 200, n³ 379f.

103) os, A-2, face postérieure non illustrée, C-1, $2 \mathrm{~T}$, complète, L. tot. : $75 \mathrm{~mm}$, L. tête : $19 \mathrm{~mm}$, musée Rolin. Biblio. : Béal, 1987, p. 200, n³79g.

104) os, A-3, B-2, C-1, 2 T (le supérieur est tronconique), conservée aux deux tiers, L. tot. : $73 \mathrm{~mm}$, L. tête : 15 mm, musée Rolin. Biblio. : Béal, 1987, p. 200, n 379h.

105) os, A-3, B-2 ; C-1, 2 T, complète, L. tot. : 70 mm, L. tête : 12 mm, musée Rolin. Biblio. : Béal, 1987, p. 200, n 379i.

106) os, non illustrée, complète, pointe retaillée, L. tot. : $73 \mathrm{~mm}$, L. tête : $14 \mathrm{~mm}$, musée Rolin. Biblio. : Béal, 1987, p. $200, \mathrm{n}^{\circ} 379 \mathrm{j}$.

107) os, A-3, face postérieure non illustrée, C-1, 2 T, brisée, L. tot. : $65 \mathrm{~mm}$, L. tête : $15 \mathrm{~mm}$, musée Rolin. Biblio. : Béal, 1987, p. $200, \mathrm{n}^{\circ} 379 \mathrm{k}$.

108) os, A-3, face postérieure non illustrée, C-1, 2 T, complète, L. tot. : $92 \mathrm{~mm}$, L. tête : $19 \mathrm{~mm}$, musée Rolin. Biblio. : Béal, 1987, p. 200, n $379 \mathrm{l}$.

109) os, A-2, B-2, C-1, $1 \mathrm{~T}$, conservée aux deux tiers, pointe retaillée, L. tot. : $60 \mathrm{~mm}$, L. tête : $14 \mathrm{~mm}$, musée Rolin. Biblio. : Collectif, 1978, p. $34, \mathrm{n}^{\circ} 116$ et pl. IX ; Béal, 1987 , p. $200, \mathrm{n}^{\circ} 379 \mathrm{~m}$.

110) os, A-3, B-5, C-1, 2 T (dont l'un est mal façonné), brisée, L. tot. : $76 \mathrm{~mm}$, L. tête : $18 \mathrm{~mm}$, musée Rolin. Biblio. : Béal, 1987, p. 200, n³79n.

111) os, A-3, face postérieure non illustrée, C-1, $2 \mathrm{~T}$, complète, L. tot. : $83 \mathrm{~mm}$, L. tête : $18 \mathrm{~mm}$, musée Rolin. Biblio. : Béal, 1987, p. 200, n³79o.

112) os, A-3, B-5, C-1, 2 T, conservée aux deux tiers, L. tot. : $41 \mathrm{~mm}$, L. tête : $15 \mathrm{~mm}$, musée Rolin. Biblio. : Béal, 1987, p. $200, \mathrm{n}^{\circ} 379 \mathrm{p}$.

Auxerre, Yonne : Sainte-Nitasse, villa.

113) os, 1 incision A-8, B-4, C-1, $2 \mathrm{~T}$, complète, L. tot. : $107 \mathrm{~mm}$, L. tête : $11 \mathrm{~mm}$, dépôt SRA Dijon. Biblio. : inédit.

Bavay, Nord : ZAP ou inconnu, contexte urbain.

114) os, 3 incisions A-4, B-5 (?), C-2 (?), 2 T, complète, L. tot. :
$97 \mathrm{~mm}$, L. tête : $17 \mathrm{~mm}$, musée de Bavay, inv. Y 437 (non retrouvée au musée). Biblio.: Carmelez, 1982, p. 111, pl. V, $\mathrm{n}^{\circ} 7$; un exemplaire est cité aussi par Robert, 1976, p. 124.

115) os, 2 incisions A-4, B-2, C-l, $2 \mathrm{~T}$, brisée, L. tot. : $48 \mathrm{~mm}$, L. tête : $16 \mathrm{~mm}$, musée de Bavay, inv. Z4005. Biblio. : Carmelez, 1982, p. 111, pl. V, $\mathrm{n}^{\circ} 8$; un exemplaire est cité aussi par Robert, 1976, p. 124.

116) os, 3 incisions A-2, B-5, C-1, complète (la pointe a sans doute été retaillée), $2 \mathrm{~T}$, L. tot. : $72 \mathrm{~mm}$, L. tête : $17 \mathrm{~mm}$, musée de Bavay, inv. 4Z69. Biblio.: Carmelez, 1982, p. 111, pl. V, $n^{\circ} 9$ (dessin incomplet); un exemplaire est cité aussi par Robert, 1976, p. 124.

117) os, 3 incisions A-4, B-3, C-1, 2 T, brisée, L. tot. : $61 \mathrm{~mm}$, L. tête: $17 \mathrm{~mm}$, musée de Bavay, inv. Z2892. Biblio. : inćdit.

118) os, 2 incisions A-1, B-2, C-1, 2 T, complète, L. tot. : $95 \mathrm{~mm}$, L. tête : $14 \mathrm{~mm}$, musée de Bavay, inv. 657486 . Biblio. : inédit.

119) os, 2 incisions A-1, B-2, C-1, 2 T, complète, L. tot. : $101 \mathrm{~mm}$, L. tête : $17 \mathrm{~mm}$, musée de Bavay, sans $\mathrm{n}^{\circ}$ inv. Biblio. : inédit.

Bennecourt, Yvelines : La Butte du Moulin à Vent, sanctuaire, bâtiment $\mathrm{A}$, US 6005 (démolition intérieure, avec des monnaies allant jusqu'au début des années 380), US 6019 et US 6053 (enrochement du mur ouest).

120) os, 1 incision A-8, B-3, C-1, 2 T, brisée (conservée aux deux tiers), L. tot. : $48 \mathrm{~mm}$, L. tête : $11 \mathrm{~mm}$, dépôt SADY, inv. 35-6053. Biblio. : Bourgeois dir., 1999, p. 121 et fig. $91, n^{\circ} 719$.

121) os, 2 incisions A-1, B-2, C-1, 2 T, brisée, brûléc, L. tot. : $28 \mathrm{~mm}$, L. tête : $18 \mathrm{~mm}$, dépôt SADY, inv. 35-6053. Biblio. : Bourgeois dir., 1999, p. 121 et fig. $91, \mathrm{n}^{\circ} 720$.

122) os, 2 incisions A-1, B-2, C-3, 2 T, complète, L. tot. : $85 \mathrm{~mm}$, L. tête : $19 \mathrm{~mm}$ (fig. $10, \mathrm{n}^{\circ} 5$ ), dépôt SADY, inv. 35-6005. Biblio.: Bourgeois dir., 1999, p. 121 et fig. $91, \mathrm{n}^{\mathrm{c}} 721$.

123) os, 2 incisions A-6, B-1, C-1, 2 T, brisée, L. tot. : $52 \mathrm{~mm}$, L. tête : $19 \mathrm{~mm}$ (fig. 10, $\mathrm{n}^{\circ}$ 2), dépôt SADY, inv. 35-6005. Biblio. : Bourgeois dir., 1999, p. 121 et fig. $91, \mathrm{n}^{\circ} 722$.

124) os, 1 incision A-8, B-3, C-1, 2 T, brisée, L. tot. : $35 \mathrm{~mm}$, L. tête: $13 \mathrm{~mm}$, dépôt SADY, inv. 35-6019. Biblio. : Bourgeois dir., 1999, p. 121 et fig. $91, \mathrm{n}^{\circ} 718$.

Bliesbruck, Moselle : Steinfelder, Im Sand, ensemble thermal.

125) os, 3 incisions A-3, face postérieure non illustrée, C-1, 2 T, complète. Biblio. : Petit, Schaub, 1995, p. 50, photo.

Bourges, Cher : Saint-Martin-des-Champs, nécropole antique, dans une tombe d'immature, sous la tête.

126) os, A-7 ? (2 incisions à g., 1 incision à dr.), B-1, C-2, 2 T, extrémité de la tige brisée, L. tot. : $86 \mathrm{~mm}$, L. tête : 
$21 \mathrm{~mm}$, inv. Fl2-41 (12101)/1448. Biblio. : inédit (renseignement R. Durand).

Chartres, Eure-et-Loir: parvis de la cathédrale, contexte urbain.

127) os, 2 incisions A-1, face postérieure non illustrée, $\mathrm{C}-1$, 1 T, complète, L. tot. : $82 \mathrm{~mm}$, L. tête : 16,5 mm. Biblio. : Randoin et al., 1995, p. 39.

Châteaubleau, Seine-et-Marne : agglomération et sanctuaire.

128) La Tannerie (sanctuaire de source), associée à des monnaies de 335-341 : os, 2 incisions A-1, pas d'incisions sur le pan coupé, $\mathrm{C}-1,2 \mathrm{~T}$ à peine esquissés, tête $\mathrm{s}$ eule, L. tot. : $19 \mathrm{~mm}$, inv. CH.81.01.11. Biblio. : Collectif, 1982 , p. 24 ; Binet, 1994 , p. 112 et p. 116, pl. XLIV, $\mathrm{n}^{\circ} 3$.

129) L'Aumône (grand sanctuaire), dans le remblai du puits, dans une couche datée par des monnaies du premier quart du IV s. : os, 2 incisions A-4, B-5, C-1, 2 T, complète, L. tot. : $97 \mathrm{~mm}$, L. tête : $15 \mathrm{~mm}$, inv. CH.90.VI.25.1066.01. Biblio. : Binet, 1994, p. 112 et p. 116 , pl. XLIV, $n^{\circ} 4$.

130) L'Aumône (grand sanctuaire), secteur $X$, gisement 21 : os, A-1, B-2, C-1, 2 T, conservée aux deux tiers, L. tot. : $62 \mathrm{~mm}$, L. tête : $14 \mathrm{~mm}$, inv. CH.75.04.02. Biblio. : Binet, 1994, p. 112 et p. 116, pl. XLIV, $\mathrm{n}^{\circ} 1$.

131) Théâtre (parcelle ZA 31), secteur XI, gisement 4, fouilles 1968-1989: os, 2 incisions A-3, B-2, C-1, 2 T, brisée, L. tot. : $21 \mathrm{~mm}$, L. tête : $17 \mathrm{~mm}$, inv. CH.79.02.04. Biblio. : Binet, 1994, p. 112 et p. 116, pl. XLIV, n 2.

Compans, Seine-et-Marne : l'ouest du Parc, villa, seconde moitié du IVes.

132) fosse 001 : os, 2 incisions A-1, B-1, C-2, arête du nez non marquée, $2 \mathrm{~T}$, complète, L. tot. : $102 \mathrm{~mm}$, L. tête : $17 \mathrm{~mm}$ (fig. 10, $\mathrm{n}^{\circ} 13$ ), dépôt JPGF Le Bourget, inv. 182.01.225. Biblio. : inédit.

133) fosse 001 : os, 2 incisions A-1, B-2, C-3, 2 T, pointe brisée, L. tot. : $74 \mathrm{~mm}$, L. tête : $19,5 \mathrm{~mm}$, dépôt JPGF Le Bourget, inv. 182.01.226. Biblio. : inédit.

134) fosse 001 : os, 2 incisions A-1, B-1, C-1, 2 T, brisée, tête seule, L. tot. : $34 \mathrm{~mm}$, L. tête : 19,5 mm, dépôt JPGF Le Bourget, inv. 182.01.230. Biblio. : inédit.

135) fosse 001 : os, 2 incisions A-1, B-1, C-1, 2 T, pointe brisée, L. tot. : $79 \mathrm{~mm}$, L. tête : $17 \mathrm{~mm}$, dépôt JPGF Le Bourget, inv. 182.01.231. Biblio. : inédit.

136) fosse 001 : os, 2 incisions A-1, B-3, C-1, 2 T, pointe brisée, L. tot. : $70 \mathrm{~mm}$, L. tête : $14 \mathrm{~mm}$, dépôt JPGF Le Bourget, inv. 182.01.232. Biblio. : inédit.

137) fosse 077 : os, 2 incisions A-1, B-1, C-1, 2 T, complète, pointe retaillée, L. tot. : $70 \mathrm{~mm}$, L. tête : $23 \mathrm{~mm}$ (fig. 10 , $\mathrm{n}^{\circ}$ 12), dépôt JPGF Le Bourget, inv. 182.077.005. Biblio. : inédit.
Congy, Marne : trouvaille funéraire ?, sépulture 17 ? (1908 ?).

138) os, 3 incisions A-1 sur le cou en raison de la petite taille de la tête et du front, B-5, C-1, 2 T, complète, L. tot. : $81 \mathrm{~mm}$, L. tête : 13,5 mm, MAN, inv. 81094.j (ancicnnc collection Cottel). Biblio. : inédit.

Dambron, Eure-et-Loir : fosse Dieppe, site rural, vers 335-340/ 350-355.

139) os, 1 incision A-8, B-1, C-3, base du nez soulignée par une incision horizontale, $2 \mathrm{~T}$, brisée, conservée aux deux tiers. Biblio.: Ferdière, 1979, p. 47, fig. 25 et fig. 26,1 .

Épiais-Rhus, Val-d'Oise : La Poulaine, villa.

140) os, 2 incisions A-8, B-4, C-1, 2 T, conservée aux deux tiers, L. tot. : $57 \mathrm{~mm}$, musée de Guiry, inv. E13. Biblio. : Joy, 1989 , p. 80-81, dessin 1.

141) os, 1 incision A-3, B-5, C-1, 2 T, complète, L. tot. : 76 mm, musée de Guiry, inv. WH8. Biblio. : Joy, 1989, p. 80-81, dessin 2.

142) os, 2 incisions A-1, B-2, C-1, 2 T, brisée, L. tot. : $76 \mathrm{~mm}$, musćc de Guiry, inv. WE5. Biblio. : Joy, 1989, p. 80-81, dessin 3.

Épiais-Rhus, Val-d'Oise : agglomération.

143) théâtre : os, musée de Guiry. Biblio. : inédit (renseignement L. Bourgeois).

144) villa de l'Éros: os, musée de Guiry. Biblio.: inédit. (renseignement L. Bourgeois).

145) villa de l'Éros: os, musée de Guiry. Biblio.: inédit (renseignement L. Bourgeois).

146) villa de l'Éros: os, musée de Guiry. Biblio.: inédit (renseignement L. Bourgeois).

Escolives-Sainte-Camille, Yonne : Grippe Soleil, villa.

147) os, 1 incision A-8, B-2, C-3, 2 T, brisée, L. tot. : $73 \mathrm{~mm}$, L. tête : $16 \mathrm{~mm}$, dépôt d'Escolives-Sainte-Camille, inv. E.415. Biblio. : inédit.

148) os, A-1, B-2, C-1, 2 T, brisée (tête seule), L. tot. : 24 mm, L. tête : $19 \mathrm{~mm}$, dépôt d'Escolives-Sainte-Camille, inv. E.413. Biblio. : inédit.

149) os, A-1, B-1, C-1, 1 T, brisée (tête seule), L. tot. : $16 \mathrm{~mm}$, dépôt d'Escolives-Sainte-Camille, inv. E.403. Biblio. : inédit.

150) os, A-4, B-4, C-1, 2 T, complète, L. tot. : $81 \mathrm{~mm}$, L. tête : $16 \mathrm{~mm}$, dépôt d'Escolives-Sainte-Camille, inv. E.402. Biblio. : inédit.

151) os, A-4, B-5, C-1 , 1 T, brisée (tête seule), L. tot. : 18 mm, dépôt d'Escolives-Sainte-Camille, sans $\mathrm{n}^{\circ}$ inv. Biblio.: Prost, 1981 , p. $11-45$; 1983, p. $263-299$ ( $\left.{ }^{\circ} 208\right)$; 1989, p. $210, \mathrm{n}^{\circ} 293$.

152) os, A-4, B-2, C-1, 2 T, brisée (tête seule), L. tot. : 22 mm, L. tête : $18 \mathrm{~mm}$, dépôt d'Escolives-Sainte-Camille, 
inv. E.99. Biblio. : Prost, 1981, p. 11-45 ; 1983, p. $263-299$ $\left(\mathrm{n}^{\circ} 207\right) ; 1989$, p. $210, \mathrm{n}^{\circ} 293$.

153) os, A-1, B-3, C-1, $1 \mathrm{~T}$, brisée (tête seule), L. tot. : $31 \mathrm{~mm}$, L. tête : $20 \mathrm{~mm}$, dépôt d'Escolives-Sainte-Camille, inv. E.73. Biblio. : Prost, 1981, p. 11-45 ; 1983, p. 263-299 (n॰206); 1989, p. 210, n²93.

154) os, A-1, B-2, C-1, 2 T, complète, L. tot. : 77 mm, L. tête : $14 \mathrm{~mm}$, dépôt d'Escolives-Sainte-Camille, inv. E.64 ESC68CDT8. Biblio.: Prost, 1981, p. 11-45; 1983, p. 263-299 (n $\left.{ }^{\circ} 205\right) ; 1989$, p. $210, \mathrm{n}^{\circ} 293$.

155) os, A-l, pas d'incisions sur le pan coupé, $C-1$, pas de $T$, complète, L. tot. : $82 \mathrm{~mm}$, L. tête : $19 \mathrm{~mm}$, dépôt d'Escolives-Sainte-Camille, inv. E.77 ESC61PDC47. Biblio. : Prost, 1981, p. 11-45 ; 1983, p. 263-299 (n² 204) ; 1989 , p. $210, \mathrm{n}^{\circ} 293$.

156) os, A-1, B-1, C-1, 2 T, complète, L. tot. : $103 \mathrm{~mm}$, L. tête : $17 \mathrm{~mm}$, dépôt d'Escolives-Sainte-Camille, inv. E.96 ESC71GS157. Biblio.: Prost, 1981, p. 11-45; 1983, p. 263-299 (n² 203); 1989, p. $210, \mathrm{n}^{\circ} 293$.

$157)$ os, A-4, B-2, C-1, 2 T, complète, L. tot. : $108 \mathrm{~mm}$, L. tête : $15 \mathrm{~mm}$, dépôt d'Escolives-Sainte-Camille, inv. E.88 ESC71GS80. Biblio. : Prost, 1981, p. 11-45; 1983, p. 263-299 (n²02); 1989, p. $210, \mathrm{n}^{\circ} 293$.

Évreux, Eure.

$158)$ os. Biblio. : inédit (renseignement F. Laubenheimer).

159) os. Biblio. : inédit (renseignement $\mathrm{F}$. Laubenheimer).

Forêt de Compiègne, Oise : fouilles indéterminées de A. de Roucy pour Napoléon III, vers 1860-1870. En raison de leur état et de leur aspect de surface, il est possible que plusieurs de ces épingles proviennent de sépultures.

160) os, 2 incisions A-6, B-2, C-1, 2 T, complète, L. tot. : $95 \mathrm{~mm}$; L. tête : 16,5 mm, MAN, inv. 29175.e. Biblio. : inédit.

161) La Carrière du Roi (?) : os, 2 incisions A-1, B-4, C-1, 2 T, complète, L. tot. : $95 \mathrm{~mm}$, L. tête : $16 \mathrm{~mm}, \mathrm{MAN}$, inv. 29175.a. Biblio. : inédit.

162) os, 3 incisions A-3, B-5, C-1, 1 T, brisée, L. tot. : $63,5 \mathrm{~mm}$, L. tête : $17 \mathrm{~mm}, \mathrm{MAN}$, inv. 13746. Biblio. : inédit.

163) os, 3 incisions A-1 peu marquées, B-2, C-1, complète, L. tot. : $98,5 \mathrm{~mm}$, L. tête : $12 \mathrm{~mm}, \mathrm{MAN}$, inv. 14287. Biblio. : inédit.

164) os, 2 incisions A-2, B-4, C-2, 2 T, complète, L. tot. : $98,5 \mathrm{~mm}$, L. tête : $14 \mathrm{~mm}, \mathrm{MAN}$, inv. 14267. Biblio. : inédit.

165) os, 2 incisions A-4, pan coupé abîmé, C-1, 2 T, complète, L. tot. : $92 \mathrm{~mm}$, L. tête : $16 \mathrm{~mm}, \mathrm{MAN}$, inv. 29175. Biblio. : inédit.

166) os, 2 incisions A-3, B-5, C-1, 2 T, complète, L. tot. : $97 \mathrm{~mm}$, L. tête : $15 \mathrm{~mm}, \mathrm{MAN}$, inv. 29175. Biblio. : inédit.

167) os, 2 incisions A-3, pan coupé abîmé, sans doute B-4,
C-1, 2 T, brisée, L. tot. : $79 \mathrm{~mm}, \mathrm{~L}$. tête : $16 \mathrm{~mm}, \mathrm{MAN}$, inv. 29175. Biblio. : inédit.

168) os, 2 incisions A-1 sur les tempes, B-2, C-1, 2 T, complète, L. tot. : 94 mm, L. tête : 13 mm, MAN, inv. 29175. Biblio. : inédit.

169) os, 2 incisions A-4, pan coupé abîmé, sans doute B-5, C-1, 2 T, complète, L. tot. : $92 \mathrm{~mm}$, L. tête : $17 \mathrm{~mm}$, MAN, inv. 29175. Biblio. : inédit.

170) os, 2 incisions A-1, pan coupé abîmé, sans doute B-2, C-1, 2 T, complète, L. tot. : $92 \mathrm{~mm}$, L. tête : $14 \mathrm{~mm}$, MAN, inv. 29175. Biblio. : inédit.

171) os, 2 incisions A-1, B-2, C-1, $2 \mathrm{~T}$, complète, L. tot. : 90,5 mm, L. tête: $14 \mathrm{~mm}$, MAN, inv. 29175. Biblio. : inédit.

172) os, front et tempes abîmés, pan coupé abîmé, C-1, 2 T, complète, L. tot. : $85,5 \mathrm{~mm}$, L. tête : $14 \mathrm{~mm}, \mathrm{MAN}$, inv. 29175. Biblio. : inédit.

173) os, 2 incisions A-1, B-1, C-2, $1 \mathrm{~T}$, brisée, L. tot. : $68 \mathrm{~mm}$, L. tête : 7 mm, MAN, inv. 29175. Biblio. : inédit.

174) os, 2 incisions A-1, B-2, C-1, 2 T, pointe brisée, L. tot. : $80 \mathrm{~mm}$, L. tête: $14,5 \mathrm{~mm}$, MAN, inv. 29175. Biblio. : inédit.

175) os, 2 incisions A-4, B-5, C-1, 2 T, complète (pointe brisée), L. tot. : $92 \mathrm{~mm}$, L. tête : $12 \mathrm{~mm}$, MAN, inv. 29175. Biblio. : inédit.

Grenoble, Isère : basilique Saint-Iaurent, niveau de circulation daté du $\mathbf{I V}^{\mathrm{c}}$ s. dans la zone d'inhumations située au sud de l'église funéraire.

176) os, 2 incisions A-3, B-5, C-1, $2 \mathrm{~T}$, brisée, L. tot. : $31 \mathrm{~mm}$, L. tête: 19 mm, inv. 672/1323. Biblio.: Burille, 1996 ; Colardelle, 1999.

Jouars-Pontchartrain, Yvelines: La Ferme d'Ithe, agglomération, contexte $1388, V^{e}$ s.

177) os, 2 incisions A-3, B-3, C-1, $2 \mathrm{~T}$, brisée (tête seule), L. tête : 23 mm, inv. 3498-1. Biblio. : inédit.

La Croix-Saint-Ouen, Oise : Mont Chyprès, fouilles 1870.

178) os, 3 incisions A-1, B-1, C-1, 2 T, complète (extrémité de la pointe brisée), L. tot. : $92 \mathrm{~mm}$, L. tête : $7 \mathrm{~mm}, \mathrm{MAN}$, inv, 14293.b. Biblio. : inédit.

179) os, 2 incisions A-3, B-2, C-1, 1 T, complète, L. tot. : $84,5 \mathrm{~mm}$, L. tête : $12 \mathrm{~mm}, \mathrm{MAN}$, inv. 29153. Biblio. : inédit.

Langres, Haute-Marne : contexte urbain, sans précision.

180) os, 3 incisions A-3, B-6, C-1, 2 T, tige renflée, L. tot. : $78 \mathrm{~mm}$, L. tête : $12 \mathrm{~mm}$, musée d'Art et d'Histoire (Langres), inv. n 912-72. Biblio. : Béal, 1987, n 379. 
Limé, Aisne : Pont d'Ancy, sépulture $n^{\circ} 205$ (?). Ces épingles ${ }^{26}$ font partie d'un lot comprenant 5 épingles en argent, 50 en os et 25 en bronze, dont certaines à tête polyédrique et à tête en pomme de pin.

181) os, 2 incisions A-3, B-3, C-1, 2 T, complète, L. tot. : $89 \mathrm{~mm}$, L. tête : $11,5 \mathrm{~mm}, \mathrm{MAN}$, inv. 36759. Biblio. : Moreau, 1887, pl. 64 et 75 .

182) os, 2 incisions A-1, B-1, C-1, 2 T, complète, L. tot. : $94 \mathrm{~mm}$, L. tête: $13 \mathrm{~mm}, \mathrm{MAN}$, inv. 36759. Biblio. : Moreau, 1887, pl. 64 et 75.

183) os, 2 incisions A- 1 sur tempe droite et 2 incisions A-4 sur tempe gauche, B-2, C-1, 2 T, complète, L. tot. : $95 \mathrm{~mm}$, L. tête : 13 mm, MAN, inv. 36517. Biblio. : Moreau, 1887, pl. 64 et 75 .

184) os, 2 incisions A-1, B-1, C-2 (face abîmée), 2 T, pointe brisée, L. tot. : $85 \mathrm{~mm}$, L. tête : $15,5 \mathrm{~mm}$, MAN, inv. 36517. Biblio. : Moreau, 1887, pl. 64 et 75.

185) os, 2 incisions A-3, B-2, C-1, 2 T, complète, L. tot. : 100,5 mm, L. tête : 16 mm, MAN, inv. 36517. Biblio. : Moreau, 1887, pl. 64 et 75 .

186) os, 2 incisions A-1 sur le cou, B-5, C-2, 2 T, brisée, L. tot. : $69 \mathrm{~mm}$, L. tête: $11 \mathrm{~mm}, \mathrm{MAN}$, inv. 36517. Biblio. : Moreau, 1887, pl. 64 et 75 .

187) os, 2 incisions A-4, B-2, C-1, 2 T, complète, L. tot. : $96 \mathrm{~mm}$, L. tête: $15 \mathrm{~mm}, \mathrm{MAN}$, inv. 36517. Biblio. : Moreau, 1887, pl. 64 et 75.

188) os, 2 incisions A-3, B-2, C-1, 2 T, complète, L. tot. : $97 \mathrm{~mm}, \mathrm{~L}$. tête : 13,5 mm, MAN, inv. 36517. Biblio. : Moreau, 1887, pl. 64 et 75 .

189) os, 2 incisions A-3, B-3, C-1, 2 T, complète, L. tot. : 95,5 mm, L. tête: $13 \mathrm{~mm}, \mathrm{MAN}$, inv. 36517. Biblio. : Moreau, 1887, pl. 64 et 75 .

190) os, 2 incisions A-3, B-3, C-1, 2 T, complète, L. tot. : 94,5 mm, L. tête: $13 \mathrm{~mm}, \mathrm{MAN}$, inv. 36517. Biblio. : Moreau, 1887, pl. 64 et 75 .

191) os, 3 incisions A-3, B-5, C-1, 2 T, complète, L. tot. : $100 \mathrm{~mm}$, L. tête: $14 \mathrm{~mm}$, MAN, inv. 36517. Biblio. : Moreau, 1887, pl. 64 et 75 .

192) os, 2 incisions A-3, B-1, face abîmée, 2 T, brisée, L. tot. : $54 \mathrm{~mm}$, L. tête: $13 \mathrm{~mm}, \mathrm{MAN}$, inv. 36517. Biblio.: Moreau, 1887 , pl. 64 et 75.

Limetz-Villez, Yvelines : La Bosse Marnière, villa.

193) cabane Str. 3, US 9006 (milieu ou troisième quart du $\mathrm{IV}^{\mathrm{c}} \mathrm{s}$.) : os, 2 incisions $\mathrm{A}-1, \mathrm{~B}-1, \mathrm{C}-1,2 \mathrm{~T}$, complète,

26. La plupart des épingles de Limé ont des dimensions très proches, en particulier les têtes mesurent presque toutes $13 \mathrm{~mm}$ environ. Par ailleurs, les caractères typologiques sont très similaires. L'impression d'un travail en série s'impose, malgré les physionomies différentes. Les dessins publiés dans l'Album Caranda ne présentent que fort peu de points communs avec les pièces conservées au musée des Antiquités nationales de Saint Germain-en-Laye, au point qu'il cst difficilc dc fairc coincider un dessin avec une épingle particulière.
L. tot. : 102,5 mm, L. tête : $24 \mathrm{~mm}$ (fig. $10, \mathrm{n}^{2} 6$ ), inv. LBM/86/9006/D2. Biblio. : Van Ossel, Ouzoulias, 1987 , p. 14, fig. $12, \mathrm{n}^{\circ} 2$.

194) bâtiment principal, US $1007:$ os, 3 incisions A-1 sur les tempes, B-5, C-1, 2 T, brisée, L. tot. : $70 \mathrm{~mm}$, L. tête : $15 \mathrm{~mm}$, inv. LBM/83-8/1007/2. Biblio. : inédit.

195) US 13013: os, 2 incisions A-3, B-2, C-2, 2 T, brisée, L. tot. : $19 \mathrm{~mm}$, L. tête : $14 \mathrm{~mm}$, inv. LBM/ 83-8/13013/17. Biblio. : inédit.

Lisieux, Calvados: collège Michelet, sépulture $n^{\circ} 370$, deuxième quart du $\mathrm{IV}^{\mathrm{c}} \mathrm{s}$.

196) os, 3 incisions A-1, B-2, C-1, 2 T, complète, L. tot. : $87 \mathrm{~mm}$, L. tête : $17,2 \mathrm{~mm}$ (fig. 10, $\mathrm{n}^{\circ} 8$ ). Biblio. : Collectif, 1994, p. 74, $\mathrm{n}^{\circ} 125,1$ (renseignements D. Paillard).

197) os, 2 incisions A-1, B-2, C-1, 2 T, complète, L. tot. : $92 \mathrm{~mm}$, L. tête : 14,3 mm (fig. 10, $\mathrm{n}^{\circ}$ 9). Biblio. : Collectif, 1994, p. 74, $\mathrm{n}^{\circ} 125,2$ (renseignements D. Paillard).

198) os, 2 incisions A-1, B-2, C-1, 2 T, complète, L. tot. : $91 \mathrm{~mm}$, L. tête : $14,7 \mathrm{~mm}$ (fig. $10, \mathrm{n}^{\circ} 10$ ). Biblio. : Collectif, 1994, p. 74, $\mathrm{n}^{\circ} 125,3$ (renseignements D. Paillard).

Meaux, Seine-et-Marne : La Bauve, sanctuaire suburbain.

199) os, 2 incisions A-1, face postérieure non illustrée, C-1, 2 T. Biblio. : Collectif, 1998, p. 107 (à gauche).

200) os, 2 incisions A-1, face postérieure non illustrée, C-1, 2 T. Biblio. : Collectif, 1998, p. 107 (à droite).

Meaux, Seine-et-Marne: palais épiscopal, contexte urbain, caves du palais, niveau de la seconde moitié du IV $\mathrm{s}$. L'épingle était associée avec un peigne type Böhme B.

201) os, A-3, B-5, C-2, $1 \mathrm{~T}$, complète, L. tot. : $92 \mathrm{~mm}$, musée Bossuet (Meaux), inv. 982-13-26. Biblio.: Collectif, $1984 a$, p. $163, n^{\circ} 497$ et pl. IV, $n^{\circ} 497$.

Messy, Seine-et-Marne : La Mare au Roi, habitat rural.

202) os, 2 incisions A-6, B-2, C-3, 2 T, complète, L. tot. : $65 \mathrm{~mm}$, L. tête : $18 \mathrm{~mm}$. Biblio. : inédit (renseignement P. Bertin, INRAP).

Metz, Moselle : rue du Chanoine-Collin, près des murs I, III et IV.

203) os, 2 T, Biblio. : Biehler, Schlemaire, 1974, p. 11 et fig. 13.

204) os, 2 T, complète. Biblio.: Biehler, Schlemaire, 1974, p. 11 et fig. 13.

205) os, 2 T, complète. Biblio. : Biehler, Schlemaire, 1974, p. 11 et fig. 13 .

206) os, 1 T, complète. Biblio. : Biehler, Schlemaire, 1974, p. 11 et fig. 13. 
Metz, Moselle : musée, ancienne collection Huber, sans provenance.

207) os, 2 incisions A-3, B-5, C-1, 2 T, complète, L. tot. : $80 \mathrm{~mm}$, L. tête : $18 \mathrm{~mm}$, musée de Metz, inv. 3393. Biblio. : Collectif, 1981, p. XXVI, $\mathrm{n}^{\circ} 75$.

208) os, 2 incisions A-1, B-1, C-1, 2 T, pointe brisée, L. tot. : $69 \mathrm{~mm}$, musée de Metz, inv. 3394. Biblio. : Collectif, 1981, p. XXVI, $\mathrm{n}^{\circ} 75$.

209) os, 1 incision A-8, B-4, C-1, 2 T, brisée, L. tot. : $45 \mathrm{~mm}$, L. tête: 17,5 mm, musée de Metz, inv. 3654. Biblio. : Collectif, 1981, p. XXVI, $\mathrm{n}^{\circ} 75$.

Montmartin, Oise : Le Moulin à Vent, villa. Trouvée avec une épingle à tête en pomme de pin.

210) os, 1 incision A-8, face postérieure non illustrée, C-3, base du nez marquée par une incision, $2 \mathrm{~T}$, brisée (conservée aux deux tiers), L. tot. : $83 \mathrm{~mm}$, L. tête : $18 \mathrm{~mm}$. Biblio. : Saint-Blanquat, 1992, p. 175.

Noyon, Oise : contexte urbain.

211) os, 3 incisions A-2 (haute coiffure), face postérieure non représentée, C-3, 2 T. Biblio. : Arbogast et al., 1987, p. 76 (à gauche).

212) os, 2 incisions A-3, face postérieure non représentée, C-1, 2 T. Biblio. : Arbogast et al., 1987, p. 76 (au milieu).

213) os, 1 incision A-3, face postérieure non représentée, $C-1$, 2 T. Biblio. : Arbogast et al., 1987, p. 76 (à droite).

Orléans, Loiret.

214) os, $1 \mathrm{~T}$, conservée aux deux tiers, L. : $73 \mathrm{~mm}$, musée d'Orléans, inv. 0.15/3433. Biblio. : Collectif, 1989a, p. 88.

215) os, $2 \mathrm{~T}$, tête seule, musée d'Orléans, inv. 0.15/1068. Biblio. : Collectif, 1989a, p. 88.

Paris : fonds ancien du musée Carnavalet.

216) os, A-3, B-2, C-1, 1 T, brisée, L. tot. : $83 \mathrm{~mm}$, L. tête : $14 \mathrm{~mm}$, musée Carnavalet, inv. AY 45/13. Biblio.: Collectif, 1990 ; Dureuil, Béal, 1996, p. 42.

217) os, A-6, B-2, C-1, 1 T, brisée (conservée aux deux tiers), L. tot. : $66 \mathrm{~mm}$, L. tête : $14 \mathrm{~mm}$, musée Carnavalet, inv. AY 48/28. Biblio. : Collectif, 1990 ; Dureuil, Béal, 1996 , p. 42.

218) os, A-1, B-1, C-1, 2 T (conservée aux deux tiers), L. tot. : $83 \mathrm{~mm}$, L. tête : $27 \mathrm{~mm}$, musée Carnavalet, inv. YB 27/3. Biblio. : Collectif, 1990 ; Dureuil, Béal, 1996, p. 42.

219) os, A-1, face postérieure non illustrée, C-1, $2 \mathrm{~T}$, complète, L. tot. : $88 \mathrm{~mm}$, L. tête : $22 \mathrm{~mm}$, musée Carnavalet. Biblio. : Collectif, 1990 ; Dureuil, Béal, 1996, p. 43.

220) os, A-3, face postérieure non illustrée, $\mathrm{C}-1,2 \mathrm{~T}$, brisée (tête seule), L. tot. : $29 \mathrm{~mm}$, L. tête : $18 \mathrm{~mm}$, musée Carnavalet. Biblio. : Collectif, 1990 ; Dureuil, Béal, 1996, p. 42.
221) os, $2 \mathrm{~T}$, complète, L. tot. : $65 \mathrm{~mm}$, L. tête : $12 \mathrm{~mm}$, musée Carnavalet. Biblio. : Collectif, 1990 ; Dureuil, Béal, 1996, p. 42.

222) os, A-1, face postérieure non illustrée, C-1, 2 I, complète, L. tot. : $65 \mathrm{~mm}$, L. tête: $11 \mathrm{~mm}$, musée Carnavalet. Biblio. : Collectif, 1990 ; Dureuil, Béal, 1996, p. 42.

Paris : rue de Lutèce.

223) os, A-I, B-l, C-1, 2 T, complète, L. tot. : $80 \mathrm{~mm}$, L. tête : 17 mm, musée Carnavalet, inv. Lu 2057/16025. Biblio. : Collectif, 1990 ; Dureuil, Béal, 1996, p. 43.

224) os, A-3, face postérieure non illustrée, C-1, 2 T, incomplète, L. tot. : $28 \mathrm{~mm}$, musée Carnavalet, inv. LU.2162/8583. Biblio.: Dureuil, 1994, p. 69, n 26 ; Dureuil, Béal, 1996, p. 42.

225) os, faces antérieure et postérieure très abîmées, $2 \mathrm{~T}$, extrémité brisée, L. tot. : $64 \mathrm{~mm}$, musée Carnavalet, inv. LU.2192/1048. Biblio. : Dureuil, 1994, p. 69, $\mathrm{n}^{\circ} 26$; Dureuil, Béal, 1996, p. 42.

226) os, A-1, face postérieure non illustrée, C-1, $2 \mathrm{~T}$. complète, L. tot.: $83 \mathrm{~mm}$, musée Carnavalet, inv. LU.2023/16003. Biblio. : Dureuil, 1994, p. 69, $\mathrm{n}^{\circ} 26$; Dureuil, Béal, 1996, p. 42.

Pithiviers-le-Vieil, Loiret : La Grande Raye, agglomération.

227) os, 1 incision A-8, face postérieure non illustrée, C-3, 2 T, brisée (tête seule). Biblio.: Ferdière, 1985, p. 353, fig. 54b.

Pithiviers-le-Vieil, Loiret: Les Oulches, agglomération, US 5065, comblement de la cave Str. 8 (milieu du IV ${ }^{\mathrm{c}} \mathrm{s}$.). Indices vraisemblables d'un atelier de taille sur le site (caves Str. 2 et 6 : bois de cerf, andouillers sciés, baguettes taillées).

228) os, 1 incision A-8, face postérieure non illustrée, pas d'arcades sourcilières, deux profondes incisions pour les yeux, pas de nez, $1 \mathrm{~T}$, complète, $\mathrm{L}$. tête : $8 \mathrm{~mm}$ (fig. 10, ${ }^{\circ}$ 16). Biblio. : Cribellier, 1993, p. 75-76, fig. 55 et 56 (à droite).

229) os, 2 incisions A-3, face postérieure non illustrée, C-1, 2 T, L. tête : $10 \mathrm{~mm}$. Biblio. : Cribellier, 1993, p. 76, fig. 56 (à gauche).

Reims, Marne.

230) tombe avec mobilier du début du IV s., épingle trouvée en association avec 43 monnaies de Constance I, Constantin I et Constant I : os, 2 incisions sur le front et les tempes, 2 incisions sur le pan coupé, complète, L. tot. : $90 \mathrm{~mm}$. Biblio. : Petitjean, 1960, p. 18-19 et fig. 5 ; Robert, 1976, p. 124.

231) rue du Barbâtre ou avenue de Paris : os, A-3, B-3, C-1, 2 T, complète, L. tête. : 19,4 mm, musée Saint-Rémy, col- 
lection Gillet, inv. $\mathrm{n}^{\circ}$ 3-858. Biblio. : Gillet, 1928 ; Guillaume, 1970, p. 76 et fig. 24 ; Robert, 1976, p. 124.

232) rue Pluche, (emplacement du forum) : os, 2 incisions A-3, B-5, C-1, 1 T, complète, L. tot. : $73 \mathrm{~mm}$, L. tête : $15 \mathrm{~mm}$. Biblio. : Christophe, Ertlé, 1969-1970, p. 22-23 et fig. $4, \mathrm{n}^{\circ} 5$.

233) rue Boulingrin : os, 2 incisions $\mathrm{A}-3, \mathrm{~B}-2, \mathrm{C}-1$, au moins 1 T, complète. Biblio. : Erclé, 1968, p. 15, fig. 5.

Rigny-la-Salle, Meuse : villa.

234) os, 2 incisions A-1, face postérieure non illustrée, C-1, 2 T, extrémité brisée, L. tête : $16 \mathrm{~mm}$. Biblio. : Genot, 1972 , p. 55 , fig. 5 ; Ferdière dir., 1983, p. 47, fig. $26, \mathrm{n}^{\circ} 2$.

Rouen, Seine-Maritime : cour des Maçons, contexte urbain, US 4692 (fin du $I V^{c}$ s.-premier quart du $V^{e}$ s.).

235) os, 2 incisions A-1, B-5, C-2, 2 T, brisćc (conscrvéc aux deux tiers), L. tot. : $55 \mathrm{~mm}$, L. tête : $17 \mathrm{~mm}$, dépôt SRA. Biblio. : inédit.

Saint-Denis, Seine-Saint-Denis : basilique, aire 17.

236) os, 2 incisions A-6, B-1, C-1, 2 T, complète, L. tot. : $112 \mathrm{~mm}$, L. tête : $17 \mathrm{~mm}$, dépôt unité archéologique, inv. 17718/2. Biblio. : supra texte, p. 330 , fig. $8, \mathrm{n}^{\circ} 2$.

237) os, 2 incisions A-6, B-1, G-1, 2 T, brisée, L. tot. : $51 \mathrm{~mm}$, L. tête : $17 \mathrm{~mm}$, dépôt unité archéologique, inv. 17718/8. Biblio. : supra texte, p. 330 , fig. $8, \mathrm{n}^{\circ} 3$.

238) os, 2 incisions A-1, B 1, G 1, 2 T, brisée, L. tot. : $16 \mathrm{~mm}$, L. tête : $19 \mathrm{~mm}$, dépôt unité archéologique, inv. 17719/13. Biblio. : supra texte, p. 332 , fig. $8, \mathrm{n}^{\circ} 4$.

239) os, 2 incisions A-4, B-1, C-1, 2 T, brisée, L. tot. : $51 \mathrm{~mm}$, L. tête : $20 \mathrm{~mm}$, dépôt unité archéologique, inv. 172702/3. Biblio. : supra texte, p. 332 , fig. $8, \mathrm{n}^{\circ} 1$.

240) os, 5 incisions A-2, B-1, C-3, 3 T, brisée (tête seule), L. tot. : $30 \mathrm{~mm}$, L. tête : $29 \mathrm{~mm}$, dépôt unité archéologique, inv. 17978/3. Biblio. : supra texte, p. 333 , fig. 8 , $n^{\circ} 5$.

Saint-Germain-Laxis, Seine-et-Marne: Le Climat des Terres Noires, villa, fosse, fin du III $\mathrm{e}$.

241) os, A-7, B-5, C-2, $2 \mathrm{~T}$, brisée (conservée aux deux tiers), L. tot. : $85 \mathrm{~mm}$, L. tête : $16 \mathrm{~mm}$ (fig. $10, \mathrm{n}^{\circ} 1$ ). Biblio. : Séguier, 1994, p. 181 et pl. LXVIII, nº 2.

Saint-Romain, Côte-d'Or : Le Verger, site rural, III'-IV $s$.

242) os, A-7 surmontée d'une incision horizontale, face postérieure non illustrée, C-1, $2 \mathrm{~T}$, brisée (conservée aux deux tiers), L. tot. : $68 \mathrm{~mm}$, L. tête : $19 \mathrm{~mm}$. Biblio. : Collectif, s.d., pl. $16, \mathrm{n}^{\circ} 8$.

Sens, Yonne : archevêché, contexte urbain, seconde moitié du IV $\mathrm{e}$.-début du Ve $\mathrm{s}$.

243) os, A-1/A-4, B-1, C-2, $2 \mathrm{~T}$, complète, L. tot. : $116 \mathrm{~mm}$,
L. tête : 22 mm, musée de Sens, inv. K25,1. Biblio. : inédit (renseignement M. Barbier).

244) os, 2 incisions A-2, B-3, C-1, 2 T, complète (pointe retaillee), $\mathrm{L}$. tot. : $98 \mathrm{~mm}$, L. tête : $16 \mathrm{~mm}$, musêe de Sens, inv. L24,1. Biblio.: inédit (renseignement M. Barbier).

245) os, 2 incisions A-6, B-2, C-1, 2 T, incomplète, L. tot. : $40 \mathrm{~mm}$, L. tête : $18 \mathrm{~mm}$, musée de Sens, inv. 123. Biblio. : inédit (renseignement $Y$. Roumegoux).

246) os, 2 incisions A-6, B-2, C-1, 2 T, pointe brisée, L. tot. : $85 \mathrm{~mm}$, L. tête : $16 \mathrm{~mm}$, musée de Sens, inv. 132. Biblio. : inédit (renseignement $Y$. Roumegoux)

247) os, 2 incisions obliques A-2, pan coupé lisse, arcades sourcilières à peine ébauchées, $1 \mathrm{~T}$ à peine ébauché, brisée, L. tot. : $78 \mathrm{~mm}$, L. tête : $21 \mathrm{~mm}$ (fig. 10, $\mathrm{n}^{\circ} 20$ ), musée de Sens, inv. 138. Biblio. : inédit (renseignement Y. Roumegoux).

248) os, ébauche, face non traitée, pan coupé lisse, $T$ non dégagés, brisée, L. tot. : $72 \mathrm{~mm}$ (fig. 10, $\mathrm{n}^{\circ}$ 21), musée de Sens, inv. 131. Biblio. : inédit (renseignement Y. Roumegoux).

Tours, Indre-et-Loire : place Jean-Jaurès, fouilles du palais de Justice, hors contexte.

249) os, 3 incisions A-7, B-4, C-1, 2 T, brisée, inv. 1.138.0.4. Biblio. : Collectif, 1977, p. 13, n ${ }^{\circ} 40$ et fig. ; Ferdière dir., 1983, addenda et corrigenda; Motteau, 1991, p. 13, 17 et $21, n^{\circ} 44$

Tours, Indre-et-Loire : sans contexte connu.

250) os, incisions A-7 encadrant deux incisions horizontales, B-5, C-1, 2 T, brisée (tête seule), L. tot. : $23 \mathrm{~mm}$, L. tête : $16 \mathrm{~mm}$, musée de l'hôtel Gouin à Tours, inv. HG.200254-1. Biblio.: inédit (communication Société archéologique de Touraine)

Vallangoujard, Val-d'Oise : La Garenne, villa.

251) os, 1 incision A-3, face postérieure non illustrée, C-2, $2 \mathrm{~T}$, musée de Guiry-en-Vexin. Biblio.: Deboissy, 1965; Ferdière dir., 1983, p. 47, fig. 26, n 3 ; Mitard, 1999, p. $74-75$ et fig. $n^{\circ} 7$.

Vendeuil-Caply, Oise : agglomération.

252) os, 2 incisions A-3, B-1, C-1, 2 T, complète, L. tot.: $100 \mathrm{~mm}$, L. tête : $20 \mathrm{~mm}$, musée archéologique de Breteuil, inv. D 113. Biblio. : inédit (renseignement E. Labastie).

Vert-Saint-Denis, Seine-et-Marne : Les Fourneaux, villa, F 6421 , couche de démolition du petit bâtiment bordant le mur de séparation interne de la cour, datée de la fin $d u I^{c} \mathrm{~s}$. ou du début du $V^{\mathrm{c}}$ s. par molette UJC. 49 (?) sur sigillée. d'Argonne. 
253) os, 2 incisions A-6, B-1, C-3, 2 T, brisée (pointe manquante), I. tot. : $73 \mathrm{~mm}$, L. tête : $20 \mathrm{~mm}$, inv. 6421 857. Biblio. : Daveau, Goustard, 1995, p. $39^{\text {bis }}$.

Vienne, Isère : temple de Cybèle, sanctuaire.

254) os, 2 incisions A-1, B-5, C-2, 2 T, complète, L. tot. : $94,3 \mathrm{~mm}$, L. tête: $16 \mathrm{~mm}$, musée de Vienne. Biblio. : Pelletier, 1966, p. 134, fig. 31 ; Collectif, 1978, $n^{\circ} 327$, pl. XLVII, $\mathrm{n}^{\circ} 2$; Ferdière dir., 1983, p. 47, fig. $26, \mathrm{n}^{\circ} 5$; Béal, 1984, p. 26, fig. 55.

Zouafques, Pas-de-Calais : Wolphus, villa, cave comblée entre $346-350$ et 370 , couche Ib $\left(n^{\circ} 246\right)$ et sol $2\left(n^{\circ} 247\right)$ (Routier, 1990) (= couches 10 et 4 dans Thuillier, Routier, 1998, p. 5-6).

255) os, 2 incisions A-4, B-5, pas d'arcades sourcilières, $1 \mathrm{~T}$, complète, L. tot. : $88,5 \mathrm{~mm}$, L. tête : $12 \mathrm{~mm}$. Biblio. : Routier, 1990, pl. 42 ; Routier, à paraître, fig. 49B, $n^{\circ} 9$.

256) os, 1 incision A-1, face postérieure non illustrée, C-2, 2 T, incomplète, L. tot. : $15 \mathrm{~mm}$, L. tête : $14 \mathrm{~mm}$. Biblio. : Routier, à paraître, fig. $49 \mathrm{~B}, \mathrm{n}^{\circ} 10$.

Provenance inconnuc.

257) os, 1 incision A-8, B-2, C-1, $1 \mathrm{~T}$, brisée, pointe retaillée, L. tot. : $68 \mathrm{~mm}$, L. tête: $13,5 \mathrm{~mm}, \mathrm{MAN}$, inv. 86876 (ancienne collection Corbeil, 1975). Biblio. : inédit.

\section{GraND-DUChÉ DE LUXEMbouRG}

Dalheim : agglomération.

258) fond de la cave 3, détruite vers 353 : os, 2 incisions sur le pan coupé, 2 incisions sur le front et les tempes, $2 \mathrm{~T}$, brisée, L. tot. : $58 \mathrm{~mm}$, L. tête : $17 \mathrm{~mm}$, musée de Luxembourg, inv. 1978-100/121. Biblio. : Hémecht, 1978, p. 371 ; Publications de la section historique de l'Institut grand-ducal du Luxembourg, 9, 1853, p. 127 et pl. VI, $\mathrm{n}^{\circ} 31$.

259) os, 3 incisions sur le pan coupé, 2 incisions sur le front et les tempes, $1 \mathrm{~T}, \mathrm{~L}$. tot. : $81 \mathrm{~mm}, \mathrm{~L}$. tête : $15 \mathrm{~mm}$, musée de Luxembourg. Biblio. : inédit.

260) os, 2 incisions sur le pan coupé, 2 incisions sur le front et les tempes, $1 \mathrm{~T}$, tête seule, L. tête : $12 \mathrm{~mm}$, musée de Luxembourg. Biblio. : inédit.

261) os, 2 incisions sur le pan coupé, 2 incisions sur le front et les tempes, $1 \mathrm{~T}$, pointe brisée, L. tot. : $63 \mathrm{~mm}$, L. tête : $10 \mathrm{~mm}$, musée de Luxembourg. Biblio. : inédit.

262) os, 3 incisions sur le pan coupé, 3 incisions sur le front et les tempes, $1 \mathrm{~T}$, pointe brisée, L. tot. : $73 \mathrm{~mm}$, L. tête : $9 \mathrm{~mm}$, musée de Luxembourg. Biblio. : inédit.

263) os, 2 incisions sur le pan coupé, 3 incisions sur le front et les tempes, 2 T, complète, L. tot. : $106 \mathrm{~mm}$, L. tête : $17 \mathrm{~mm}$, musée de Luxembourg. Biblio. : inédit.

264) os, 2 incisions sur le pan coupé, 3 incisions sur le front et les tempes, $2 \mathrm{~T}$, complète, L. tot. : $90 \mathrm{~mm}, \mathrm{~L}$. tête : $14 \mathrm{~mm}$, musée de Luxembourg. Biblio. : inédit.

265) os, 3 incisions sur le pan coupé, 3 incisions sur le front et les tempes, $1 \mathrm{~T}$, cou souligné par un lëger creux, pointe brisée, L. tot. : $76 \mathrm{~mm}$, L. tête : $11 \mathrm{~mm}$, musée de Luxembourg. Biblio. : inédit.

266) os, 2 incisions sur le pan coupé, 3 incisions sur le front et les tempes, $1 \mathrm{~T}$, pointe brisée et retaillée, taille fruste, L. tot. : $66 \mathrm{~mm}$, L. tête : $12 \mathrm{~mm}$, musée de Luxembourg. Biblio. : inédit.

267) os, 2 incisions sur le pan coupé, 3 incisions sur le front et les tempes, $1 \mathrm{~T}$, cou souligné par un léger creux, taille fruste, pointe brisée, L. tot. : $97 \mathrm{~mm}$, L. tête : $12 \mathrm{~mm}$, musée de Luxembourg, inv. 1900-2/1354. Biblio. : inédit.

268) os, 2 incisions horizontales sur le pan coupé, $l$ incision sur le front et les tempes, $2 \mathrm{~T}$, pointe brisée, L. tot. : $90 \mathrm{~mm}$, L. tête : $15 \mathrm{~mm}$, musée de Luxembourg, inv. 1900-2/1015. Biblio. : inédit.

269) os, 3 incisions sur le pan coupé, 3 incisions sur le front et les tempes, $2 \mathrm{~T}$, pointe brisée, L. tot. : $85 \mathrm{~mm}$, L. tête : $12 \mathrm{~mm}$, musée de Luxembourg, inv. 1900-2/1014. Biblio. : inédit.

270) os, 2 incisions sur le pan coupé, 1 incision sur le front et les tempes, $1 \mathrm{~T}$, tige brisée, L. tot. : $63 \mathrm{~mm}$, L. tête : 12 mm, musée de Luxembourg, inv. 1900-2/1013. Biblio. : inédit.

271) os, 3 incisions sur le pan coupé, 3 incisions sur le front et les tempes, $1 \mathrm{~T}$, puinte brisée, L. tơl. : $77 \mathrm{~mm}$, L. tête : $13 \mathrm{~mm}$, musée de Luxembourg, inv. 1900-2/1355. Biblio. : inédit.

272) os, 3 incisions sur le pan coupé, 3 incisions sur le front et les tempes, $1 \mathrm{~T}$, tige brisée, L. tot. : $56 \mathrm{~mm}$, L. tête : $11 \mathrm{~mm}$, musée de Luxembourg, inv. 1900-2/1012. Biblio. : inédit.

273) os, 2 incisions horizontales sur le front, B-2, 2 T, brisée (tête seule), L. tot. : $25,5 \mathrm{~mm}$, L. tête: $22,5 \mathrm{~mm}$, L. d'après inventaire du musée : $75 \mathrm{~mm}$, RLM Trier, inv. 15748. Biblio. : inédit.

Diekirch : village, villa, fouilles Waringo des anncxcs (1992), occupation surtout du IV $\mathbf{s}$.

274) os. Biblio. : trouvaille inédite présentée lors de la journée archéologique luxembourgeoise de Saint-Hubert du 30.1.1993.

Givenich (Mompach) : Op den Léieren, villa.

275) bronze, 4 incisions sur le pan coupé, 2 incisions sur le front et les tempes, $1 \mathrm{~T}$, complète, tige tordue, L. tête : 14 mm, musée de Luxembourg, inv. 1983-59. Biblio.: inédit.

Steinsel : sanctuaire.

276) os, 3 incisions sur le pan coupé, 3 incisions sur le front 
et les tempes, $2 \mathrm{~T}$, complète, L. tot. : $90 \mathrm{~mm}$, L. tête : 17 mm, musée de Luxembourg, inv. D 24. Biblio. : inédit.

Titelberg : agglomération.

277) os, collection P. Kremer (Esch-sur-Alzette). Biblio. : inédit, dessin conservé au musée de Luxembourg.

Wasserbillig : rive droite de la Moselle, à $400 \mathrm{~m}$ du pont, site de nature indéterminée.

278) os, 3 incisions sur le pan coupé, 2 incisions sur le front et les tempes, $1 \mathrm{~T}$, complète, extrémité pointe ébréchée, L. tot. : $97 \mathrm{~mm}$, L. tête : $12 \mathrm{~mm}$, musée de Luxembourg, inv. 1983-154. Biblio. : inédit.

\section{Grande-BretaGne}

Canterbury, Kent : Marlowe Car Park, contexte urbain, période 4II, vers 375-450. Trouvé dans room 7 : silt in hypocaust.

279) os, A-3 (3 incisions à gauche, 1 incision à droite), B-5, C-1, 1 T, complète, L. tot. : 77,1 mm, L. tête : $10 \mathrm{~mm}$. Biblio. : Blockley et al., 1995, p. 1134, n 933 et fig. 498, $n^{\circ} 933$.

Colchester, Essex : contexte urbain.

280) os, 2 incisions A-1, B-2, C-1, 2 T, complète, L. tot. : $70 \mathrm{~mm}$, L. tête : $16,5 \mathrm{~mm}$, Colchester Museum. Biblio. : Greep, 1982, fig. 257, $\mathrm{n}^{\circ} 676$; signalée aussi dans Blockley et al., 1995, p. 1134.

Richborough, Kent : Roman fort, fortification.

Le nombre exact d'épingles trouvées dans ce site et leur identification précise continuent de soulever des difficultés, en raison des différences dans les dessins publiés. La liste ci-après a été établie d'après les informations fournies par S. Greep.

281) top soil: os, 2 incisions A-2, face postérieure non illustrée, C-2, 2 T, complète, L. tot. : 104,5 mm, L. tête : $18 \mathrm{~mm}$. Biblio. : Bushe-Fox, 1928, p. 45, pl. XIX, $\mathrm{n}^{\circ} 22$; Greep, 1982, fig. 258, $\mathrm{n}^{\circ} 681$; signalée aussi par Riha, 1990, p. 99, note 427 et par Dureuil, Béal, 1996, p. 17.

282) small find context $\mathrm{n}^{\circ} 464$, vers $280-400$ : os, 2 incisions A-3 à gauche, 2 incisions A-1 à droite, B-2, C-2 (?), 2 T, L. tête : 16,5 mm. Biblio. : Greep, 1982, fig. 257, $\mathrm{n}^{\circ} 679$; signalée par Blockley et al., 1995, p. 1134 ; Stutzinger, 1995, p. 188, note 125.

283) small find context $\mathrm{n}^{\circ} 1313$, vers 340 et plus tard: os, 1 incision A-4 sur chaque tempe, face postérieure non illustrée, Cr1 (?), 2 T, L. tête : $15 \mathrm{~mm}$. Biblio. : Greep, 1982, fig. 258, $\mathrm{n}^{\circ} 680$; signalée par Blockley et al., 1995, p. 1134 ; Stutzinger, 1995, p. 188, note 125.

284) os, 2 incisions A-8, C-2 (?), $2 \mathrm{~T}$, tige brisée aux deux ticrs (l'appartenance de cette épingle au type à tête anthropomorphe stylisée est vraisemblable, mais le dessin est manifestement interprété et amélioré, gommant l'aspect stylisé de la figuration). Biblio. : Roach Smith, 1850 , p. 85.

Southwark (London): King's Head Yard IV, contexte urbain, avec céramique du dernier quart $d u \mathrm{I}^{\mathrm{er}} \mathrm{s}$. au troisième quart du II's.

285) os, 2 incisions A-2, B-5, C-1, 2 T, brisée, L. tot. : $41 \mathrm{~mm}$, L. tête : 16,5 mm. Biblio. : Kenyon, 1959, p. 102, fig. 31 .

Ware, Hertfordshire.

286) os, 2 incisions $A-3$, face arrière non illustrée, $\mathrm{C}-1,1 \mathrm{~T}$, L. cons. : $70 \mathrm{~mm}$, L. tête : 10,5 mm. Biblio. : Greep, 1982, fig. $257, \mathrm{n}^{\circ} 678$; signalée aussi dans Blockley et al., 1995, p. 1134 .

Winchester, Hampshire (IV $\mathrm{s}$.).

287) os. Biblio. : inédit (renseignement S. Greep).

Yorkshire Caves : découverte ancienne (XIXes.).

288) os, 2 incisions A-1, B-2, C-1, 2 T, British Museum. Biblio. : Greep, 1982, fig. 257, $\mathrm{n}^{\circ} 677$.

\section{Pays-Bas}

Heerlen, Provincie Limburg : agglomération secondaire (renseignement $D^{r} \mathrm{~J}$. Hoevenberg, Thermenmuseum) ${ }^{27}$.

289) thermes (Coriovallumstraat) : os, 1 incision A-3, B-5, $\mathrm{C}-1,3$ incisions sur les faces latérales, $1 \mathrm{~T}$, complète, L. tot. : $88 \mathrm{~mm}$, L. tête : $11 \mathrm{~mm}$, Thermenmuseum, inv. 10308. Biblio. : inédit.

290) Heerlen (sans précisions) : os, 3 incisions sur les faces latérales, B-5, C-1, $1 \mathrm{~T}$, complète, L. tot. : $104 \mathrm{~mm}$, L. tête: $16 \mathrm{~mm}$, Thermenmuseum, inv. 08080. Biblio. : inédit.

Maastricht, Provincie Limburg : Pandhof, contexte urbain avec plusieurs monnaies, entre autre un aes 4 de Magnus Maximus (387-388).

291) os, pas d'incision sur le front ou les tempes, face postérieure lisse, $\mathrm{C}-1$, pas de $\mathrm{T}$ sous la tête, conservée aux deux tiers (pointe brisée), L. tot.: $76 \mathrm{~mm}$, dépôt GOBM, inv. 1996.MAVP.9/2-8-20. Biblio. : Dijkman, Ervynck, 1998, p. 38-39, fig. 25, $\mathrm{n}^{\circ} 15$.

27. Une troisième épingle à tête anthropomorphe stylisée a été trouvée en 1982 dans la palestre ouest des thermes de Heerlen (Thermenmuseum, $n^{\circ}$ inv. 10283). La coiffure représentée, comportant une résille couvrant tout le front et les tempes, appartient manifestement à un autre type. Son appartenance au type étudié dans cet article n'est pas assurée. 


\section{SUISSE}

Augst : ville.

292) Région XIX : os indéterminé de bovidé ou d'équidé, 2 incisions A-3, B-5, C-1, 2 T, conservée aux deux tiers, L. tête : $12 \mathrm{~mm}$, musée d'Augst, inv. 1989.9.C06305.16. Biblio. : Deschler-Erb, 1998, p. 159 sqq. et pl. 31, n 2041.

293) Schmidmatt, région XVII, E, FK B 08451, vers 350, trouvaille associée avec d'autres épingles, entre autres à tête en pomme de pin: os indéterminé de bovidé ou d'équidé, 2 incisions A-3, B-5, C-1, 2 T, conservée aux deux tiers, L. tête : $16 \mathrm{~mm}$ (fig. $10, \mathrm{n}^{\circ}$ 3), musée d'Augst, inv. 1983.756. Biblio. : Hartmann, 1985, p. 41 et fig. 3, $\mathrm{n}^{\circ} 10$; Deschler-Erb, 1998, p. 159 sqq. et pl. 31, n 2039.

294) Schmidmatt, région XVII, E, FK B 08461, vers 350, trouvaillc associćc avcc d'autrcs ćpinglcs à têtc cn pommc de pin, etc. : os indéterminé de bovidé ou d'équidé, 1 incision A-8, B-5, C-1, 1 T, conservée aux deux tiers, L. tête : $14 \mathrm{~mm}$ (fig. 10, $\mathrm{n}^{\circ} 14$ ), musée d'Augst, inv. 1983.1265. Biblio.: Hartmann, 1985, p. 41 et fig. 3, $\mathrm{n}^{\circ} 12$; Deschler-Erb, 1998, p. 159 sqq. et pl. 31, $\mathrm{n}^{\circ} 2038$.

295) Schmidmatt, région XVII, E, FK B 09014, vers 350, trouvaille associée avec d'autres épingles à tête en pomme de pin, etc. : os indéterminé de bovidé ou d'équidé, 1 incision A-2, B-2, C-1, 2 T, complète, L. tot. : $78 \mathrm{~mm}$, L. tête: $12 \mathrm{~mm}$ (fig. 10, $\mathrm{n}^{\circ} 15$ ), musée d'Augst, inv. 1983.2815. Biblio.: Hartmann, 1985, p. 41 et fig. 3, $\mathrm{n}^{\circ} 11$; Deschler-Erb, 1998, p. 159 sqq. et pl. 31, n 2040.

Kaiseraugst : castrum.

296) Im Rebgarten, trouvaille associée avec des monnaies du milieu du IV's. et une fibule cruciforme type Keller 3B
(Riha 172) : os, 3 incisions A-3, B-2, C-2, 2 T, brisée (conservée aux deux tiers), L. tot. : $60 \mathrm{~mm}$, L. tête : 22 mm, inv. 1989.09.C06305.15. Biblio. : Müller, 1990, p. 96 , fig. $24, \mathrm{n}^{\circ} 29$.

297) Regio XX/X, FK A 8064 (quart nord-est du castrum), fin du III ${ }^{e}$ s.-début du IV ${ }^{e}$ s. (?), trouvée dans une couche hétérogène avec du matériel depuis le $\mathrm{I}^{\mathrm{er}} \mathrm{s}$. jusqu'à la fin du III ${ }^{\mathrm{c}} \mathrm{s}$., éventuellement du début du IV ${ }^{\mathrm{c}} \mathrm{s}$. : os indéterminé de bovidé ou d'équidé, 2 incisions A-2, B-2, C-1, $1 \mathrm{~T}$, conservée aux deux tiers, L. tot. : $74 \mathrm{~mm}$, L. tête : $13 \mathrm{~mm}$ (fig. 10, $\mathrm{n}^{\circ} 11$ ), musée d'Augst, inv. 75.10786. Biblio. : Riha, 1990 , p. 99,161 et pl. 40, 1366 ; DeschlerErb, 1998, p. 174, n 2043 (non illustré).

Pfyn, Städli : Thurgau, castrum.

298) os, 1 incision A-8, C-1, 1 T, brisée. Biblio. : Bürgi, 1983, p. 146-160, fig. 13-14.

299) os, 1 incision A-8, C-1, 1 T, brisée. Biblio. : Bürgi, 1983, p. 146-160, fig. 13-14.

300) os, 1 incision A-8, C-1, 1 T, brisée. Biblio. : Bürgi, 1983, p. 146-160, fig. 13-14.

301) os, 1 incision A-8, C-1, 1 T, complète. Biblio. : Bürgi, 1983, p. 146-160, fig. 13-14.

Windisch, Vindonissa: contexte urbain, comblement d'une cave, daté $\mathrm{du} \mathrm{IV}^{\mathrm{e}} \mathrm{s}$., associée avec d'autres épingles à tête en pomme de pin, etc.

302) os, A-3, C-1, 1 T, pointe brisée. Biblio. : Hartmann, 1980. p. 24, fig. 7 , à gauche.

303) os, A-3, C-1, 1 T, complète. Biblio. : Hartmann, 1980, p. 24, fig. 7 , à droite. 


\section{BIBLIOGRAPHIE}

ALlason-Jones L.

1989: Women in Roman Britain. London, British Museum Publications.

ARBogast R.-M., MÉnIEL P., YVInEC J.-H.

1987 : Une histoire de l'elevage. Les animaux et l'archéologie. Paris, éd. Errance, 104 p.

\section{BARAT Y.}

1993 : "Les céramiques "rugueuses" ou "granuleuses" du Bas-Empire en Île-deFrance ", in Collectif, Trésors de terre. Céramiques et potiers dans l'Île-de-France galloromaine. Catalogue d'exposition, Versailles 1993, Conseil général des Yvelines, Service archéologique départemental, p. 171-177.

BARBIER E.

1962 : « La signification du cortège représenté sur le couvercle du coffret de "Proiecta" ". Cahiers archéologiques, XII, p. 7-33.

BASTIEN P.

1993 : Le busle monétaire des empereurs romains. Wetteren (coll. Numismatique romaine, Essais, recherches et documents, XIX [plus particulièrement, vol. II, p. 573-648 : les impératrices]).

BÉAL J.-C.

1983a : Catalogue des objets de tabletlerie du musée de la Civilisation gallo-romaine de Lyon, Centres d'études romaines et galloromaines de l'université Jean-MoulinLyon III. Paris, Lyon, De Boccard, 421 p., $71 \mathrm{pl}$.

1983b: "Épingles en os à tête féminine à Vienne et dans la région Rhône-Alpes". Bulletin de la Société des amis de Vienne, 78, 4, p. 31-47.

1984 : I es objets en os et en ivoire. Vienne, musées de Vienne, France (coll. Documents, $\left.\mathrm{n}^{\circ} 1\right), 32 \mathrm{p} ., 11 \mathrm{pl}$.

1987 : «Épingles à tête féminine, épingles à tête stylisée ", in Collectir, AutunAuguslodunum, capilale des Éduens. Catalogue d'exposition, Autun, musée Rolin, 16 mars-27 oct. 1985, Ville d'Autun, p. 199-201.

1996 : "Les collections de tabletterie antique du musée Carnavalet ", in Durfoum. J.-F., BÉAl. J.C. (dir.), La tabletterie gallo-romaine et médiévale, une histoire d'os. Catalogue d'art et d'histoire du musée Carnavalet, $\mathrm{XI}$, Paris, Les musées de la ville de Paris, musée Carnavalet, p. 13-17.

BEHRENS G.

1917-1918: "Neue und ältere Funde aus dem Legionskastell Mainz, 4. Kastellbericht ".
Mainzer Zeitschrift, 12/13, p. 21-46.

Bernard J.-L., Celval M.

1994: ZAC Cathédrale Amiens Église (80.021.084 AH), DFS de diagnostic 01/08/1994-30/09/1994. Amiens, Association pour les fouilles archéologiques nationales, Service régional de l'archéologie, ms.

Biehler E., Schlemarre G.

1974 : « Vestiges romains découverts au 4-6 rue du Chanoine-Collin à Metz ". Annuaire de la Société d'histoire et d'archéologie de la Lorraine, LXXIV, p. 9-10.

\section{BILLORET R.}

1974 : « Informations archéologiques, Circonscription de Lorraine: Metz, îlot Saint-Jacques ». Gallia, 32, 2, p. 355-356.

\section{Binet S.}

1994 : « Les objets de tabletterie de l'Antiquité tardive à Châteaubleau (Seine-etMarne) ", in Ouzoullas P., VAN OSSFi. P. (dir.), L'époque romaine tardive en île-deFrance. Paris, Diøecesis Galliarum (Document de travail n ${ }^{\circ}$ ), p. 111-118.

BINSFELD W.

1973 : " Eine Zerstörungsschicht des Jahres 353 in Traben-Trarbach ». Trierer Zeitschrift, 36, p. 119-132.

1984 : "Silberne Büsten », in Colı.fctif, Trier. Kaiserresidenz und Bischofssitz. Die Stadt in spätantiker und frühchristlicher Zeit. Mainz, Philipp von Zabern, p. 118-120.

BISHOP M. C.

1991 : "Soldiers and military equipment in the towns of Roman Britain », in MAXFIELD V. A., Dobson M. J. (dir.), Roman Frontier Studies 1989, Proceedings of the $\mathrm{XV}^{\mathrm{th}}$ International Congress of Roman Frontier Studies. Exeter, University of Exeter Press, p. 21-27.

1996 : Finds from Roman Aldborough, a catalogue of small finds from the Romano-British Town of Isurum Brigatum. Oxbow Monograph, 65.

BrTon R.

1985 : «L'atelier de tablettier du Ve siècle du CD 905 à Nuits-sur-Armançon ". Bulletin de la Sociélé archéologique el historique $d u$ Tonnerrois, 38, p. 9-15.

Blockley K., Blockley M., Blockley P., Frere S., Stow $S$.

1995 : Excavations in the Marlowe Car Park and surrounding areas. Canterbury, Canterbury Archaeological Trust Ltd. (coll. The archaeology of Canterbury, V).
BöHME H. W.

1974: Germanische Grabfunde des 4. bis 5. Jahrhunderts zwischen unterer Elbe und Loire. Münchner Beiträge zur Vor- und

19 Frühgeschichte. München, C. H. Beck, 19, vol. 1 : 384 p.; vol. $2: 147$ pl. et 12 cartes.

Bourgeois A., TUffreau-Libre M.

1981: « Un atelier gallo-romain de taille de l'os à Arras (Pas-de-Calais) ». Latomus, XI., 1, p. 112-120.

BOURGEOIS L. (DIR.)

1999 : Le sanctuaire rural de Bennecourt (Yvelines). Du temple celtique au temple galloromain. Paris, éd. de la Maison des sciences de l'homme (coll. Documents d'archéologie française, 77), $224 \mathrm{p}$.

\section{BRULET R.}

1978 : La fortification de Hauterecenne à Furfooz. Louvain-la-Ncuve, Publications d'histoire de l'art et d'archéologie de l'université catholique de Louvain, XIII, $101 \mathrm{p}$.

BRULET R., VersLyPE L.

1999 : Saint-Pierre de Tournai. L'archéologie d'un monument et d'un quartier. Louvain-laNeuve, Publications d'histoire de l'art et d'archéologie de l'université catholique de Louvain, XCIX.

BucHHOLZ K.

1963 : Die Bildnisse der Kaiserinnen der severischen Zeit nach ihren Frisuren. Frankfurt-amMain.

BÜRGI J.

1983 : " Pfyn-Ad Fines ». Archéologie suisse, 6, p. $146-160$

BuRILLE C.

1996 : Fitude du mobilier de la nécropole de SaintLaurent de Grenoble (IIT-XVIIr s.). Mém. de

126 DEA d'histoire et d'archéologie 6 médiévale, univ. de Provence, Aix-enProvence, ms.

BuSHE-Fox J. P.

1928 : Second report on the excavation of the Roman fort at Richborough, Kent. Oxford, The Society of Antiquiries, London.

CARMElez J.-C.

1982 : « Les objets en os du musée de Bavay (suite) ", in Fouilles et études. Archéologie et pédagogie, lycée de Bavay, 1982, 4, p. 98-117.

CARRIÉ J.-M., RousSeLLe A.

1999 : L'Empire romain en mulation des Sévères à Constantin 192-337. Paris, Seuil (coll. Nouvelle histoire de l'Antiquité, 10), $746 \mathrm{p}$. 
Christophe B., ERTlé R.

1969-1970 : «Les travaux du GEACA au forum de Reims ». Bulletin de la Société archéologique champervise, p. 18-24.

\section{Chuvin P.}

1991 : Chronique des derniers païens. La disparition du paganisme dans l'Empire romain, $d u$ règne de Constantin à celui de Justinien. Paris, Les Belles Lettres/Fayard, $350 \mathrm{p}$.

\section{Colardelle R.}

1999 : Saint-Laurent de Crenoble. De la nécropole gallo-romaine au Monument Historique. Thèse de doctorat, univ. de Provence, Aixen-Provence, ms.

\section{Collectir}

S.d. : Saint-Romain; 8 années de fouilles au lieudit "le Verger "Saint-Romain (Côte-d'Or). Rapport de synthèse 1972-1979 $\mathrm{n}^{\mathrm{c}} 8$ Association de recherches archéologiques de Saint-Romain, ms.

1977 : Objets archéologiques issus des fouilles $d u$ $L A U$ à Tours (1969-1976). Catalogue d'exposition, Tours, 25 mars-15 avril 1977, $48 \mathrm{p}$.

1978: Le cycle de la matière, l'os. Catalogue d'exposition, Dijon, musée archéologique.

1981 (réimpression) : Musée archéologique de Metz. La civilisation gallo-romaine dans la cité des Médiomatrices, $I^{\text {re }}$ partie: monumenis et sanctuaires de l'eau, la vie à la maison, le commerce, les cultes. Metz, Musée de Metz.

- 1983 : Die Römer an Mosel und Saar. Zeugnisse der Römerzeit in Lothringen, in Luxemburg, im Raum Trier und im Saarland, Ausstellung, Bahnhof Rolandseck, 12-28 Sept. 1983. Mainz, Philipp von Zabern, 360 p.

1984a : Ville de Meaux. Catalogues des collections du musée Bossuet. 1. Préhistoire, Protohistoire, Gallo-Romain. Meaux, éd. du musée Bossuet, $191 \mathrm{p}$.

1984b: À l'aube de la France. La Gaule de Constantin à Childéric. Catalogue d'exposition, Paris, musée du Luxembourg, 26 févr.-3 mai 1981, ministère de la Culture et de la Communication, éd. de la Réunion des musées nationaux, $255 \mathrm{p}$.

1984c : Trier. Kaiserresidenz und Bischofssitz. Die Stadt in spätantiker und frühchristlicher Zeit. Mainz, Philipp von Zabern, 373 p.

1989a : Truelles et palissades. 10 ans d'archéologie urbaine, Collégiale Saint-Pierre-le-Puellier. Catalogue d'exposition, Orléans, 17 déc. 1988-12 mars 1989.

1989b : L'Yonne et son passé, 30 ans d'archéologie. Catalogue d'exposition, Comité départemental de la recherche archéologique de l'Yonne, Comité régional de la recherche archéologique de Bourgogne.

1990 : «De la Préhistoire au Moyen Âge », Bulletin du musée Carnavalet, 1-2, p. 47 (ill.).
1994 : Lisieux avant l'an Mil. Essai de reconstitution. Catalogue d'exposition, Lisieux, musées de la ville de Lisieux, 25 juin29 août 1994.

1998 : Profane et sacré en pays meldois. Protohistoire-Gallo-Romain. Meaux, musée Bossuet.

2000 : Le temple gallo-romain de la forêt d'Halatte (Oise). Revue archéologique de Picardie, $\mathrm{n}^{\circ}$ spécial $18,288 \mathrm{p}$.

Cribellier C.

1993 : «Un quartier d'habitat gallo-romain à Pithiviers-le-Vieil (Loiret). Les "Ouches du Bourg” (fouilles 1992-1993) ». Revue archéologique du Loiret, 18, p. 15-98.

Croom A. T.

2000 : Roman Clothing and Fashion. Charleston, éd. Tempus.

Crummy N.

1995 : Colchester Archaeological Report 2: the Roman small finds from excavations in Colchester 1971-1979. Colchester Archaeological Trust Ltd.

Daremberg C., Saglio E. (DIr.)

1887: Dictionnaire des antiquités grecques et romaines d'après les textes et les monuments, t. I, première partie (A-B, sub verbo acus), p. 61-64.

Daveau I., Goustard V.

1995 : Un complexe métallurgique et minier du haut Moyen Age. Habitats gaulois, galloromain et du haut Moyen Âge. Vert-SaintDenis, Ies Fourneaux (77234495-2 AH) Seine-et-Marne, DFS de sauvetage urgent 01.04.1994-31.08.1994. Paris, Saint-Denis, ms.

DEBOISSY J.

1965 : " Le sanctuaire gallo-romain de Vallangoujard ». Bulletin archéologique à Vexin français, 1, p. 71-78.

DE CuPERE B.

2001 : Animals at Ancient Sagalassos. Evidence of the Faunal Remains. Turnhout, Studies in Eastern Mediterranean Archaeology, IV.

DELBRÜCK R.

1933 : Spätantike Kaiserporträts von Constantinus Magnus bis zum Ende des Westreiches. Berlin, Studien zur spätantiken Kunstgeschichte, VIII.

\section{Deschuer-ERB S.}

1998 : Römische Beinartefakte aus Augusta Raurica. Rohmaterial, Technologie, Typologie und Chronologie. Augst, Forschungen in Augst 27, 2 t.

DEYTS S.

1983 : Les bois sculptés des Sources de la Seine. Paris, éd. du CNRS (coll. Suppl. à Gallia, 42), 224 p., $132 \mathrm{pl}$.

1994 : Un peuple de pèlerins. Offrandes en pierre el en bronze des Sources de la Seine. Dijon (coll. Suppl. Revue archéologique de l'Est et du Centre-Est, 13), 208 p.
Dijkman W., ErVynck A.

1998 : Antler, bone, horn, ivory and teeth. The Use of animal skeletal materials in Roman and Early Medieval Maastricht. Maastricht, Archaeologica Mosana, I, 91 p.

\section{DRIESCH A. VON DEN}

1976 : A guide to the measurement of animal bones from archaeological sites. Peabody Museum Bulletins, 1976, 1, $137 \mathrm{p}$.

DUREUIL J.-F.

1994 : « La tabletterie gallo-romaine de la rue de Lutèce " + notices in Paris de l'Antiquité à nos jours. Dix ans d'acquisitions du musée Carnavalet? Bulletin du musée Carnavalet, éd. par Société des Amis du musée Carnavalet, Paris, p. 67-71 et 80-81.

DuREuIL J.-F., BÉAL J.-C.

1996: La tabletterie gallo-romaine et médiévale. Une histoire d'os. Catalogue d'art et d'histoire du musée Carnavalet, XI, Paris, Les musées de la ville de Paris, musée Carnavalet, $123 \mathrm{p}$.

ERTLÉ R.

1968 : «Le travail artisanal de l'os à l'époque gallo-romainc à Reims ". Bulletin de l'Association régionale pour l'éducation et la recherche scientifique, 30.6.1968, p. 13-17.

Evans M. M.

1906 : "Hairdressing of Roman ladies as illustrated on coins ". The Numismatic Chronicle, $4^{\text {th }}$ series, VI, p. 37-65.

FerDitr.re A

1985 : « Informations archéologiques, Circonscription du Centre ». Gallia, 43, 2, p. 297-356.

Ferdière A. (DIR.)

1983 : Un site rural gallo-romain en Beauce: Dambron. S. 1., Publication de la fédération archéologique du Loiret, $106 \mathrm{p}$.

Ferdière A. avec la collab. de Richard G.

1979 : «Fouille de sauvetage du site galloromain de la "Fosse Dieppe" à Dambron (E.-et-L.) ". Revue archéologique du Loiret, 5, p. $31-48$.

Fischer T.

1999 : «Neue Forschungen im spätrömischen Kastell "Haus Bürgel", Stadt Monheim, Kreis Mettman ", in GudkA N. (dir.), Roman Frontier Studies, Proceedings of the XVII $^{\text {th }}$ international Congress of Roman Frontier Studies. Zalau, p. 337-347.

FREMERSDORF $\mathbf{F}$.

1962 : Die römischen Gläser mit aufgelegten Nuppen. Köln, Die Denkmäler des römischen Köln, VII.

FULFORD M. G.

1977 : «Pottery and Britain's foreign trade in the later Roman period ", in Peacock D. S. P. (dir.), Pottery and Early Commerce. Characterization and Trade in Roman and Later Ceramics. London, Academic Press, p. 35-84. 


\section{Gallatad de Sémainvilue $\mathrm{H}$.}

1983 : " Informations archéologiques, Circonscription de Bourgogne ". Gallia, 41, 2, p. 396.

Garnsey P., Saller R.

2001 : L'Empire romain. Économie, société, culture [trad. de l'anglais Franz Regnot]. Paris, I.a Découverte/Poche, 116, 360 p.

Genot G.

1972 : "Sur l'emplacement d'une villa galloromaine à Rigny-la-Salle ". Forum TCF groupe archéologique, 2, p. 51-55.

GEOFFROY-SCHNEITER B.

1998 : Fayoum. Paris, éd. Assouline.

GLLET H.

1928 : "Une tête d'épingle recueillie récemment à Reims, rue du Barbâtre ». Bulletin de la Société archéologique champenoise, 2, p. 40.

\section{Goethert-PolascheK K}

1977 : Kalalog der römischen Gläser des Rheinischen Landesmuseums Trier. Mainz, Trierer Grabungen und Forschungen, IX 352 p. et $81 \mathrm{pl}$.

1983 : "Haarnadeln (acus comatoria) ", in Conl.foctif, Die Römer an Mosel und Saar. Zeugnisse der Römerzeil in Iothringen, in Luxemburg, im Raum Trier und im Saarland, Ausstellung, Bahnhof Rolandseck, 12-28 Sept. 1983. Mainz, von Zabern, p. 277-279.

1984 : " Gläser mit aufgelegten Vertierungen ", in Coll.ectif, Trier. Kaiserresidenz und Bischofssitz. Die Stadt in spälantiker und frühchristlicher Zeit. Mainz, von Zabern, p. 253-264.

Gose E.

1972 : Der gallo-römische Tempelbezirk im Altbachtal $z u$ Trier. Mainz, Trierer Grabungen und Forschungen, VII.

GreEP S.

1982 : Objects of animal bone, antler, ivory and teeth from Roman Britain. D. Phil. thesis, univ. of Cardiff.

GuIllaume P.

1970 : «Les notes de fouilles d'Henri Gillet, 1890-1947 ". Cahiers d'archéologie du nordest, XIII, 24, p. 3-116.

HABEREY W.

1949 : « Ein spātrömisches Frauengrab aus Dorweiler, Kr. Fuskirchen ". Bonner Jahrbücher, 149, p. 82-93.

HAGEN W.

1937 : " Kaiserzeitliche Gagatarbeiten aus dem rheinischen Germanien ". Bonner Jahrbücher, 142, p. 77-144.

Halbardier B., Rausch M.

1969 : "Rapport sommaire des fouilles de Sesselich ". Bulletin de l'Institut archéologique du Luxembourg, XLV, p. 26-45.

HARTMANN M.

1980 : « Castrum Vindonissense. Neues zum spätantiken Kastell von Vindonissa ". Archéologie suisse, 3, p. 23-28.

1985 : « Spätrömisches aus KaiseraugstSchmidmatt ". Archéologie suisse, 1, p. $39-43$.

HEINEN H.

1984 : « Vom Ende des Gallischen Sonderreiches bis zur Usurpation des Magnentius (274-350) ", in Coll.FCTIF, Trier. Kaiserresidenz und Bischofssitz. Die Stadt in spätantiker und frühchristlicher Zeit. Mainz, von Zabern, p. 16-31.

\section{HETTNER F.}

1901 : Drei Tempelbezirke im Trevererlande. Trier, Festschrift zur Feier des 100 Jährigen Bestchens des Gesellschaft für nützliche Forschungen in Trier.

HEURGON J.

1958 : Le trésor de Ténès. Paris, Arts et métiers graphiques.

JACQUES A.

1990 : Les cultes à Arras au Bas-timpire. Catalogue d'exposition, Arras, musée des Beaux-Arts, 26 avr.-17 sept. 1990, Service archéologique de la ville d'Arras.

1993 : « La présence militaire à Arras au BasEmpire ", in VAli.ft F., KaZANSKi M. (dir.), L'armée romaine et les barbares du III au VIT siècle. S. l., mém. de I'AFAM, V, p. $195-208$.

JEDDING-GeSTERLING M. (hrsg.)

1990 : Die Frisur. Eine Kulturgeschichte der Haarmode von der Anlike bis zur Gegenwart. Hamburg, Schwartzkopf.

JEDDING-GeSTERLING M., BRUTSChER G. (DIR.)

S.d. : La coiffure. Une histoire de la mode capillaire de l'Antiquité à nos jours. Neumünster.

JOHNS C., BLAND R.

1994 : " The Hoxne Late Roman Treasure ». Britannia, 25, p. 165-173, pl. IX-XIII.

\section{JoY P. F.}

1989 : «Inventaire des petits objets du site de "La Poulaine" à Épiais-Rhus (Vald'Oise) ". Bulletin archéologique du Vexin français, 22 (1986), p. 77-108.

KASCHNITZ-WEINBERG G. VON

1936 : « Spätrömische Portrăts », in Die Antike, II. Berlin, p. 36 sqq. (réédité dans Kaschnitz-WFinBfrg, G. vON, Ausgewählte Schriften, II, Römische Bildnisse, publié par Kleiner G. vON, HeinTz H. voN, Berlin, 1965, p. 55 sqq.).

\section{Kasparek N., WuHelm J. C. OSB}

2001 : " Für die Schönheil im Jenseits? Zu Fund und Rekonstruktion spätrömischer Kāstchen aus Trier, St. Matthias ». Funde und Ausgrabungen des Rheinischen Landesmuseums Trier, 33, p. 47-62.

\section{KENYON K. M.}

1959: Excavations in Southwark, 1945-1947. Research Paper of the Surrey Archaeological Society, 5.
KERSAUSON K. DE

1996: Catalogue des portraits romains -II- De l'année de la guerre civile (68-69 après J.-C.) à la fin de l'Empire. Paris, musée du Louvre, département des Antiquités grecques, étrusques et romaines.

LAURENT P.

1998 : "Une villa gallo-romaine à EscolivesSainte-Camille ". Bulletin de la Société des sciences historiques el naturelles de l'Yonne, $130^{\mathrm{c}}$ vol., p. $474-479$.

\section{LEgoUX $\mathbf{R}$.}

1998 : « Le cadre chronologique de Picardic : son application aux autres régions $\mathrm{cn}$ vue d'unc chronologie unifiée et son extension vers le romain tardif ", in DEl.ESTRE. X., PÉRIN P. (dir.), Ia datation des structures et des objets du haut Moyen Âge: méthodes et résultats, $\Lambda$ ctes des $\mathrm{XV}^{\text {e journées }}$ de l'AFAM, Rouen, 4-6 févr. 1994. SaintGermain-en-I aye, Association française d'archéologie mérovingienne, p. 137-206.

LOESCHCKE S. (DIR.)

1942 : Der Tempelbezirk im Altbachtale zu Trier, Heft 2. Planausschnitt 2. Die bahndurchschnittenen Tempel und Umgebung. Berlin, Gebr. Mann, 179 p., 33 pl.

\section{L'ORANGE H.-P.}

1984 : Das spätantike Herrscherbild von Diokletian bis zu den Konstantin-Söhnen. Bcrlin.

\section{MASSART C.}

1981 : « Les objets en os ", in BRULET R. (dir.), Braives gallo-romain -I- La zone centrale. Louvain-la-Neuve. Publications d'histoire de l'art et d'archéologie de l'université catholique de Louvain, XXVI, p. 108-109.

\section{MERTEN H.}

2001 : Die Ausgrabungen auf dem Domfreihof (Nordwest-Bereich). Teil 1. Die Funde. Trier, Die Tricrer Domgrabung, 1.

MIKLER H.

1997 : Die römischen Funde aus Bein im Landesmuseum Mainz. Montagnac, Monique Mergoil (coll. Monographies Instrumentum, 1), 173 p., 69 pl., 6 cartes.

\section{MitaRd P.-H.}

$1999^{\circ}$ : "Sur quelques objets peu courants découverts dans les fouilles de l'établissement gallo-romain de "La Garenne" à Vallangoujard (95) ". Bulletin archéologique du Vexin français, 32, p. 73-76.

MOREAU F.

1887: Collection Caranda aux époques préhistorique, gauloise, romaine et franque, album des principaux objets recueillis dans les sépultures de Caranda (Aisne), t. II. Saint-Quentin.

MotTEaU J.

1991 : " Catalogue des objets des fouilles de Tours (1973-1977) ». Recherches sur Tours, vol. 5 . 
MÜLLER U.

1990 : " Ausgrabungen in Kaiseraugst im Jahre 1989 ". Jahresberichte aus Augst und Kaiseraugst, 11, p. 83-98.

\section{MÜLLER-WILLE M.}

1999: Opferkulte der Germanen und Slawen. Stuttgart (Theiss), Sonderheft 1999 der Zcitschrift Archäologic in Deutschland, $102 \mathrm{p}$.

\section{Parlasca K., Seemann H. (Dir.)}

1999 : Augenblicke. Mumienporträts und ägyptische Grabkunst aus römischer Keit. München.

Pelletier A.

1966 : «I.es fouilles du temple de Cybèle à Vienne (Isère). Rapport provisoire ". Revue archéologique, p. 113-150.

\section{PetrT J.-P., Mangin M.}

1994 : Atlas des agglomérations secondaires de la Gaule Belgique et des Germanies. Paris, éd. Errance, $294 \mathrm{p}$.

Petit J.P., Schaur J.

1995 : Bliesbruck Reinheim. Parc archéologique europeen. Paris, Imprimerie nationale (coll. Guides archéologiques de la France), $117 \mathrm{p}$

\section{Petitjean M.}

1960 : "Découverte fortuite de sépultures gallo-romaines à Reims ". Bulletin de la Société archéologique champenoise, 1, p. 18-19.

\section{PILET C.}

1986 : « Informations archéologiques, Circonscription de Basse-Normandie : Baycux - rue Génas-Duhomme - rue Saint-Malo ». Gallia, 44, 2, p. 335-337.

PiRuing R.

1966 : Das römisch-fränkische Gräberfeld von Krefeld-Gellep. Berlin, Germanische Denkmäler der Völkerwanderungszeit, 2, vol. I: 238 p. +19 pl. + 2 dépliants; vol. II. : 157 p. +133 pl.

1974 : Das römisch-fränkische Cräberfeld von Krefeld-Gellep, 1960-1963. Berlin, Germanische Denkmäler der Völkerwanderungszeit, 8, vol. I : 246 p. +9 pl. +1 dépliant; vol. II : 121 p. +159 pl.

Planck D.

2000 : " Denkmalpflege in BadenWürttemberg 1999 ». Denkmalpflege in Baden-Württemberg. Nachrichtenblatt des Landesdenkmalamtes, 29, Jahrgang, 1, p. 2-10.

Plumier J., Dupont J.

1995 : "Fouilles préventives à Rochefort. Deux villae romaines à Ave-et-Auffe et Génimont ", in Pi.umifr J., Corbiau M.-H. (dir.), Troisième journée d'archéologie namuroise, 25 fév. 1995. Namur, ministère de la région wallonne, DGATI.P, p. 83-88.
Prost D.

1981 : « Une cabane d'artisan du IV'e siècle à Escolives-Sainte-Camille ». Bulletin de la Société des sciences historiques et naturelles de l'Yonne, 113, p. 11-51.

1983 : " Le mobilicr en os gallo-romain d'Escolives-Sainte-Camille ». Revue archéologique de l'Est et du Centre-Est, XXXIV, 3-4, p. 263-299.

1989: " La tabletterie ", in Delor J.P., Rol...FY C. (éd.), L'Yonne et son passé, 30 ans d'archéologie. Cataloguc d'cxposition, Comité départemental de la recherche archéologique de l'Yonne, Comité régional de la recherche archéologique de Bourgogne, p. 208-210.

Randoin B., Massat T., Selles H.

1995 : Devant le portail royal. Fouille archéologique du parvis de la cathédrale de Chartres. Catalogue d'exposition, Chartres, Maison de l'archéologie, 8 juil. 1995-28 avr. 1996, Chartres.

RAVETZ A.

1964: "The Fourth Century Inflation and Romano-British coin Finds ». Numismatic Chronicle, p. 201-231.

\section{RIHA E.}

1990 : Der römische Schmuck aus Augst und Kaiseraugst. Bâle, Forschungen in Augst, 10

Roach SMITH C.

1850 : The Antiquities of Richborough. Reculver and Lymne.

\section{RoBerT C.}

1976 : « Une survivance gauloise au IV siècle à La Roche à Lomme à Dourbes (Belgique)". Revue des Rièzes et des Sarts, p. 121-129.

RODET-BeLARBi I.

1990a : « Un atelier du travail de l'os ; la fabrication des épingles ", in Collectir, Melun au temps de Rome. Catalogue d'exposition, 2 nov. 1990-27 janv. 1991, ville de Melun, espace Saint-Jean, p. 46.

1990b : « Le travail de l'os ", in Deschamps S., VAGINAY M. (dir.), Le Mans retrouvé, archéologie et mémoire de la ville. Catalogue d'exposition, L.c Mans, Circonscription des Antiquités préhistoriques et historiques des Pays de la Loire, p. 55.

1999 : « Le travail de l'os et de la corne », in Cilardron-Picault P., Pernot M. (dir.), Un quartier antique d'artisanat métallurgique à Autun, le site du Lycée militaire. Paris, éd. de la Maison des sciences de l'homme (coll. Documents d'archéologie française, 76), p. $250-253$

\section{RoMeUf A.-M.}

2000: Les ex-voto gallo-romains de Chamalières (Puy-de-Dôme) [catalogue des ex-voto sur cédérom par A.-M. Romeuf et
M. DumonteT]. Paris, éd. de la Maison des sciences de l'homme (coll. Documents d'archéologie française, 82), $168 \mathrm{p}$.

RoOsens H.

1962 : Quelques mobiliers funéraires de la fin de l'époque romaine dans le nord de la France. Brugge, Dissertationes archacologicae gandenscs, 7, 42 p. ct 12 pl. h. t.

ROUTIER J.-C.

1990 : TGV Nord Flandre, section 34. Zouafques, Wolphus. Rapport de fouille archéologique, juillet 1990, ms.

Routier J.-C. avec les contributions de Delmatre R., LePETZ S., GoulPEAU L.

À paraître: Le site gallo-romain de Wolphus à Zouafques (Pas-de-Calais). Paris, éd. de la Maison des sciences de l'homme (coll. Documents d'archéologie française).

RoXAN M.

1991 : "Women on the Frontiers ", in MAXFifi.D V. A., DOBSon M. J. (dir.), Roman Frontier Studies 1989, Proceedings of the $\mathrm{XV}^{\mathrm{th}}$ international congress of Roman Frontier Studies. Exeter, Exeter University Press, p. 462-467.

SAINT-BLANQUAT H. DE

1992 : Archéo TGV. $450 \mathrm{~km}$ d'histoire. Tournai, Casterman, $256 \mathrm{p}$.

Santrot M.-H., Santrot J., Tassaux D.

1975 : « Le mobilier d'un puits gallo-romain à Saintes (Charente-Maritime) ». Gallia, 33, 1, p. 117-158.

SÉGUIER J.-M.

1994: "Un ensemble de la fin du III ${ }^{e}$ s. à Saint-Germain-Laxis (Seine-et-Marne) ", in Ouzoulias P., VAN Osser. P. (dir.), l'époque romaine tardive en île-de-France. Paris, Diœcesis Galliarum (coll. Document de travail ${ }^{\circ} 1$ ), p. 145-182.

SÉGUIER J.-M. (DIR.)

1995 : Châtenay-sur-Seine (Seine-et-Marne), "Le Merdat" (77.101.007 AH). Occupations rurales du Haut-Empire, de l'Antiquité tardive et du haut Moyen Âge. Document final de synthèse, Bazoches, Centre départemental d'archéologie de la Bassée, 102 p., ms.

\section{STUTZINGER D.}

1995 : " Römische Haarnadeln mit Frauenbüste ». Bonner Jahrbücher, 195, p. 135-208.

SWIfT E.

2000 : Regionality in Dress Acessories in the Late Roman West, Montagnac, Monique Mergoil (coll. Monographies Instrumentum, 11), $312 \mathrm{p}$.

SYDOW W. voN

1969 : Zur Kunstgeschichte des spätantiken Porträts im 4. Jh. n. Chr: Bonn, Rudolf Habelt Verlag GMBH-Bonn (coll. Antiquitas Reihe 3, Bd. 8). 
THUILLIER F., ROUTIER J.-C.

1998 : "Des imitations de sigillée Drag. 45 (Chenet 328-330) dans la villa galloromaine de Zouafques (Pas-de-Calais) ". Bulletin de la Commission départementale d'histoire et d'archéologie du Pas-de-Calais, Histoire et archéologie du Pas-de-Calais, XVI, p. 3-12.

\section{VAN OSSEL P.}

1992 : Établissemenls ruraux de l'Antiquité tardive dans le nord de la Gaule. Paris, éd. du CNRS (coll. Suppl. à Gallia, 51), 479 p.

1994 : « Inventaire de la céramique sigillée du Bas-Empire conservée au muséc Carnavalet (Paris) ", in Ouzoul.tas P., VAN OSSFI. P. (dir.), L'époque romaine lardive en Île-de-France. Paris, (coll. Document de travail $\mathrm{n}^{\circ} 1$ ), p. 75-101.

Van Ossel P., OuZoulas P.

1987 : « Limetz-Villez 1985-1986. Le site antique et médiéval de la Bosse Marnière ". Connaître les Yvelines, histoire et archéologie, p. 11-15.

VERHAGEN M.

1993 : " Bone and Antler artefacts", in VAN Dierendonck R. M., Hallewas D. P., WAugh K. E. (dir.), The Valkenburg excavations 1985-1988. Introduction and Detail Studies, R.O.B., Valkenburg Project I. Amersfoort, Nederlandse Oudheden, 15, p. $341-418$.

WEGNER M.

1984 : « Die Bildnisse der Frauen und des
Julian ", in L'Orance H.-P. (dir.), Das spätantike Herrscherbild von Diokletian bis zu den Konstantin-Söhnen. Berlin, p. 141-165.

WESSEL $\mathbf{K}$.

1946 : «Römische Frauenfrisuren von der severischen bis zur konstantinischen Zeit ». Jahrbuch des deutschen archäologischen Instituts, 61, p. 62-76.

WHITTAKER C. R.

1989 : Les frontières de l'Empire romain. Paris, Les Belles Lettres (coll. Annales littéraires de l'université de Besançon, 390), 209 p.

WYss M. (DIR.)

1996 : Atlas historique de Saint-Denis. Des origines au XVIII' siècle. Paris, éd. de la Maison des sciences de l'homme (coll. Documents d'archéologic française, 59), $445 \mathrm{p}$. 